\title{
Sobre propagação de ondas eletromagnéticas em meios anisotrópicos
}

\author{
Marcos Paulo de Jesus
}

DISSERTAÇÃO APRESENTADA

$\mathrm{AO}$

INSTITUTO DE MATEMÁTICA E ESTATÍSTICA

DA

UNIVERSIDADE DE SÃO PAULO

PARA

OBTENÇÃO DO TÍTULO

$\mathrm{DE}$

MESTRE EM CIÊNCIAS

Programa: Mestrado em Matemática Aplicada

Orientador: Prof. Dr. Frank Michael Forger

Durante o desenvolvimento deste trabalho

o autor recebeu auxílio financeiro do $\mathrm{CNPq}$

São Paulo, novembro de 2020 



\section{Sobre propagação de ondas eletromagnéticas em meios anisotrópicos}

Esta versão da dissertação contém as correções e alterações sugeridas pela Comissão Julgadora durante a defesa da versão original realizada em 30/11/2020. Uma cópia da versão original encontra-se disponível no Instituto de Matemática e Estatística da Universidade de São Paulo.

Comissão Julgadora:

- Prof. Dr. Frank Michael Forger (Orientador) - IME-USP

- Prof. Dr. Guilherme da Costa Pereira Innocentini - CMCC-UFABC

- Prof. Dr. Pedro Lauridsen Ribeiro - CMCC-UFABC 

A todos que me apoiaram, em particular Jorge e Maria, meus pais. 



\section{Agradecimentos}

Ao meu orientador, Prof. Dr. Frank Michael Forger, não apenas pela oportunidade de trabalhar ao seu lado, mas também pelo apoio, por sua paciência e confiança que nunca faltaram nesses anos todos. Ao programa de Matemática Aplicada do IME-USP, que tornou este trabalho possível. Ao CNPq, pelo financiamento recebido.

À minha família, aos meus amigos, a todos os professores do IME - USP que contribuíram à minha formação e ainda contribuem à formação dos nossos estudantes. 



\section{Resumo}

Esta dissertação trata da propagação de ondas eletromagnéticas em materiais que podem apresentar algum tipo de anisotropia ótica, tais como cristais cujas estruturas não pertencem ao sistema cúbico. Por motivos estéticos sob o ponto de vista matemático, investigamos aqui os efeitos de anisotropias que podem ser tanto elétricas como magnéticas, descritas por um tensor dielétrico $\epsilon$ e um tensor de permeabilidade magnética $\mu$, supondo que ambos sejam simétricos e que comutem, i.e., que sejam simultaneamente diagonalizáveis. Desta forma, generalizamos a abordagem tradicional encontrada na literatura e também na Internet onde, por motivos práticos, consideramse apenas efeitos de anisotropia elétrica mas não de anisotropia magnética, supondo-se que $\epsilon$ seja um tensor mas $\mu$ seja um escalar, i.e., um múltiplo da identidade. Exibimos as equações de ondas satisfeitas pelos campos $\boldsymbol{E}, \boldsymbol{D}$, $\boldsymbol{B}$ e $\boldsymbol{H}$ como exemplos de sistemas de equações diferenciais parciais lineares de segunda ordem que devem ser caracterizados como hiperbólicos sob qualquer ponto de vista pragmático mas claramente fogem do arcabouço da teoria dos operadores normalmente hiperbólicos, que assim se revela demasiadamente estreito para poder responder à pergunta básica: o que é uma definição adequada do conceito de um operador diferencial hiperbólico?

Palavras-chave: Equações de Maxwell em meios anisotrópicos, propagação da luz em materiais birrefringentes. 



\section{Abstract}

This dissertation studies the propagation of electromagnetic waves in materials which can exhibit some kind of optical anisotropy, such as crystals whose structure does not belong to the cubic system. For aesthetical reasons from a mathematical point of view, we investigate here the effects of anisotropies which can be either electric or magnetic, described by a dielectric tensor $\epsilon$ and a magnetic permeability tensor $\mu$, both supposed to be symmetric and to commute with each other, so they are simultaneously diagonalizable. In this sense, we generalize the traditional approach encountered in the literature and also on the Internet where, for practical reasons, only effects of electric anisotropy but not of magnetic anisotropy are considered, supposing that $\epsilon$ is a tensor but $\mu$ is a scalar, i.e., a multiple of the identity. We exhibit the wave equations satisfied by the fields $\boldsymbol{E}, \boldsymbol{D}, \boldsymbol{B}$ and $\boldsymbol{H}$ as examples of systems of linear partial differential equations of second order which should be characterized as hyperbolic from whatever pragmatic point of view but are clearly outside the framework of the theory of normally hyperbolic operators, which in this way reveals itself as too narrow to answer the basic question: what is an adequate definition of the concept of a hyperbolic differential operator?

Keywords: Maxwell's equations in anisotropic media, light propagation in birefringent materials. 



\section{Conteúdo}

Introdução vii

1 Eletromagnetismo no Vácuo 1

1.1 Carga Elétrica . . . . . . . . . . . . . . 2

1.2 Força de Lorentz . . . . . . . . . . . . . . . 5

1.3 Equações de Maxwell . . . . . . . . . . . . . . . . . 6

1.4 Eletrostática . . . . . . . . . . . . . . . . 9

1.5 Magnetostática . . . . . . . . . . . . . 10

1.6 A aproximação estacionária . . . . . . . . . . . . . 11

2 Polarização e Magnetização 13

2.1 Polarização . . . . . . . . . . . . . . . . . . 13

2.2 Magnetização . . . . . . . . . . . . . . . . . 16

3 Eletromagnetismo em Meios $\quad 21$

3.1 Equações de Maxwell . . . . . . . . . . . . . . . . . . 22

3.2 Equações de onda para os campos . . . . . . . . . . . 25

3.3 Equações de onda para os potenciais . . . . . . . . . . 31

3.4 Propagação de ondas . . . . . . . . . . . . . . . 33

3.5 Formulação covariante . . . . . . . . . . . . . 42 



\section{Introdução}

A propagação de ondas eletromagnéticas em meios é um assunto de eletromagnetismo consideravelmente mais complexo do que no vácuo, pois devemse levar em consideração a polarização e a magnetização do meio transportador, que por sua vez são induzidas pelos campos gerados por fontes externas. Fenomenologicamente, conforme descrito por vários textos padrão da área $[3,5,11,12,13,16]$, estes efeitos são captados (a) pela subdivisão da densidade de carga $\rho$ e de corrente $\boldsymbol{j}$ em uma parte "externa" $\rho_{\text {ext }}$ e $\boldsymbol{j}_{\text {ext }}$ e uma outra parte que representa as cargas e correntes induzidas no material, e (b) pela introdução de dois novos campos além do campo elétrico $\boldsymbol{E}$ e da indução magnética $\boldsymbol{B}$, a saber, o deslocamento elétrico $\boldsymbol{D}$ e o campo magnético $\boldsymbol{H}$; então as equações de Maxwell homogêneas são preservadas enquanto que nas equações de Maxwell não homogêneas, substitui-se $\rho$ por $\rho_{\text {ext }}, \boldsymbol{j}$ por $\boldsymbol{j}_{\text {ext }}$, $\boldsymbol{E}$ por $\boldsymbol{D}$ e $\boldsymbol{B}$ por $\boldsymbol{H}$ (a menos de constantes). O sistema é completado pelas equações constitutivas que relacionam $\boldsymbol{D} \operatorname{com} \boldsymbol{E}$ e $\boldsymbol{H} \operatorname{com} \boldsymbol{B}$ e cuja forma explícita depende do material em questão. Junto com essas equações constitutivas, as equações de Maxwell assim modificadas determinam a dinâmica do campo eletromagnético no meio subjacente.

Na sua forma mais simples, as equações constitutivas afirmam que $\boldsymbol{D}$ e $\boldsymbol{E}$, assim como $\boldsymbol{H}$ e $\boldsymbol{B}$, são proporcionais, i.e.,

$$
\boldsymbol{D}=\varepsilon \boldsymbol{E} \quad, \quad \boldsymbol{H}=\mu^{-1} \boldsymbol{B},
$$

onde $\varepsilon$ e $\mu$ são constantes chamadas, respectivamente, de constante dielétrica e de permeabilidade magnética do meio em questão. Uma versão mais geral exige que tal proporcionalidade seja substituída por uma dependência linear, o que significa que ainda prevalece a mesma fórmula; porém, agora $\varepsilon$ e $\mu$ são matrizes. Considerações energéticas [3] implicam que estas matrizes têm que ser simétricas, e para simplificar alguns dos nossos cálculos, suporemos em alguns pontos do texto que elas comutem, para garantir que sejam simultaneamente diagonalizáveis; porém, esta hipótese não é essencial. Como veremos ao longo do trabalho, isso permite descrever os efeitos que um meio anisotrópico exerce sobre a propagação de ondas eletromagnéticas nele. 
Um destes efeitos conhecido há séculos é que a velocidade de propagação de uma onda eletromagnética plana pode depender não apenas da sua direção, mas também de sua polarização, levando ao fenômeno de birrefringência; veja o excelente artigo na Wikipédia sobre este tema [2]. No entanto, não conseguimos encontrar na literatura nenhuma discussão sobre qual seria, nesta situação, a equação de onda generalizada relevante neste caso, e muito menos sobre como o(s) operador(es) de onda generalizado(s) encontrado(s) neste contexto se enquadraria $(\mathrm{m})$ na teoria geral de operadores diferenciais hiperbólicos.

Quanto às possíveis equações constitutivas, vale mencionar que existem muitas outras formas de dependência entre $\boldsymbol{D}$ e $\boldsymbol{E}$ e entre $\boldsymbol{H}$ e $\boldsymbol{B}$ que são mais complexas do que uma simples relação linear. Uma das mais elementares consiste em adicionar ao termo linear um termo constante, o que significa uma polarização ou magnetização do meio mesmo na ausência de campos externos que as induzem, como ocorre em materiais ferroelétricos ou ferromagnéticos. Também pode haver uma dependência verdadeiramente não-linear, o que acontece tipicamente quando os campos externos se tornam muito fortes. E ainda pode ser não-local, o que significa que os valores de $\boldsymbol{D}$ e/ou $\boldsymbol{H}$ num determinado instante $t$ e ponto $\boldsymbol{x}$ no espaço dependem dos valores de $\boldsymbol{E}$ e/ou $\boldsymbol{B}$ em instantes anteriores a $t$ e pontos distintos de $\boldsymbol{x}$. Mesmo mantendo a restrição de linearidade, isso levaria a substituir as simples relações pontuais acima por integrais de convolução do tipo

$$
\begin{gathered}
\boldsymbol{D}(t, \boldsymbol{x})=\int d t^{\prime} d^{3} x^{\prime} \varepsilon\left(t-t^{\prime}, \boldsymbol{x}-\boldsymbol{x}^{\prime}\right) \boldsymbol{E}\left(t^{\prime}, \boldsymbol{x}^{\prime}\right), \\
\boldsymbol{H}(t, \boldsymbol{x})=\int d t^{\prime} d^{3} x^{\prime} \mu^{-1}\left(t-t^{\prime}, \boldsymbol{x}-\boldsymbol{x}^{\prime}\right) \boldsymbol{B}\left(t^{\prime}, \boldsymbol{x}^{\prime}\right),
\end{gathered}
$$

onde o princípio de causalidade exige que o suporte de $\varepsilon$ e $\mu^{-1}$ seja contido no cone de luz passado; então após transformação de Fourier, $\varepsilon$ e $\mu^{-1}$ se tornam funções de $\omega$ e $\boldsymbol{k}$ tais que $\boldsymbol{D}(\omega, \boldsymbol{k})=\varepsilon(\omega, \boldsymbol{k}) \boldsymbol{E}(\omega, \boldsymbol{k})$ e $\boldsymbol{H}(\omega, \boldsymbol{k})=\mu^{-1}(\omega, \boldsymbol{k}) \boldsymbol{B}(\omega, \boldsymbol{k}){ }^{1} \quad$ Em particular, a situação onde $\varepsilon \mathrm{e} / \mathrm{ou} \mu$ dependem da frequência é conhecido como dispersão, a qual do ponto de vista físico é inevitável, mesmo em modelos microscópicos simples [3, Cap. 2.3]. No presente trabalho, porém, não consideraremos nenhum destes cenários mais gerais.

\footnotetext{
${ }^{1} \mathrm{O}$ caso considerado acima é recuperado quando $\varepsilon$ e $\mu$, como funções do tempo e do espaço, são proporcionais à função delta de Dirac e portanto, como funções da frequência e vetor de onda, são constantes.
} 
Do ponto de vista matemático, o trabalho procura dar uma contribuição para orientar a busca por uma resposta a uma questão básica da área de equações diferenciais parciais que por enquanto permanece em aberto: o que seria uma "boa" definição do conceito de um operador diferencial hiperbólico?

Para explicar melhor qual é o problema, vamos adotar o arcabouço da análise global. Suponha que $D: \Gamma(E) \longrightarrow \Gamma(E)$ é um operador diferencial linear, digamos de ordem $r$, agindo nas seções de um determinado fibrado vetorial $E$ sobre uma determinada variedade base $M$. Denotemos por $\sigma_{D}$ o seu símbolo principal, que é uma função $\sigma_{D}: T^{*} M \longrightarrow L(E)$ sobre o fibrado cotangente $T^{*} M$ de $M$ a valores no fibrado vetorial $L(E)$ de transformações lineares de $E$ (isto significa que, em cada ponto $x$ de $M$, a fibra de $L(E)$ em $x$ é o espaço - e de fato, a álgebra - das transformações lineares sobre a fibra de $E$ em $\left.x: L(E)_{x}=L\left(E_{x}\right)\right)$ de modo que, por construção, em cada ponto $x$ de $M, \sigma_{D}(x,$.$) é um polinômio homogêneo de grau r$ sobre o espaço cotangente $T_{x}^{*} M$ a valores no espaço $L(E)_{x}=L\left(E_{x}\right)$ das "matrizes" sobre os "vetores" do "espaço interno" $E_{x}$. Para uso posterior, mencionamos desde já que em muitas situações de interesse, o fibrado vetorial $E$, que pode ser real ou complexo, virá munido de uma métrica nas fibras $\langle.,$. proporcionando, em cada ponto $x$ de $M$, um produto escalar $\langle., .\rangle_{x}$ (i.e., uma forma bilinear simétrica no caso real ou uma forma sesquilinear hermiteana no caso complexo) sobre $E_{x}$ que é não-degenerado, mas em geral não será positivo definido, tal que $\sigma_{D}$ é simétrico, ${ }^{2}$ i.e., $\sigma_{D}(x, \xi)$ é simétrico para todo $x \in M$ e todo $\xi \in T_{x}^{*} M$,

$$
\left\langle\sigma_{D}(x, \xi) u, v\right\rangle_{x}=\left\langle u, \sigma_{D}(x, \xi) v\right\rangle_{x} .
$$

Mais explicitamente, em termos de coordenadas locais $x^{\mu}$ para $M$ e uma trivialização local de $E$ por uma família de seções locais $e_{a}$, temos que para uma seção local qualquer $\psi$ representada na forma $\psi=\psi^{a} e_{a}$ e com a seção local $D \psi$ representada na forma $D \psi=D \psi^{a} e_{a}$ (onde $\left.D \psi^{a} \equiv(D \psi)^{a}\right)$,

$$
D \psi^{a}(x)=\sum_{|\alpha| \leqslant r}\left(a_{\alpha}\right)_{b}^{a}(x) \partial_{\alpha} \psi^{b}(x),
$$

e então, para $\xi=\xi_{\mu} d x^{\mu}(x) \in T_{x}^{*} M$ e $u=u^{a} e_{a}(x) \in E_{x}$,

$$
\left(\sigma_{D}(x, \xi) u\right)^{a}=\sum_{|\alpha|=r}\left(a_{\alpha}\right)_{b}^{a}(x) \xi^{\alpha} u^{b}
$$

\footnotetext{
${ }^{2}$ Aqui, seguimos o abuso de linguagem tradicionalmente adotado na teoria dos operadores em espaços de Hilbert de falar em operadores simétricos mesmo quando, no caso complexo, estamos na verdade lidando com operadores hermiteanos, ou autoadjuntos, e isso mesmo em dimensão finita, ou seja, para matrizes. Além disso, frequentemente omitimos o prefixo "pseudo" que, a rigor, deveria ser empregado cada vez que estamos nos referindo a um produto escalar que não é positivo definido.
} 
Além disso, se $\langle u, v\rangle_{x}=h_{a b}(x) u^{a} v^{b}$ (no caso real) ou $\langle u, v\rangle_{x}=h_{a b}(x) \bar{u}^{a} v^{b}$ (no caso complexo), e se definirmos $\left(a_{\alpha}\right)_{a b}(x)=h_{a c}(x)\left(a_{\alpha}\right)_{b}^{c}(x)$, então $\sigma_{D}$ ser simétrico significa que, para todo multi-índice $\alpha$ de grau máximo $(|\alpha|=r)$, vale $\left(a_{\alpha}\right)_{b a}=\left(a_{\alpha}\right)_{a b}$ (no caso real) ou $\left(a_{\alpha}\right)_{b a}=\left(\bar{a}_{\alpha}\right)_{a b}$ (no caso complexo).

Agora, é bem conhecido que $D$ é chamado de elíptico se, para todo ponto $x$ de $M$ e todo vetor cotangente não nulo $p \in T_{x}^{*} M \backslash\{0\}, \sigma_{D}(x, p)$ é nãosingular (i.e., tem determinante $\neq 0$ ) [4]. Esta é uma "boa" definição em todos os sentidos: independente da escolha de coordenadas locais na variedade base $M$ ou de trivializações locais do fibrado vetorial $E$, invariante sob perturbações do operador por termos de ordem inferior (pois depende apenas do seu símbolo principal), suficientemente forte para provar os teoremas fundamentais da área e ao mesmo tempo suficientemente abrangente para incluir todos os exemplos de operadores elípticos conhecidos da Física Matemática, e por fim, universal no sentido de valer para operadores de qualquer ordem $r$. E por ser "boa" em todos estes sentidos, ela acabou sendo universalmente aceita na comunidade matemática.

Infelizmente, quando passamos para operadores hiperbólicos, a situação é muito menos favorável, pois não se conhece nenhuma definição "boa" em todos estes sentidos. Neste caso, os teoremas fundamentais da área cuja validade deveria ser assegurada são coletivamente conhecidos sob o termo "boa postura do problema de Cauchy" ou, ainda, sob o termo "existência de funções de Green" - motivo pelo qual alguns autores chamam operadores para os quais vale toda essa gama de resultados de "hiperbólicos de Green". Mas é claro que isso é mais uma maneira de abreviar uma meta do que uma definição matemática. Por outro lado, existe todo um zoológico de versões distintas de hiperbolicidade [9], mas infelizmente, cada uma delas sofre de algum defeito fatal. Não queremos nos alongar aqui sobre o tema, mas gostaríamos de mencionar três destas definições que mais se aproximam da meta final: todas elas se referem exclusivamente a propriedades do símbolo principal e portanto satisfazem pelo menos os primeiros dois critérios formulados acima: independência de coordenadas locais e de trivializações locais e invariância sob perturbações do operador por termos de ordem inferior.

A primeira versão é a de um operador fracamente hiperbólico, devida a Gårding [8]: vale para operadores de qualquer ordem, inclui todos os exemplos de operadores hiperbólicos conhecidos da Física Matemática e, segundo um teorema devido a Lax e Mizohata [14], [15], constitui uma condição necessária para a boa postura do problema de Cauchy. Só que, infelizmente, não é suficiente. 
A segunda versão é a de um operador simétrico hiperbólico, devida a Friedrichs [7], com uma importante modificação proposta recentemente por Forger e Vidal [18]: aplica-se apenas a operadores de primeira ordem, mas é suficiente para garantir a boa postura do problema de Cauchy [18]. Porém, a versão original não permite tratar o operador de Dirac sem quebrar a covariância relativística: foi somente através da referida modificação que se tornou possível incluir todos os exemplos de operadores hiperbólicos de primeira ordem conhecidos da Física Matemática.

A terceira versão é a de um operador normalmente hiperbólico, extensamente estudada no livro de Bär, Ginoux e Pfäffle [1]: aplica-se apenas a operadores de segunda ordem, mas é suficiente para garantir a boa postura do problema de Cauchy. Porém, como se evidencia neste trabalho, não inclui todos os exemplos de operadores hiperbólicos de segunda ordem relevantes na Física Matemática, sendo que um exemplo de inegável relevância física tal como a propagação de ondas eletromagnéticas em meios anisotrópicos, colocado em evidência pelo fenômeno de birrefringência, foge deste âmbito.

Sendo assim, a meta seria procurar uma condição sobre o símbolo principal que (a) seja mais forte do que a de hiperbolicidade fraca, (b) no caso $r=1$ se reduza à de hiperbolicidade simétrica (com a referida modificação) e (c) no caso $r=2$ seja mais fraca do que a de hiperbolicidade normal, de modo a abranger a situação tratada neste trabalho. É um desafio que, por enquanto, permanece em aberto. 


\section{Capítulo 1}

\section{Eletromagnetismo no Vácuo}

O eletromagnetismo é o estudo da interação entre partículas eletricamente carregadas. Ao contrário da abordagem empregada na mecânica Newtoniana, esta interação não se dá de forma direta, instantânea e a distância (como na lei universal de atração gravitacional de Newton), mas através de dois mediadores chamados de campo elétrico e campo magnético, que possuem dinâmica própria. Na verdade, essa ideia de mediador foi introduzida na Física por Faraday em torno de 1830, mas originalmente o "campo elétrico" e "campo magnético" foram concebidos como meros artifícios matemáticos para descrever com maior facilidade os fenômenos nesta nova área. No entanto, ao longo dos quase 200 anos que se passaram desde então, o conceito de campo ganhou cada vez mais atributos de uma entidade real, transformando-se em um dos conceitos mais fundamentais da Física moderna.

Com tal abordagem, as leis que governam a referida interação entre partículas eletricamente carregadas se decompõem naturalmente em duas partes: i) a parte que gere a influência de um dado campo elétrico e/ou magnético externo sobre essas partículas, ou mais geralmente sobre uma distribuição de cargas e correntes, e ii) a parte que descreve quais são os campos elétricos e magnéticos gerados por essas partículas, ou mais geralmente por uma dada distribuição de cargas e correntes. A primeira é regida pela força de Lorentz, que inclusive serve para definir o campo elétrico e o campo magnético a partir das forças exercidas sobre "partículas teste", enquanto que a segunda é descrita pelas equações de Maxwell. Em outras palavras, todos os fenômenos eletromagnéticos são descritos pela combinação da força de Lorentz com as equações de Maxwell, sendo que a força de Lorentz diz como os campos afetam cargas e correntes, enquanto que as equações de Maxwell dizem como cargas e correntes geram os campos. Vale ainda mencionar que as equações de Maxwell proporcionaram uma das maiores e mais importantes unificações 
na história da Física, a entre eletricidade e magnetismo (de modo que hoje falamos do "campo eletromagnético"), a qual ainda incluiu, como brinde, a incorporação da ótica como fenômeno eletromagnético.

\subsection{Carga Elétrica}

A fonte de todos os fenômenos eletromagnéticos é a carga elétrica, que é uma propriedade intrínseca fundamental das partículas, tendo um papel duplo: apenas partículas com carga elétrica $\neq 0$ sentem os efeitos de campos eletromagnéticos, e apenas partículas com carga elétrica $\neq 0$ produzem campos eletromagnéticos. (Um pouco mais especificamente, podemos dizer que a eletricidade é associada a cargas por si, enquanto que o magnetismo decorre de cargas em movimento.) Nesse sentido, a carga elétrica é comparável à massa, que age como alvo e também como fonte das forças gravitacionais, mas com a diferença de que a massa é sempre positiva, enquanto que a carga pode ser tanto positiva quanto negativa, correspondendo ao fato que, no caso estático, a força elétrica pode ser tanto repulsiva como atrativa (cargas do mesmo sinal se repelem e cargas de sinal oposto se atraem), enquanto que a força gravitacional é sempre atrativa.

Outra propriedade fundamental da carga elétrica é que é uma quantidade extensiva (o que significa que a carga de um sistema composto de subsistemas é a soma das cargas de cada um deles) e que é conservada: em qualquer processo físico, a carga total antes e depois é a mesma, isto é, cargas não podem ser criadas nem destruídas. Além disso, esta lei de conservação vale localmente, isto é, dentro de qualquer volume: a única forma da carga contida neste volume se alterar é por transporte através da sua superfície. Matematicamente, isto se expressa através de uma equação de continuidade envolvendo um campo escalar chamado de densidade de carga, denotado por $\rho$, e um campo vetorial chamado de densidade de fluxo de carga ou densidade de corrente, denotado por $\boldsymbol{j}$, que é

$$
\frac{\partial \rho}{\partial t}+\nabla \cdot \boldsymbol{j}=0
$$

Tal descrição é inspirada pela hidrodinâmica (dinâmica de fluidos) e baseiase na seguinte interpretação. Em cada instante $t$ e para cada volume $V$, a integral

$$
q_{V}(t)=\int_{V} d^{3} x \rho(t, \boldsymbol{x})
$$




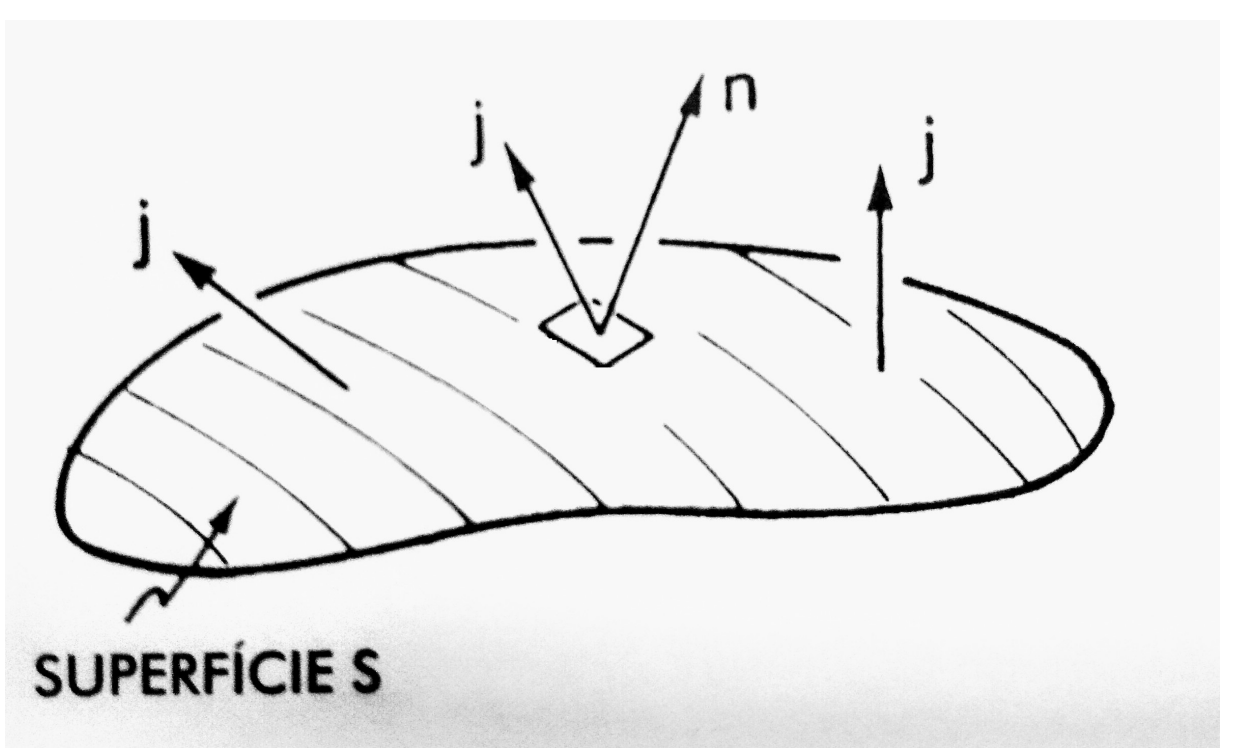

Figura 1.1: A corrente $I_{S}$ através de uma superfície $S$. (Feynman)

representa a carga total contida neste volume neste instante, e para qualquer superfície $S$, a integral

$$
I_{S}(t)=\int_{S} d \boldsymbol{\sigma}(\boldsymbol{x}) \cdot \boldsymbol{j}(t, \boldsymbol{x})=\int_{S} d \sigma(\boldsymbol{x}) \boldsymbol{n}(\boldsymbol{x}) \cdot \boldsymbol{j}(t, \boldsymbol{x})
$$

representa a corrente passando por $S$ no instante $t$. Portanto, para quaisquer dois instantes $t_{0}$ e $t$, com $t>t_{0}$, e qualquer superfície $S$, a integral

$$
\int_{t_{0}}^{t} d t^{\prime} I_{S}\left(t^{\prime}\right)
$$

representa o total da carga transportada através da superfície $S$ durante o intervalo de tempo de $t_{0}$ até $t$ : a corrente é a taxa de fluxo da carga. ${ }^{1}$ Então conservação local da carga elétrica significa que a carga dentro de qualquer volume $V$ só pode mudar por transporte através da sua superfície:

$$
q_{V}(t)-q_{V}\left(t_{0}\right)=-\int_{t_{0}}^{t} d t^{\prime} \int_{\partial V} d \boldsymbol{\sigma}(\boldsymbol{x}) \cdot \boldsymbol{j}\left(t^{\prime}, \boldsymbol{x}\right) .
$$

\footnotetext{
${ }^{1}$ Neste ponto, gostaríamos de acrescentar um comentário sobre a notação empregada aqui e a seguir, referente às regiões no espaço $\mathbb{R}^{3}$ sobre as regiões sobre as quais integramos. Genericamente, denotamos volumes por $V$, superfícies por $S$ e curvas por $\gamma$, sendo que volumes são sempre abertos (com bordo), enquanto que superfícies e curvas podem ser abertas (com bordo) ou fechadas (sem bordo). O bordo de um volume $V$ é uma superfície fechada denotada por $\partial V$, o bordo de uma superfície $S$ aberta é uma curva fechada denotada por $\partial S$ e o bordo de uma curva aberta $\gamma$ é um conjunto $\partial \gamma$ que consiste de dois pontos, a saber, o ponto inicial e o ponto final.
} 


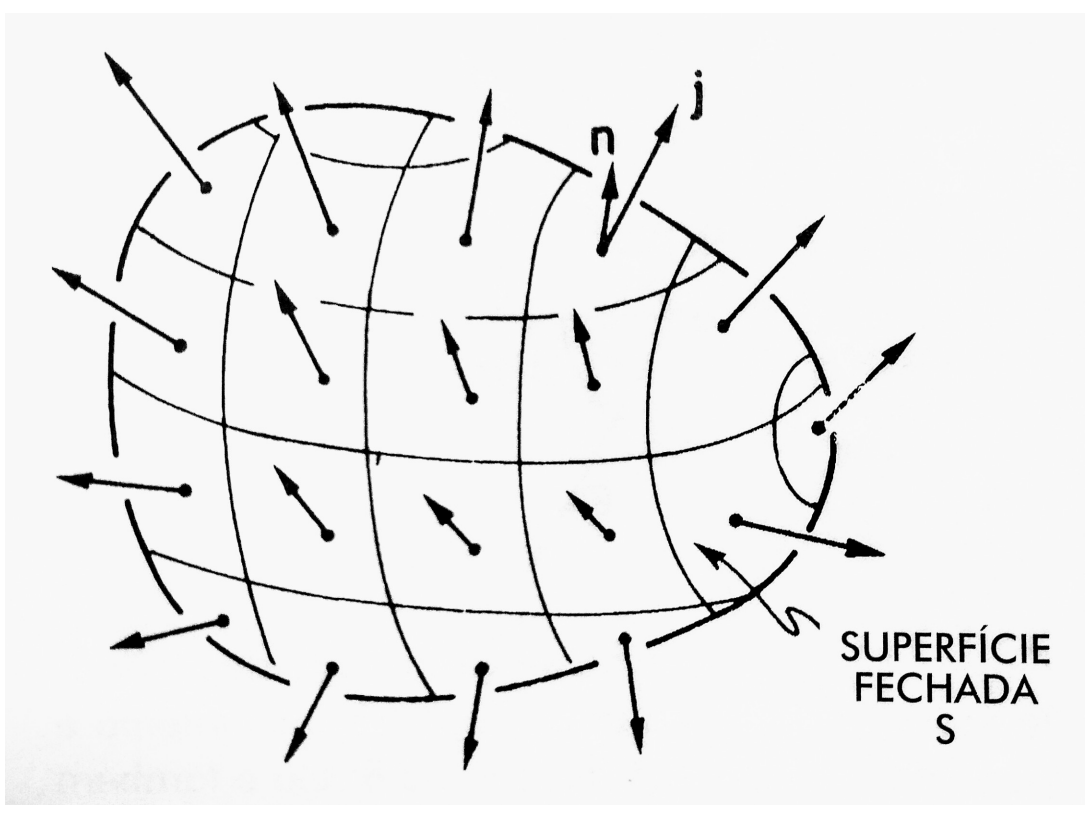

Figura 1.2: A integral da componente normal de $\boldsymbol{j}$ sobre uma superfície fechada igual à taxa de variação da carga interna $q$. (Feynman)

(O sinal negativo segue da convenção de que o campo normal a $\partial V$ deve apontar para fora de $V$, de modo que uma corrente positiva corresponde a uma diminuição da carga dentro do volume.) Diferenciando em relação a $t$, aplicando o teorema de Gauss e usando o fato de que $V$ é arbitrário, chegamos à lei da conservação da carga na sua forma diferencial, equação (1.1).

Finalmente, a carga elétrica é quantizada: usando a carga do próton como referência, todas as cargas de todas as partículas são múltiplos inteiros desta. (Mais exatamente, esta afirmação vale para partículas diretamente observáveis, sendo que quando incluirmos os quarks, ela precisa ser alterada no sentido de que todas as cargas de todas as partículas são múltiplos inteiros de $\frac{1}{3}$ da carga do próton.) Até hoje essa quantização da carga continua sendo um fato empírico para o qual não existe explicação teórica. Uma tentativa para tal explicação, proposta por Dirac, consiste em postular a existência de cargas magnéticas (ou de pelo menos uma partícula no universo com carga magnética), pois neste caso o produto da carga elétrica e da carga magnética percisaria ser quantizado para evitar inconsistências matemáticas nas equações de Maxwell devidamente modificadas. Porém, apesar de buscas intensas, monopolos magnéticos nunca foram observados. 


\subsection{Força de Lorentz}

Como já foi mencionado, a lei da Física que determina a força $\boldsymbol{F}$ que um dado campo elétrico $\boldsymbol{E}$ e um dado campo magnético $\boldsymbol{B}$ exercem sobre uma carga pontual $q$ no instante $t$, quando esta neste instante se encontra na posição $\boldsymbol{x}$ e se move com velocidade $\boldsymbol{v}$, é conhecida como a força de Lorentz:

$$
\boldsymbol{F}=q(\boldsymbol{E}+\boldsymbol{\varkappa} \boldsymbol{v} \times \boldsymbol{B}) .
$$

Aqui, $\varkappa$ é uma constante que depende do sistema de unidades empregado: pode ser 1 ou $1 / c$, onde $c$ é a velocidade da luz no vácuo; comentaremos sobre esta questão no Capítulo 3.

A equação (1.2) tem uma função dupla, pois por um lado ela expressa aspectos das forças eletromagnéticas experimentalmente observados, mas por outro lado também serve como prescrição operacional para definir o campo elétrico $\boldsymbol{E}$ e o campo magnético $\boldsymbol{B}$. Por exemplo, ela afirma que quando separarmos o efeito do ambiente sobre cargas elétricas em propriedades que dependem apenas do ambiente e propriedades que dependem apenas da partícula, as últimas são descritas por uma única quantidade escalar (a carga $q$ ), enquanto que as primeiras são descritas em termos dos dois campos vetoriais $\boldsymbol{E}$ e $\boldsymbol{B}$, que são então determinadas pelas forças que exercem sobre "partículas teste": pequenas cargas elétricas posicionadas em posições diferentes e movendo-se com velocidades diferentes. Por exemplo, a afirmação de que a força eletromagnética depende linearmente da velocidade e em cada instante é ortogonal à direção de movimento da partícula é um fato experimental embutido na lei de força de Lorentz, enquanto que $\boldsymbol{E}$ e $\boldsymbol{B}$ podem ser determinados separadamente comparando as forças sofridas por partículas em movimento com as forças sofridas pelas mesmas partículas em repouso.

Uma versão mais geral da força de Lorentz é obtida quando se considera não apenas uma solitária carga pontual, mas toda uma distribuição de cargas (e de correntes, tendo em vista que cargas em movimento geram correntes). Tal distribuição de cargas e correntes, descrita pela densidade de carga $\rho$ e densidade de corrente $\boldsymbol{j}$ como antes, pode ser tratada como se fosse um "corpo estendido" (ocupando uma região do espaço dada pelo suporte de $\rho$ e de $\boldsymbol{j}$ ), e portante cabe notar que, para corpos estendidos, conforme o princípio de superposição de forças, a força total $\boldsymbol{F}_{V}$ exercida sobre a parte do corpo contida no volume $V$ pode ser escrita como a integral sobre $V$ de uma densidade de força $\boldsymbol{f}$, de modo que uma lei de força que se aplique a corpos estendidos deve ser formulada em termos de uma tal densidade. No caso da força de Lorentz, a generalização da equação (1.2) nestes termos é

$$
\boldsymbol{f}=\rho \boldsymbol{E}+\varkappa \boldsymbol{j} \times \boldsymbol{B} .
$$




\subsection{Equações de Maxwell}

As equações de Maxwell são um conjunto de quatro equações diferenciais parciais de primeira ordem que determinam o campo elétrico $\boldsymbol{E}$ e o campo magnético $\boldsymbol{B}$ gerados por uma dada distribuição de cargas e correntes, relacionando a divergência e o rotacional de $\boldsymbol{E}$ e de $\boldsymbol{B}$ com as suas derivadas em relação ao tempo e com os campos $\rho$ e $\boldsymbol{j}$ que agem como fontes (isto é, constituem os termos que tornam as equações não homogêneas). Explicitamente, são as seguintes:

Lei de Gauss:

$$
\nabla \cdot \boldsymbol{E}=\frac{\rho}{\varepsilon_{0}}
$$

Lei da indução de Faraday:

$$
\nabla \times \boldsymbol{E}=-\varkappa \frac{\partial \boldsymbol{B}}{\partial t}
$$

Ausência de cargas magnéticas:

$$
\boldsymbol{\nabla} \cdot \boldsymbol{B}=0
$$

Lei de Ampère-Maxwell:

$$
\nabla \times \boldsymbol{B}=\varkappa \mu_{0}\left(\boldsymbol{j}+\varepsilon_{0} \frac{\partial \boldsymbol{E}}{\partial t}\right),
$$

Aqui, $\varkappa$ é a mesma constante que antes, enquanto que $\varepsilon_{0}$ e $\mu_{0}$ são constantes conhecidas, respectivamente, como permissividade (elétrica) e permeabilidade (magnética) do vácuo; comentaremos sobre as convenções mais comuns para a fixação destas constantes e sobre sistemas de unidades no Capítulo 3.

Para uma melhor compreensão dos resultados a serem apresentados neste trabalho, parece útil juntar algumas observações bem conhecidas, mas ainda assim importantes, sobre este sistema de equações.

Primeiro, notamos que no caso estático, ou seja, quando todos os campos são independentes do tempo, as equações de Maxwell se dividem em dois subconjuntos disjuntos, a saber:

- As equações da eletrostática:

Lei de Gauss:

$$
\nabla \cdot \boldsymbol{E}=\frac{\rho}{\varepsilon_{0}} .
$$

Conservatividade do campo elétrico:

$$
\boldsymbol{\nabla} \times \boldsymbol{E}=0 .
$$


- As equações da magnetostática:

Ausência de cargas magnéticas:

$$
\boldsymbol{\nabla} \cdot \boldsymbol{B}=0
$$

Lei de Ampère:

$$
\nabla \times \boldsymbol{B}=\varkappa \mu_{0} \boldsymbol{j}
$$

Em outras palavras: eletricidade e magnetismo são fenômenos separados e independentes na estática, sendo que a interação e, no final, a unificação entre eles ocorre quando se tornam dinâmicos.

Segundo, é bastante proveitoso dar uma interpretação física mais concreta para o conteúdo de cada uma dessas equações. Para tanto, torna-se necessário recolocá-las em uma forma integral, o que é feito aplicando os teoremas de Gauss e de Stokes e fazendo uso da seguinte terminologia: para qualquer campo vetorial $\boldsymbol{A}$, a integral de $\boldsymbol{A}$ sobre uma superfície $S$ (aberta ou fechada) é chamada o fluxo de $A$ através de $S$, e a integral de $\boldsymbol{A}$ ao longo de uma curva fechada $\gamma$ é chamada a circulação de $\boldsymbol{A}$ ao longo de $\gamma$. Então tomando a integral de volume das equações (1.4-a) e (1.4-c) (sobre um volume $V$ com bordo $\partial V$ suficientemente regular) e aplicando o teorema de Gauss, constatamos que a primeira delas afirma que

$$
\int_{\partial V} d \boldsymbol{\sigma}(\boldsymbol{x}) \cdot \boldsymbol{E}(t, \boldsymbol{x})=\frac{1}{\varepsilon_{0}} \int_{V} d^{3} x \rho(t, \boldsymbol{x}) \equiv \frac{1}{\varepsilon_{0}} q_{V}(t),
$$

i.e., o fluxo de $\boldsymbol{E}$ através do bordo de qualquer tal volume é (a menos de um fator $\varepsilon_{0}$ que é uma constante universal) igual à carga elétrica contida neste volume, enquanto que a segunda delas afirma que

$$
\int_{\partial V} d \boldsymbol{\sigma}(\boldsymbol{x}) \cdot \boldsymbol{B}(t, \boldsymbol{x})=0 .
$$

i.e., o fluxo de $\boldsymbol{B}$ através do bordo de qualquer tal volume é sempre zero, o que significa que não existem cargas magnéticas. Em termos gráficos, isso significa que as linhas de campo do campo elétrico começam e terminam nas cargas elétricas (com orientação tal que começam em cargas positivas e terminam em cargas negativas), enquanto que as linhas de campo do campo magnético não tem início nem fim: são sempre curvas fechadas. De modo semelhante, tomando a integral de superfície das equações (1.4-b) e (1.4-d) (sobre uma superfície $S$ com bordo $\partial S$ suficientemente regular) e aplicando o teorema de Stokes, constatamos que a primeira delas afirma que

$$
\oint_{\partial S} d \boldsymbol{x} \cdot \boldsymbol{E}(t, \boldsymbol{x})=-\varkappa \frac{d}{d t} \int_{S} d \boldsymbol{\sigma}(\boldsymbol{x}) \cdot \boldsymbol{B}(t, \boldsymbol{x}),
$$


i.e., a circulação de $\boldsymbol{E}$ ao longo do bordo de qualquer tal superfície $S$ é (a menos de um fator $\varkappa$ que é uma constante universal) igual à taxa de variação do fluxo de $\boldsymbol{B}$ através de $S$, mas no sentido de se opor a esta variação ${ }^{2}$, enquanto que a segunda delas afirma que

$$
\oint_{\partial S} d \boldsymbol{x} \cdot \boldsymbol{B}(t, \boldsymbol{x})=\varkappa \mu_{0} \int_{S} d \boldsymbol{\sigma}(\boldsymbol{x}) \cdot \boldsymbol{j}(t, \boldsymbol{x})+\varkappa \mu_{0} \varepsilon_{0} \frac{d}{d t} \int_{S} d \boldsymbol{\sigma}(\boldsymbol{x}) \cdot \boldsymbol{E}(t, \boldsymbol{x}),
$$

i.e., a circulação de $\boldsymbol{B}$ ao longo do bordo de qualquer tal superfície $S$ é igual à soma de dois termos, que (a menos de fatores $\varkappa \mu_{0}$ ou $\varkappa \varepsilon_{0} \mu_{0}$ que são constantes universais) são a corrente $I_{S}$ passando por $S$ e a taxa de variação do fluxo de $\boldsymbol{E}$ através de $S$.

Terceiro, a validade do sistema inteiro só pode ser garantida se a densidade de carga $\rho$ e a densidade de corrente $\boldsymbol{j}$ que aparecem nele satisfazem a equação de continuidade (1.1). Isso segue facilmente combinando a derivada parcial em relação ao tempo da equação (1.4-a) com a divergência da equação (1.4-d). Historicamente, foi este o argumento que levou Maxwell a postular que, para estender a lei de Ampère original (1.8) da magnetostática ao caso de campos com dependência explícita do tempo, se adicione um termo contendo $\partial \boldsymbol{E} / \partial t$. Assim, Maxwell conseguiu eliminar a inconsistência matemática que apareceria caso a lei de Ampère não fosse alterada, pois na sua forma original ela implica que a divergência da corrente deve se anular e portanto a carga contida em qualquer volume teria que ser constante, o que obviamente só pode ser verdade em uma situação estática. Além disso, e quase de brinde, ele previu a existência de ondas eletromagnéticas, abrindo o caminho para umas das maiores unificações de áreas até então distintas da Física: eletricidade, magnetismo e ótica.

Do ponto de vista matemático, a equações de Maxwell são um sistema bem peculiar, pois constituem um sistema de equações diferenciais parciais com vínculos. De fato, impõem vínculos tanto sobre as fontes como sobre as possíveis condições iniciais. O vínculo sobre as fontes é a lei de conservação (1.1), que afirma que as fontes $\rho$ e $\boldsymbol{j}$ não podem ser escolhidas arbitrariamente, mas devem satisfazer sua própria equação diferencial parcial (1.1); caso contrário, as equações de Maxwell não teriam solução alguma. E o vínculo sobre as condições iniciais são as equações (1.4-a) e (1.4-c) que, por não conterem nenhuma derivada temporal, exigem que os campos $\boldsymbol{E}$ e $\boldsymbol{B}$ num instante inicial $t_{0}$ também não podem ser escolhidos arbitrariamente, mas devem satisfazer suas próprias equações diferenciais parciais que restringem (na verdade, fixam) suas divergências.

\footnotetext{
${ }^{2} \mathrm{O}$ fato de que a geração de um campo elétrico com rotação sempre visa se opor à variação do fluxo magnético, expresso pelo sinal -, é conhecida como regra de Lenz.
} 


\subsection{Eletrostática}

As leis básicas da eletrostática são as equações (1.5) e (1.6), requerendo que a densidade de carga $\rho$ independe do tempo. Neste caso, a equação homogênea (1.6) pode ser resolvida pela introdução de um campo escalar $\phi$ chamado de potencial eletrostático, tal que

$$
\boldsymbol{E}=-\nabla \phi
$$

(sendo que o sinal - aqui é meramente convencional), e então a outra equação (1.5) assume a forma

$$
\Delta \phi=-\frac{\rho}{\varepsilon_{0}},
$$

chamada de equação de Poisson. Note que $\phi$ não é unicamente determinado por $\boldsymbol{E}$, mas a liberdade de escolha remanescente é pequena: podemos adicionar a $\phi$ uma constante qualquer sem alterar o conteúdo físico da teoria. ${ }^{3}$ Isso significa que na eletrostática, importam apenas diferenças de potencial, que são integrais de contorno do tipo

$$
\phi\left(\boldsymbol{x}_{2}\right)-\phi\left(\boldsymbol{x}_{1}\right)=-\int_{\gamma} d \boldsymbol{x} \cdot \boldsymbol{E}(\boldsymbol{x}),
$$

onde $\gamma$ é um caminho qualquer (suficientemente regular) com ponto inicial $\boldsymbol{x}_{1}$ e ponto final $\boldsymbol{x}_{2}$, sendo que a integral do lado direito não depende do caminho específico escolhido: tais diferenças de potencial são conhecidas como voltagens.

Do ponto de vista da Física (se bem talvez não da Matemática), a situação mais elementar é quando $\rho$ representa uma carga pontual $q$ situada na origem, ou seja, $\rho(\boldsymbol{x})=q \delta(\boldsymbol{x})$ (onde $\delta$ denota a função delta de Dirac). Neste caso, a solução que possui a mesma invariância sob rotações que a própria equação e que $\delta$, e que se anula no infinito, é o famoso potencial de Coulomb:

$$
\phi_{\text {Coul }}(\boldsymbol{x})=\frac{q}{4 \pi \varepsilon_{0}} \frac{1}{r}
$$

onde $r=|\boldsymbol{x}|$ denota a norma Euclideana de $\boldsymbol{x}$. Tomando o gradiente, obtemos

$$
\boldsymbol{E}_{\text {Coul }}(\boldsymbol{x})=\frac{q}{4 \pi \varepsilon_{0}} \frac{\boldsymbol{r}}{r^{3}}
$$

\footnotetext{
${ }^{3}$ Transformações do tipo $\phi \rightarrow \phi-$ const. são o vestígio na eletrostática do que se chama de transformação de calibre.
} 
Combinando isso com a força de Lorentz, isso leva imediatamente à lei de Coulomb para a força eletrostática $\boldsymbol{F}$ que uma carga pontual $Q$ situada na origem exerce sobre outra carga pontual $q$ situada no ponto $\boldsymbol{x}$ :

$$
\boldsymbol{F}_{\mathrm{Coul}}=\frac{q Q}{4 \pi \varepsilon_{0}} \frac{\boldsymbol{x}}{r^{3}}
$$

\subsection{Magnetostática}

As leis básicas da magnetostática são as equações (1.7) e (1.8), requerendo que a densidade de corrente $\boldsymbol{j}$ independe do tempo. De qualquer forma, a equação homogênea (1.7) pode ser resolvida pela introdução de um campo vetorial $\boldsymbol{A}$ chamado de potencial vetorial, tal que

$$
B=\nabla \times A
$$

e então a outra equação (1.8) assume a forma

$$
\nabla \times(\nabla \times \boldsymbol{A})=\varkappa \mu_{0} \boldsymbol{j} .
$$

Novamente, $\boldsymbol{A}$ não é unicamente determinado por $\boldsymbol{B}$, mas aqui a liberdade de escolha remanescente é maior: podemos adicionar a $\boldsymbol{A}$ o gradiente de um campo escalar $\chi$ qualquer sem alterar o conteúdo físico da teoria. ${ }^{4}$ Isso significa que na magnetostática, importam apenas integrais de contorno do potencial vetorial sobre caminhos fechados, que são integrais de contorno do tipo

$$
\oint_{\gamma} d \boldsymbol{x} \cdot \boldsymbol{A}(\boldsymbol{x})=\int_{S} d \boldsymbol{\sigma}(\boldsymbol{x}) \cdot \boldsymbol{B}(\boldsymbol{x}),
$$

onde $S$ é uma superfície qualquer (suficientemente regular) cujo bordo é $\gamma$, sendo que a integral do lado direito não depende da superfície escolhida.

Podemos fazer uso da indeterminação do potencial vetorial $\boldsymbol{A}$ para impor uma condição adicional, chamada de condição de calibre. Na magnetostática, a condição mais natural deste tipo é o calibre de Coulomb,

$$
\nabla \cdot \boldsymbol{A}=0
$$

(De fato, se inicialmente $\boldsymbol{A}^{\prime}$ é arbitrário, podemos utilizar uma solução $\chi$ da equação diferencial parcial $\Delta \chi=\nabla \cdot \boldsymbol{A}^{\prime}$ para definir um novo potencial

\footnotetext{
${ }^{4}$ Transformações do tipo $\boldsymbol{A} \rightarrow \boldsymbol{A}-\boldsymbol{\nabla} \chi$ são o vestígio na magnetostática do que se chama de transformação de calibre.
} 
vetorial $\boldsymbol{A}=\boldsymbol{A}^{\prime}-\boldsymbol{\nabla} \chi$ que então satisfaz $\boldsymbol{\nabla} \cdot \boldsymbol{A}=0$.) A vantagem desta escolha é que ela reduz a equação não homogênea (1.15) à forma mais simples

$$
\Delta \boldsymbol{A}=-\varkappa \mu_{0} \boldsymbol{j}
$$

que é uma tripla de três equações de Poisson independentes.

Vemos então que a magnetostática e a eletrostática levam ao mesmo problema matemático: o de resolver a equação de Poisson. Isso motivou o desenvolvimento de toda uma área da análise chamada de teoria do potencial. Fisicamente, no entanto, as situações apresentam algumas diferenças notáveis, principalmente porque na eletrostática, o caso mais simples é o de interação entre cargas pontuais, enquanto que na magnetostática, é o de interação entre correntes que, para serem estacionárias, precisam no mínimo ser correntes ao longo de duas curvas fechadas no espaço. Portanto, enquanto que a lei de Coulomb da eletrostática determina a interação de duas cargas $q_{1} \mathrm{e}$ $q_{2}$ situadas em dois pontos $\boldsymbol{x}_{1}$ e $\boldsymbol{x}_{2}$ no espaço, a lei análoga de Biot-Savart da magnetostática especifica a interação de duas correntes $I_{1}$ e $I_{2}$ localizadas em duas curvas fechadas $\gamma_{1}$ e $\gamma_{2}$ no espaço e assim envolve uma integral dupla de contorno sobre $\gamma_{1}$ e $\gamma_{2}$ : omitiremos essa fórmula porque não precisaremos dela neste trabalho.

\subsection{A aproximação estacionária}

Tanto a eletrostática como a magnetostática baseiam-se na hipótese de que a densidade de carga $\rho$ e a densidade de corrente $\boldsymbol{j}$ que geram campos eletrostáticos $\boldsymbol{E}$ e magnetostáticos $\boldsymbol{B}$ não dependem do tempo. No entanto, quando adotarmos um ponto de vista atomístico, considerando toda a matéria como um gás constituído de um imenso número de partículas, visando entender as interações eletromagnéticas como soma de interações entre pares destas partículas, tal hipótese é altamente questionável. Afinal, na eletrostática, cada carga pontual exposta às forças de atração e repulsão eletrostática de todas as outras, mesmo se inicialmente em repouso, logo começará a se colocar em movimento. E na magnetostática, pior ainda pois, por definição, a palavra "corrente" significa "carga em movimento". Portanto, é importante perceber que mesmo assim, é possível estabelecer situações de equilíbrio nas quais as cargas e correntes "efetivas" são representadas por densidades $\rho$ e $\boldsymbol{j}$ que independem do tempo: são estas situações às quais se refere a palavra "estacionária", ao invés de "estática". Por exemplo, em um sólido qualquer, temos um número imenso de núcleos atômicos, normalmente arranjados de forma periódica num cristal, envoltos por seus elétrons, 
dos quais, no caso de um metal (condutor elétrico), uma parte se solta e adquire o status de um gás de elétrons que se move livremente dentro do cristal. Então quando aplicamos um campo elétrico externo a um corpo metálico, este gás de elétrons será deslocado, de modo a criar uma distribuição não homogênea dentro do metal, com excesso de elétrons em certas regiões e deficiência em outras (principalmente perto da superfície do corpo), até chegar a uma situação de equilíbrio representado por uma densidade de carga $\rho$ que não depende do tempo. De modo semelhante, quando aplicamos uma voltagem fixa às extremidades de um fio metálico, o gás de elétrons dentro do fio será colocado em movimento, novamente até chegar a uma situação de equilíbrio com uma corrente representada por uma densidade de corrente $\boldsymbol{j}$ que não depende do tempo e, ainda, possui divergência 0 , pois em cada volume do fio e cada intervalo de tempo, entram tantas cargas quanto saem. É para a descrição de situações como estas que a eletrostática e a magnetostática oferecem ferramentas adequadas e consistentes. 


\section{Capítulo 2}

\section{Polarização e Magnetização}

Neste capítulo, pretendemos abordar a questão de como modificar as equações de Maxwell na presença de matéria, primeiro no caso da eletrostática e da magnetostática (o caso dinâmico será tratado no próximo capítulo). Isso requer discutir os fenômenos de polarização e de magnetização.

\subsection{Polarização}

Quando estudamos o efeito que um campo elétrico externo $\boldsymbol{E}$ exerce sobre a matéria, a primeira distinção que deve ser feita é se o material em questão é um condutor ou um isolante. Do ponto de vista macroscópico, toda a matéria é constituída de um número imenso de átomos ou de moléculas que se organizam de formas diferentes, juntando-se em vários estados de agregação: sólido, líquido, gasoso e plasmático. No caso de um sólido, os átomos ou íons são normalmente arranjados de forma periódica num cristal, envoltos por seus elétrons, sendo que no caso de um condutor, parte destes se solta, transformando-se em um gás de elétrons que se move livremente dentro do cristal, enquanto que no caso de um isolante, tal componente é ausente. No caso de um líquido, também podemos ter líquidos isolantes tais como água destilada e líquidos condutores como soluções salinas, enquanto que gases são sempre isolantes e plasmas são sempre condutores.

De qualquer forma, é claro que num condutor, onde há partículas eletricamente carregadas que podem se movimentar livremente, estas vão reagir à presença de um campo elétrico $\boldsymbol{E}$, sendo aceleradas em direção paralela às linhas de campo. Como esta aceleração é compensada pelos efeitos de colisões 
entre as partículas e, principalmente, com os átomos do cristal $^{1}$, o efeito total é da geração de uma corrente com densidade $\boldsymbol{j}$ proporcional ao campo elétrico, ou seja, $\boldsymbol{j}=\sigma \boldsymbol{E}$ com uma constante $\sigma$ chamada de condutividade do material: é esta relação que é chamada a lei de Ohm.

Passando a considerar isolantes, poderíamos num primeiro momento suspeitar que a presença de um campo elétrico $\boldsymbol{E}$ não teria efeito algum. Entretanto, isso não é verdade. Por exemplo, usando um simples eletroscópio e um capacitor de placas paralelas, Faraday descobriu que a capacitância deste aumentava quando se colocava um isolante entre as placas, e supondo que o isolante preenchesse completamente o espaço entre as placas, aumentava por um fator $\varepsilon_{\mathrm{r}}$ que depende apenas da natureza do material isolante. Tais materiais isolantes também são chamados de dielétricos, e o fator $\varepsilon_{\mathrm{r}}$ é uma propriedade do dielétrico, que recebe o nome de constante dielétrica reduzida, sendo que por convenção, a constante dielétrica reduzida do vácuo vale 1 .

Do ponto de vista microscópico, não é difícil entender por que um material pode ser um dielétrico. Cada átomo possui no seu interior uma carga positiva em seu núcleo, envolta por uma nuvem de elétrons, de carga negativa. Submetido a um campo elétrico, o núcleo será atraído em uma determinada direção e os elétrons na direção oposta, deformando seus orbitais, o que resulta em um deslocamento entre o centro de carga positiva e o centro de carga negativa e assim cria um momento de dipolo elétrico induzido. Além deste, caracterizado por se manifestar apenas na presença de um campo elétrico externo, pode também existir um momento de dipolo elétrico espontâneo, permanente, o qual decorre da geometria das moléculas que compõem o material em questão. Como exemplo bem conhecido, podemos citar a molécula de água, $\mathrm{H}_{2} \mathrm{O}$, que é fortemente polar - ao contrário das moléculas apolares de gás carbônico, $\mathrm{CO}_{2}$, ou de metano, $\mathrm{CH}_{4}$, digamos. No entanto, para entender corretamente como e em quais condições se forma tal momento de dipolo elétrico espontâneo, é imprescindível usar a teoria da ligação química e, mais especificamente, a teoria de repulsão dos pares eletrônicos da camada de valência, que são baseadas inteiramente em princípios da mecânica quântica: a física clássica é completamente incapaz de explicar este fenômeno. Mas independentemente destas questões, podemos afirmar que uma molécula polar, quando submetida a um campo externo, alinhar-se-á com ele de tal modo que seu momento de dipolo se torne paralelo às linhas de campo. Em outras palavras, materiais dielétricos abundam, e pouco importa se a razão de sua polarizabilidade é devido a momentos de

\footnotetext{
${ }^{1}$ Numa análise mais rigorosa usando as leis da mecânica quântica, revela-se que estas colisões ocorrem entre as partículas e os locais de defeitos cristalinos.
} 
dipolo moleculares induzidos ou espontâneos, ou mesmo uma superposição dos dois. (Notamos, de passagem, que em [5] (Capítulo 11), o primeiro tipo é chamado de polarização eletrônica e o segundo de polarização de orientação.)

Então quando expomos um corpo feito por um material dielétrico a um campo elétrico externo, os dipolos elementares nele vão se formar ou alinhar, criando seu próprio campo elétrico, e de modo a se opor ao campo original. De fato, imaginemos um dipolo constituído por duas cargas elétricas muito próximas, do mesmo tamanho mas de sinais opostos. Quando situado sobre uma determinada linha do campo externo, a qual é direcionada de alguma carga positiva para alguma carga negativa (ambas também externas), as duas cargas do dipolo vão se alinhar de tal forma que a negativa delas esteja mais próxima da positiva externa, e vice versa, de modo que a linha de campo induzida pelas duas cargas do dipolo esteja orientada na direção oposta à da linha de campo original.

Essas considerações motivam o seguinte procedimento fenomenológico. Resumimos o efeito gerado por todos os dipolos do material num novo campo vetorial $\boldsymbol{P}$ chamado de polarização (ou mais extensamente, polarização elétrica), que (a menos de um fator $\varepsilon_{0}$ e, mais importante, a menos de um sinal, ambos meramente convencionais) se superpõe ao campo elétrico original $\boldsymbol{E}$, para definir um novo campo $\boldsymbol{D}$ chamado de deslocamento elétrico, conforme

$$
\boldsymbol{D}=\varepsilon_{0} \boldsymbol{E}+\boldsymbol{P} .
$$

Esta decomposição corresponde a uma decomposição em nível das fontes: a densidade de carga total $\rho$ que efetivamente gera o campo elétrico $\boldsymbol{E}$ conforme a lei de Gauss (1.5) é decomposta na soma

$$
\rho=\rho_{\text {pol }}+\rho_{\text {ext }}
$$

de duas contribuições: a densidade de carga de polarização $\rho_{\text {pol }}$ que gera $\boldsymbol{P}$ conforme

$$
\nabla \cdot \boldsymbol{P}=-\rho_{\mathrm{pol}},
$$

e a densidade de carga externa $\rho_{\text {ext }}$ que gera $\boldsymbol{D}$ conforme

$$
\nabla \cdot \boldsymbol{D}=\rho_{\mathrm{ext}} \cdot
$$

No entanto, é importante se atentar ao fato de que não se exige mais que $\boldsymbol{P}$ ou $\boldsymbol{D}$ sejam necessariamente irrotacionais, como é o caso para $\boldsymbol{E}$. Portanto, esta decomposição ainda está incompleta: falta uma relação que determine $\boldsymbol{P}$ em função de $\boldsymbol{E}$.

Antes de discutir esta questão, queremos esclarecer o significado físico da decomposição (2.2). Cada um dos dipolos dentro do material dielétrico gera 
um pequeno desvio da densidade de carga em relação à sua parte original $\rho_{\text {ext }}$, mas estes desvios ocorrem em escala atômica e portanto se compensam em nível macroscópico, exceto na superfície do material, onde se acumulam para formar a contribuição adicional $\rho_{\text {pol }}$ a qual chamamos de densidade de carga de polarização. Por sua natureza, talvez seria até melhor expressá-la como densidade de carga de superfície e não de volume.

Por fim, vamos estabelecer a relação constitutiva entre $\boldsymbol{P}$ e $\boldsymbol{E}$, ou entre $\boldsymbol{D}$ e $\boldsymbol{E}$, que ainda falta para completar o sistema de equações. A relação mais elementar é a de uma simples proporcionalidade,

$$
\boldsymbol{P}=\varepsilon_{0} \chi^{\mathrm{e}} \boldsymbol{E},
$$

que é típica de um meio homogêneo e isotrópico, sendo que a constante $\chi^{\mathrm{e}}$ (que deve satisfazer $\chi^{\mathrm{e}} \geqslant 0$ ) é chamada a susceptibilidade elétrica do material. Então

$$
\boldsymbol{D}=\varepsilon \boldsymbol{E}=\varepsilon_{0} \varepsilon_{\mathrm{r}} \boldsymbol{E}
$$

com

$$
\varepsilon_{\mathrm{r}}=1+\chi^{\mathrm{e}} \text {. }
$$

Em cristais anisotrópicos, encontramos situações nas quais esta relação de proporcionalidade é substituída por uma relação linear mais geral, onde $\chi^{\mathrm{e}}$ e $\varepsilon_{\mathrm{r}}$ são matrizes simétricas, e $\chi^{\mathrm{e}}$ é positiva semidefinida. Mas certamente existem situações muito mais gerais, por exemplo em materiais ferroelétricos onde $\boldsymbol{P}$ pode ser $\neq 0$ mesmo quando $\boldsymbol{E}=0$, ou no caso de campos extremamente fortes quando essa relação funcional deixa de ser linear. Não pretendemos abordar situações deste tipo neste trabalho.

Como ilustrado abaixo. $x_{1}, x_{2}$ e $x_{3}$ e podemos escrever:

\subsection{Magnetização}

De modo análogo ao que foi visto na seção anterior, um campo magnético externo $\boldsymbol{B}$ também exerce efeitos sobre a matéria. Porém, ao contrário do que acontece no caso de um dielétrico, que é sempre atraído pelo campo elétrico, o efeito de um campo magnético pode ser de dois tipos: alguns materiais, chamados de paramagnéticos, são atraídos, enquanto que outros, chamados de diamagnéticos, são repelidos. Por exemplo, alumínio é paramagnético, enquanto que bismuto é diamagnético. (Veja a Figura 2.1.) Ainda existem as substâncias ferromagnéticas, onde a magnetização pode permanecer mesmo quando o campo externo é removido, mas sobre as quais faremos apenas um breve comentário no final das considerações a seguir. 


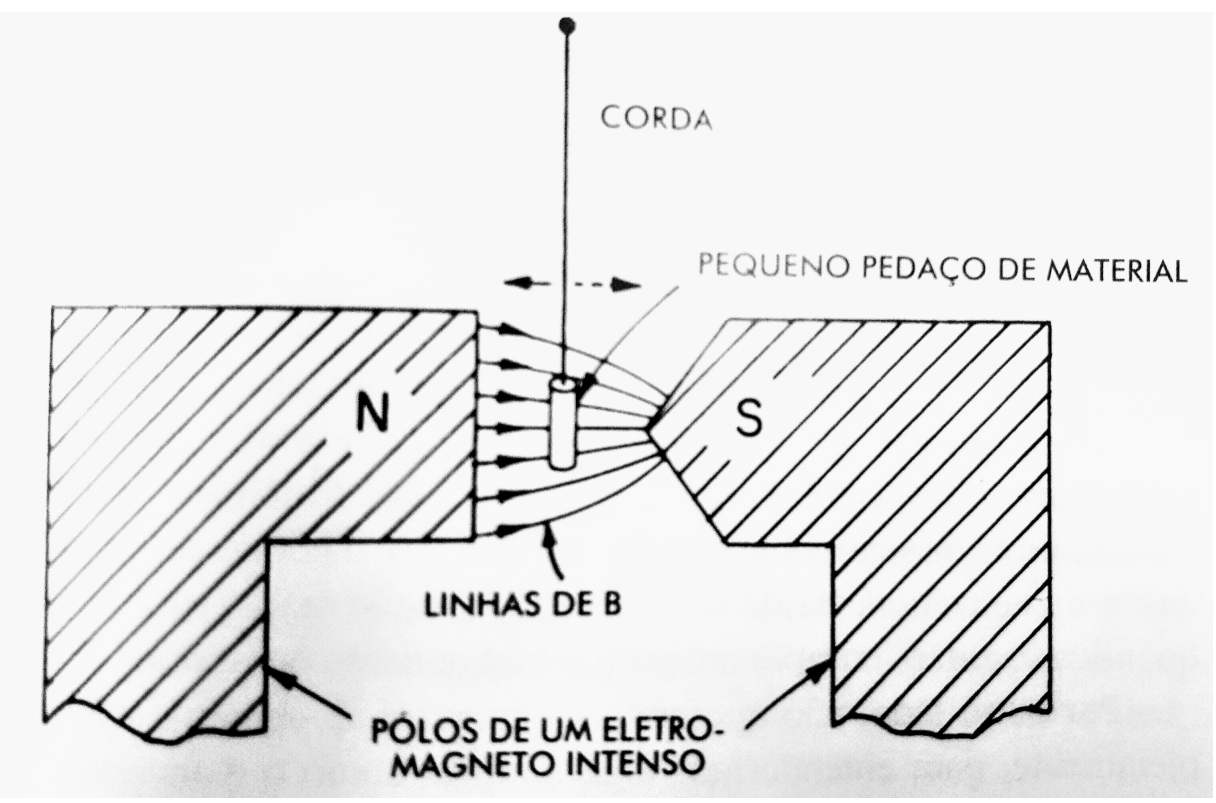

Figura 2.1: Um pequeno cilindro de alumínio é atraído pelo pólo agudo, enquanto que um de bismuto é repelido. (Feynman)

A analogia entre o caso elétrico e o caso magnético ainda vai um pouco além, no sentido de que tanto a polarização como a magnetização podem ser entendidas como resultado da reação de uma distribuição de dipolos (elétricos e magnéticos, respectivamente) no material, e esta pode ser de dois tipos: uma que é induzida pelo próprio campo externo e portanto se anula quando este desaparece, e uma que é pré-existente e permanente. A primeira pode ocorrer em qualquer tipo de material e se deve ao deslocamento dos elétrons dentro de cada átomo devido ao campo externo, o qual cria um momento de dipolo onde antes não havia nenhum, enquanto que a segunda existe apenas em materiais polares, onde os átomos ou as moléculas já possuem um momento de dipolo permanente e então a polarização ou magnetização se dá predominantemente através do alinhamento dos momentos de dipolo já existentes com o campo externo. A diferença é que essas duas contribuições à polarização atuam na mesma direção e portanto se somam, enquanto que as correspondentes contribuições à magnetização atuam em direções opostas, de modo que se torna importante distinguir qual delas predomina: de modo geral, a "parte induzida" age no sentido de reduzir o campo externo e é responsável pelo diamagnetismo em materiais sem momento de dipolo magnético pré-existente, enquanto que a "parte espontânea" age no sentido de reforçar o campo externo e é responsável pelo paramagnetismo em materiais onde há momento de dipolo magnético pré-existente, sendo esta a parte 
dominante nessas circunstâncias.

Novamente, os mecanismos físicos por trás dos fenômenos de diamagnetismo e paramagnetismo, em nível atômico ou molecular, só podem ser compreendidos corretamente quando se usa a mecânica quântica; argumentos clássicos baseados no comportamento de pequenos laços de corrente são, na melhor das hipóteses, guias para alguma intuição (que ainda pode falhar); veja os comentários em [5, Chapter 34]. Qualquer argumentação correta deve se basear no estudo da relação que a mecânica quântica estabelece entre o momento de dipolo magnético e o momento angular, e não abordaremos esta questão aqui.

Ao invés disso, adotamos mais uma vez uma abordagem fenomenológica, na qual resumimos o efeito gerado por todos os dipolos magnéticos do material num novo campo vetorial $\boldsymbol{M}$ chamado de magnetização (ou alternativamente, polarização magnética), que (a menos de um fator $\mu_{0}$ e, mais importante, a menos de um sinal, ambos meramente convencionais) se superpõe ao campo magnético original $\boldsymbol{B}$, para definir um novo campo $\boldsymbol{H}$ também chamado de campo magnético ${ }^{2}$, conforme

$$
\boldsymbol{H}=\mu_{0}^{-1} \boldsymbol{B}-\boldsymbol{M} .
$$

Esta decomposição corresponde a uma decomposição em nível das fontes: a densidade de corrente total $\boldsymbol{j}$ que efetivamente gera o campo magnético $\boldsymbol{B}$ conforme a lei de Ampère (1.8) é decomposta na soma

$$
\boldsymbol{j}=\boldsymbol{j}_{\text {mag }}+\boldsymbol{j}_{\text {ext }}
$$

de duas contribuições: a densidade de corrente de magnetização $\boldsymbol{j}_{\text {mag }}$ que gera $\boldsymbol{M}$ conforme

$$
\nabla \times M=\varkappa j_{\text {mag }},
$$

e a densidade de corrente externa $\boldsymbol{j}_{\text {ext }}$ que gera $\boldsymbol{H}$ conforme

$$
\boldsymbol{\nabla} \times \boldsymbol{H}=\varkappa \boldsymbol{j}_{\text {ext }} .
$$

Novamente, é importante se atentar ao fato de que não se exige mais que $\boldsymbol{M}$ ou $\boldsymbol{H}$ necessariamente tenham divergência nula, como é o caso para $\boldsymbol{B}$.

\footnotetext{
${ }^{2}$ A terminologia empregada aqui requer alguma explicação. Primeiro, é claro que chamar tanto $\boldsymbol{B}$ como $\boldsymbol{H}$ de campo magnético configura um abuso de linguagem. Porém, no caso do eletromagnetismo no vácuo, não há problema, já que os dois campos diferem apenas por um fator $\mu_{0}$ que é uma constante universal. No caso do eletromagnetismo em meios, a terminologia tradicional é de chamar $\boldsymbol{H}$ de campo magnético e $\boldsymbol{B}$ de indução magnética, e seguiremos essa convenção sempre quando for importante distinguir entre os dois, mas mesmo assim, não deixaremos de usar a expressão "campo magnético" também como termo genérico quando tal distinção não for essencial.
} 
Portanto, esta decomposição ainda está incompleta: falta uma relação que determine $\boldsymbol{M}$ em função de $\boldsymbol{B}$.

Essa lacuna é preenchida pela relação constitutiva entre $\boldsymbol{M}$ e $\boldsymbol{B}$, ou entre $\boldsymbol{H}$ e $\boldsymbol{B}$. Mais uma vez, a relação mais elementar é a de uma simples proporcionalidade,

$$
\boldsymbol{M}=\mu_{0}^{-1} \frac{\chi^{\mathrm{m}}}{1+\chi^{\mathrm{m}}} \boldsymbol{B},
$$

que é típica de um meio homogêneo e isotrópico, sendo que a constante $\chi^{\mathrm{m}}$ (que pode ser positiva ou negativa, mas deve satisfazer $\chi^{m}>-1$ ) é chamada a susceptibilidade magnética do material. Então

$$
\boldsymbol{B}=\mu \boldsymbol{H}=\mu_{0} \mu_{\mathrm{r}} \boldsymbol{H},
$$

com

$$
\mu_{\mathrm{r}}=1+\chi^{\mathrm{m}} .
$$

Em cristais anisotrópicos, encontramos situações nas quais esta relação de proporcionalidade é substituída por uma relação linear mais geral, onde $\chi^{\mathrm{m}} \mathrm{e}$ $\mu_{\mathrm{r}}$ são matrizes simétricas, e $\mu_{\mathrm{r}}$ é positiva definida. Mas certamente existem situações muito mais gerais, por exemplo em materiais ferromagnéticos onde $\boldsymbol{H}$ pode ser $\neq 0$ mesmo quando $\boldsymbol{B}=0$ (ou vice versa), ou no caso de campos extremamente fortes quando essa relação funcional deixa de ser linear. Não pretendemos abordar situações deste tipo neste trabalho. 


\section{Capítulo 3}

\section{Eletromagnetismo em Meios}

A nossa meta neste capitulo será estudar primeiro as equações de Maxwell e em seguida a propagação de ondas eletromagnéticas planas em meios. Como foi visto em capítulos anteriores isto requer a introdução de dois novos campos além do campo elétrico $\boldsymbol{E}$ e a indução magnética $\boldsymbol{B}$, a saber, o deslocamento elétrico $\boldsymbol{D}$ e o campo magnético $\boldsymbol{H}$. A nossa hipótese básica será que estes sejam relacionados por equações lineares da forma

$$
\boldsymbol{D}=\varepsilon \boldsymbol{E}, \boldsymbol{B}=\mu \boldsymbol{H},
$$

chamadas de relações constitutivas, onde $\varepsilon$, o tensor dielétrico, e $\mu$, o tensor de permeabilidade, são matrizes simétricas positivas definidas. Portanto, em componentes, temos ${ }^{1}$

$$
\boldsymbol{D}_{i}=\varepsilon_{i j} \boldsymbol{E}_{j}, \boldsymbol{B}_{i}=\mu_{i j} \boldsymbol{H}_{j} .
$$

Como veremos, isso permite incorporar os efeitos de possíveis anisotropias do meio.

No vácuo, $\varepsilon$ e $\mu$ são múltiplos da identidade contendo a constante dielétrica $\varepsilon_{0}$ e a permeabilidade magnética $\mu_{0}$ do vácuo. Assim, é conveniente retirar essas constantes introduzindo o tensor dielétrico reduzido $\varepsilon_{\mathrm{r}}$ e o tensor de permeabilidade reduzido $\mu_{\mathrm{r}}$

$$
\varepsilon_{\mathrm{r}}=\frac{1}{\varepsilon_{0}} \varepsilon, \mu_{\mathrm{r}}=\frac{1}{\mu_{0}} \mu .
$$

Podemos, ainda, expressar $\varepsilon_{r}$ e $\mu_{r}$ em termos dos tensores de susceptibilidade elétrica $\chi^{\mathrm{e}}$ e susceptibilidade magnética $\chi^{\mathrm{m}}$, conforme:

$$
\varepsilon_{\mathrm{r}}=1+\chi^{\mathrm{e}}, \mu_{\mathrm{r}}=1+\chi^{\mathrm{m}} .
$$

\footnotetext{
${ }^{1}$ Usamos sempre a convenção da soma de Einstein.
} 
Por fim, assim como no caso estático, decompomos $\boldsymbol{D}$ e $\boldsymbol{H}$ conforme

$$
\boldsymbol{D}=\varepsilon_{0} \boldsymbol{E}+\boldsymbol{P}, \boldsymbol{H}=\mu_{0}^{-1} \boldsymbol{B}-\boldsymbol{M},
$$

onde $\boldsymbol{P}$ é a polarizaçãa ou mais exatamente a polarização elétrica, e $\boldsymbol{M}$ é a magnetização, também chamada de polarização magnética.

Para obter fórmulas explícitas, suporemos frequentemente que as matrizes que aparecem nas relações constitutivas comutam, para que sejam simultaneamente diagonalizáveis, e então escolheremos uma base de $\mathbb{R}^{3}$, e portanto coordenadas cartesianas $x_{1}, x_{2}, x_{3}$, em que ambas são diagonais,

$$
\varepsilon_{\mathrm{r}}=\left(\begin{array}{ccc}
\varepsilon_{1} & 0 & 0 \\
0 & \varepsilon_{2} & 0 \\
0 & 0 & \varepsilon_{3}
\end{array}\right), \mu_{\mathrm{r}}=\left(\begin{array}{ccc}
\mu_{1} & 0 & 0 \\
0 & \mu_{2} & 0 \\
0 & 0 & \mu_{3}
\end{array}\right) \text {. }
$$

No entanto, para o desenvolvimento do formalismo geral, isso não será imprescindível.

\subsection{Equações de Maxwell}

Começamos escrevendo as equações de Maxwell no vácuo na presença de fontes dadas explicitamente por uma densidade de carga $\rho$ e uma densidade de corrente $\boldsymbol{j}$ :

$$
\begin{gathered}
\nabla \cdot \boldsymbol{E}=\frac{\rho}{\varepsilon_{0}}, \\
\nabla \times \boldsymbol{E}=-\varkappa \frac{\partial \boldsymbol{B}}{\partial t}, \\
\boldsymbol{\nabla} \cdot \boldsymbol{B}=0, \\
\nabla \times \boldsymbol{B}=\varkappa \mu_{0}\left(\boldsymbol{j}+\varepsilon_{0} \frac{\partial \boldsymbol{E}}{\partial t}\right),
\end{gathered}
$$

o que requer que as densidades de carga e de corrente satisfaçam a lei de conservação da carga

$$
\frac{\partial \rho}{\partial t}+\nabla \cdot \boldsymbol{j}=0
$$

Aqui e no restante deste trabalho, seguimos uma convenção adotada em $[6,17]$, a qual garante que as equações se tornem independentes do sistema de unidades usado: isso requer introduzir a constante $\varkappa$ que, dependendo 
do sistema, pode ser 1 ou $1 / c$, onde $c$ é a velocidade da luz no vácuo. Essa mesma constante $\varkappa$ aparece na lei de força de Lorentz: a força $\boldsymbol{F}$ exercida por $\boldsymbol{E}$ e $\boldsymbol{B}$ sobre uma carga pontual $q$ se movendo com velocidade $\boldsymbol{v}$ é

$$
\boldsymbol{F}=q(\boldsymbol{E}+\varkappa \boldsymbol{v} \times \boldsymbol{B}) .
$$

De qualquer modo, as constantes $\varkappa, \varepsilon_{0}, \mu_{0}$ e $c$ satisfazem a seguinte relação:

$$
\varkappa^{2} \varepsilon_{0} \mu_{0}=\frac{1}{c^{2}} \text {. }
$$

Mencionamos que os sistemas de unidades usados na física se dividem em duas grandes classes: os simétricos e os assimétricos. Entre os sistemas simétricos, caracterizados pela escolha $\varkappa=1 / c$, podemos citar o sistema de Gauss $\left(\varepsilon_{0}=1 / 4 \pi, \mu_{0}=4 \pi\right)$ usado na maioria dos tratados sobre a teoria do eletromagnetismo, e o sistema de Heaviside $\left(\varepsilon_{0}=1, \mu_{0}=1\right)$ empregado na física de partículas. Por outro lado, o sistema assimétrico mais importante, onde $\varkappa=1$, é o sistema internacional de medidas e unidades (SI) que hoje predomina em física experimental e engenharia.

Na presença de um material, as equações de Maxwell sofrem modificações substanciais. É verdade que em principio estas equações permanecem em vigor para os campos moleculares (microscópicos) se incluirmos as cargas e correntes de todas as moléculas do material nas fontes. Porém, devido ao enorme número de moléculas envolvidas, tal procedimento é inviável na prática, além de resultar em campos moleculares $\boldsymbol{E}_{\text {mol }}$ e $\boldsymbol{B}_{\text {mol }}$ que apresentam fortes variações espaço-temporais em escalas moleculares e assim, na prática, não são observáveis e nem relevantes. As quantidades de interesse nesse contexto são os campos macroscópicos $\boldsymbol{E}$ e $\boldsymbol{B}$ obtidos tomando-se a média dos seus correspondentes moleculares sobre volumes de ordem $L^{3}$ e intervalos de tempo $T=L / c$ onde a distância $L$ é pequena em termos macroscópicos mas razoavelmente grande quando comparada com dimensões moleculares; tipicamente $L \approx 10^{-7} \mathrm{~m}$.

Porém, como o procedimento de tomar a média é uma operação linear e as equações de Maxwell também são um sistemas de equações diferenciais parciais lineares, segue que os campos macroscópicos satisfazem o mesmo sistema de equações que os campos moleculares, desde que a passagem do nível molecular para o nível macroscópico seja aplicado também às fontes.

Sendo assim, as modificações mencionadas acima, ao contrário do que a maior parte da literatura padrão sobre o assunto sugere, não tem nada a ver com a passagem de campos moleculares para campos macroscópicos. Mas quando um material é colocado sob a influência de uma distribuição de 
cargas e correntes "externas", digamos com densidades $\rho_{\text {ext }}$ e $\boldsymbol{j}_{\text {ext }}$, ocorre um efeito de "feedback", ou retroalimentação, pois o material contém um enorme número de cargas "internas" que vão reagir ao campo eletromagnético gerado pelas fontes externas, sendo que a natureza dessa reação depende fortemente do tipo de material em questão. Por exemplo, em um condutor elétrico, pelo menos parte dessas cargas "internas" pode se mover livremente dentro do material, de modo que o campo externo gera deslocamentos macroscópicos (correntes) conforme a lei de Ohm, enquanto que num isolador elétrico, onde a mobilidade das cargas no material é fortemente limitada, o campo externo gera apenas deslocamentos microscópicos, mas que ainda levam a efeitos macroscópicos observáveis, tais como polarização e magnetização. Agora, em ambos os casos, esses deslocamentos podem ser vistos como produzindo contribuições adicionais $\rho_{\text {mat }}$ à densidade de carga total

$$
\rho=\rho_{\text {ext }}+\rho_{\text {mat }}
$$

e $\boldsymbol{j}_{\text {mat }}$ à densidade de corrente total

$$
\boldsymbol{j}=\boldsymbol{j}_{\mathrm{ext}}+\boldsymbol{j}_{\mathrm{mat}}
$$

que devem ser separadamente conservadas: não apenas a densidade de carga e de corrente total satisfazem a lei de conservação da carga (3.8), mas cada uma das suas duas partes também:

$$
\begin{gathered}
\frac{\partial \rho_{\mathrm{ext}}}{\partial t}+\boldsymbol{\nabla} \cdot \boldsymbol{j}_{\mathrm{ext}}=0 . \\
\frac{\partial \rho_{\mathrm{mat}}}{\partial t}+\boldsymbol{\nabla} \cdot \boldsymbol{j}_{\mathrm{mat}}=0 .
\end{gathered}
$$

De qualquer modo, as cargas e correntes adicionais geram campos eletromagnéticos adicionais, que por sua vez modificam os próprios deslocamentos, etc.. No final deste complexo processo de interação, estabelece-se um equilíbrio que costuma ser descrito fenologicamente, através de uma modificação das equações de Maxwell onde se consideram apenas as cargas e correntes "externas", ou seja, as densidades $\rho_{\text {ext }}$ e $\boldsymbol{j}_{\text {ext }}$, como fontes, complementadas pelas já mencionadas "relações constitutivas".

A forma dessas relações constitutivas varia muito, dependendo da natureza do material. Por exemplo, em condutores elétricos tais como metais, a relação constitutiva principal é a lei de Ohm $\boldsymbol{j}_{\text {mat }}=\sigma \boldsymbol{E}$ onde $\sigma$ é a condutividade do material. Mas existem situações bem mais complexas, tais como na física dos plasmas, onde a necessária adaptação do eletromagnetismo é apenas parte (se bem que parte importante) de toda uma área própria da física conhecida como magnetohidrodinâmica. 
Porém, o nosso objetivo no presente trabalho é outro: analisar a estrutura matemática das equações que governam a propagação de ondas eletromagnéticas em meios anisotrópicos. Tais meios são necessariamente isoladores, pois ondas eletromagnéticas são fortemente atenuadas em condutores elétricos: metais e plasmas não são transparentes!

O resultado final da análise dos fenômenos de polarização e de magnetização, que já foram estudados em capítulos anteriores no caso estático, para as equações de Maxwell é fácil de descrever: preservam-se as equações homogêneas para $\boldsymbol{E}$ e $\boldsymbol{B}$ e modificam-se as equações não homogêneas, mantendo-se apenas as partes externas das fontes e absorvendo-se as partes internas pela substituição de $\varepsilon_{0} \boldsymbol{E}$ por $\boldsymbol{D}$ e de $\mu_{0}^{-1} \boldsymbol{B}$ por $\boldsymbol{H}$. Assim, as equações de Maxwell em meios dielétricos e/ou diamagnéticos ou paramagnéticos assumem a seguinte forma (a ser complementada pelas relações constitutivas $(3.1),(3.2))$ :

$$
\begin{gathered}
\boldsymbol{\nabla} \cdot \boldsymbol{D}=\rho_{\mathrm{ext}}, \\
\nabla \times \boldsymbol{E}=-\varkappa \frac{\partial \boldsymbol{B}}{\partial t}, \\
\boldsymbol{\nabla} \cdot \boldsymbol{B}=0, \\
\boldsymbol{\nabla} \times \boldsymbol{H}=\varkappa\left(\boldsymbol{j}_{\mathrm{ext}}+\frac{\partial \boldsymbol{D}}{\partial t}\right),
\end{gathered}
$$

o que requer que as densidades de carga e de corrente externas satisfaçam a lei de conservação da carga (3.13).

\subsection{Equações de onda para os campos}

Nossa meta nesta seção é deduzir as equações de onda (modificadas) satisfeitas pelos campos $\boldsymbol{E}, \boldsymbol{D}, \boldsymbol{B}, \boldsymbol{H}$ que decorrem das equações de Maxwell (3.15-a)-(3.15-d), em conjunto com as relações constitutivas (3.1), (3.2). Como veremos, vai se tornar muito útil introduzir os seguintes operadores de divergência deformada e do Laplaciano deformado: para qualquer campo vetorial $\boldsymbol{A}$, qualquer campo escalar $\phi$ e qualquer matriz $(3 \times 3)$ simétrica $T$, defina

$$
\boldsymbol{\nabla} \cdot_{T} \boldsymbol{A}=\boldsymbol{\nabla} \cdot(T \boldsymbol{A})=T_{i j} \partial_{i} A_{j},
$$

e

$$
\Delta_{T} \phi=\nabla \cdot{ }_{T} \boldsymbol{\nabla} \phi=T_{i j} \partial_{i} \partial_{j} \phi
$$


sendo que abreviaremos $\boldsymbol{\nabla} \cdot \varepsilon_{\varepsilon_{\mathrm{r}}} \boldsymbol{A}$ para $\boldsymbol{\nabla}{ }_{\varepsilon} \boldsymbol{A}, \boldsymbol{\nabla} \cdot{ }_{\mu_{\mathrm{r}}} \boldsymbol{A}$ para $\boldsymbol{\nabla} \cdot{ }_{\mu} \boldsymbol{A}, \Delta_{\varepsilon_{\mathrm{r}}} \phi$ para $\Delta_{\varepsilon} \phi$ e $\Delta_{\mu_{\mathrm{r}}} \phi$ para $\Delta_{\mu} \phi$. Prosseguimos então essencialmente da mesma forma como no caso tradicional do eletromagnetismo no vácuo.

Primeiro, inserimos a relação $\boldsymbol{B}=\mu \boldsymbol{H}$ na equação (3.15-b) para escrever

$$
\mu^{-1} \nabla \times \boldsymbol{E}=-\varkappa \frac{\partial \boldsymbol{H}}{\partial t}
$$

e aplicando novamente o rotacional, podemos então usar a equação (3.15-d) para obter

$$
\boldsymbol{\nabla} \times\left(\mu^{-1} \boldsymbol{\nabla} \times \boldsymbol{E}\right)=-\varkappa \frac{\partial}{\partial t}(\boldsymbol{\nabla} \times \boldsymbol{H})=-\varkappa^{2} \frac{\partial \boldsymbol{j}_{\mathrm{ext}}}{\partial t}-\varkappa^{2} \frac{\partial^{2} \boldsymbol{D}}{\partial t^{2}},
$$

o que, devido à relação $\boldsymbol{D}=\varepsilon \boldsymbol{E}$, nos dá a seguinte equação para $\boldsymbol{E}$ :

$$
\varepsilon\left(\varkappa^{2} \frac{\partial^{2} \boldsymbol{E}}{\partial t^{2}}\right)+\nabla \times\left(\mu^{-1} \boldsymbol{\nabla} \times \boldsymbol{E}\right)=-\varkappa^{2} \frac{\partial \boldsymbol{j}_{\mathrm{ext}}}{\partial t} .
$$

De forma completamente análoga, inserimos a relação $\boldsymbol{D}=\varepsilon \boldsymbol{E}$ na equação (3.15-d) para escrever

$$
\varepsilon^{-1} \nabla \times \boldsymbol{H}=\varkappa \varepsilon^{-1} \boldsymbol{j}_{\mathrm{ext}}+\varkappa \frac{\partial \boldsymbol{E}}{\partial t},
$$

e aplicando novamente o rotacional, podemos então usar a equação (3.15-b) para obter

$$
\begin{aligned}
\boldsymbol{\nabla} \times\left(\varepsilon^{-1} \boldsymbol{\nabla} \times \boldsymbol{H}\right) & =\varkappa \boldsymbol{\nabla} \times\left(\varepsilon^{-1} \boldsymbol{j}_{\mathrm{ext}}\right)+\varkappa \frac{\partial}{\partial t}(\boldsymbol{\nabla} \times \boldsymbol{E}) \\
& =\varkappa \boldsymbol{\nabla} \times\left(\varepsilon^{-1} \boldsymbol{j}_{\mathrm{ext}}\right)-\varkappa^{2} \frac{\partial^{2} \boldsymbol{B}}{\partial t^{2}},
\end{aligned}
$$

o que, devido à relação $\boldsymbol{B}=\mu \boldsymbol{H}$, nos dá a seguinte equação para $\boldsymbol{H}$ :

$$
\mu\left(\varkappa^{2} \frac{\partial^{2} \boldsymbol{H}}{\partial t^{2}}\right)+\nabla \times\left(\varepsilon^{-1} \boldsymbol{\nabla} \times \boldsymbol{H}\right)=\varkappa \nabla \times\left(\varepsilon^{-1} \boldsymbol{j}_{\text {ext }}\right) .
$$

Aqui, é conveniente passar para os tensores reduzidos e usar a relação (3.10) para eliminar as constantes dimensionais do lado esquerdo das equações (3.18) e (3.19), que então assumem a forma

$$
\varepsilon_{\mathrm{r}}\left(\frac{1}{c^{2}} \frac{\partial^{2} \boldsymbol{E}}{\partial t^{2}}\right)+\nabla \times\left(\mu_{\mathrm{r}}^{-1} \nabla \times \boldsymbol{E}\right)=-\frac{1}{\varepsilon_{0} c^{2}} \frac{\partial \boldsymbol{j}_{\mathrm{ext}}}{\partial t}
$$


$\mathrm{e}$

$$
\mu_{\mathrm{r}}\left(\frac{1}{c^{2}} \frac{\partial^{2} \boldsymbol{H}}{\partial t^{2}}\right)+\nabla \times\left(\varepsilon_{\mathrm{r}}^{-1} \nabla \times \boldsymbol{H}\right)=\kappa \boldsymbol{\nabla} \times\left(\varepsilon_{\mathrm{r}}^{-1} \boldsymbol{j}_{\text {ext }}\right) .
$$

Por fim, acrescentamos um termo que se anula devido às equações de Maxwell (3.15-a) e (3.15-c) para reescrever estas equações (e as equações análogas para $\boldsymbol{B}$ e $\boldsymbol{D})$ na forma final

$$
\begin{gathered}
\frac{1}{c^{2}} \frac{\partial^{2} \boldsymbol{E}}{\partial t^{2}}-\lambda \boldsymbol{\nabla}\left(\boldsymbol{\nabla} \cdot{ }_{\varepsilon} \boldsymbol{E}\right)+\varepsilon_{\mathrm{r}}^{-1} \boldsymbol{\nabla} \times\left(\mu_{\mathrm{r}}^{-1} \boldsymbol{\nabla} \times \boldsymbol{E}\right) \\
=-\frac{1}{\varepsilon_{0} c}\left(\frac{1}{c} \frac{\partial}{\partial t}\left(\varepsilon_{\mathrm{r}}^{-1} \boldsymbol{j}_{\mathrm{ext}}\right)+\lambda c \boldsymbol{\nabla} \rho_{\mathrm{ext}}\right), \\
\frac{1}{c^{2}} \frac{\partial^{2} \boldsymbol{B}}{\partial t^{2}}-\lambda \boldsymbol{\nabla}(\boldsymbol{\nabla} \cdot \boldsymbol{B})+\boldsymbol{\nabla} \times\left(\varepsilon_{\mathrm{r}}^{-1} \boldsymbol{\nabla} \times\left(\mu_{\mathrm{r}}^{-1} \boldsymbol{B}\right)\right) \\
=\varkappa \mu_{0} \boldsymbol{\nabla} \times\left(\varepsilon_{\mathrm{r}}^{-1} \boldsymbol{j}_{\mathrm{ext}}\right), \\
\frac{1}{c^{2}} \frac{\partial^{2} \boldsymbol{D}}{\partial t^{2}}-\lambda \varepsilon_{\mathrm{r}} \boldsymbol{\nabla}(\boldsymbol{\nabla} \cdot \boldsymbol{D})+\boldsymbol{\nabla} \times\left(\mu_{\mathrm{r}}^{-1} \boldsymbol{\nabla} \times\left(\varepsilon_{\mathrm{r}}^{-1} \boldsymbol{D}\right)\right) \\
=-\frac{1}{c}\left(\frac{1}{c} \frac{\partial \boldsymbol{j}_{\mathrm{ext}}}{\partial t}+\lambda c \varepsilon_{\mathrm{r}} \boldsymbol{\nabla} \rho_{\mathrm{ext}}\right), \\
\frac{1}{c^{2}} \frac{\partial^{2} \boldsymbol{H}}{\partial t^{2}}-\lambda \boldsymbol{\nabla}\left(\boldsymbol{\nabla} \cdot{ }_{\mu} \boldsymbol{H}\right)+\mu_{\mathrm{r}}^{-1} \boldsymbol{\nabla} \times\left(\varepsilon_{\mathrm{r}}^{-1} \boldsymbol{\nabla} \times \boldsymbol{H}\right) \\
=\varkappa \mu_{\mathrm{r}}^{-1} \boldsymbol{\nabla} \times\left(\varepsilon_{\mathrm{r}}^{-1} \boldsymbol{j}_{\mathrm{ext}}\right),
\end{gathered}
$$

onde $\lambda$ é um parâmetro de Lagrange, cujo valor deve ser irrelevante para qualquer quantidade observável, para incorporar as equações de Maxwell (3.15-a) e (3.15-c) como vínculos

Agora, mesmo no caso do eletromagnetismo no vácuo, onde vale $\varepsilon_{\mathrm{r}}=1 \mathrm{e}$ $\mu_{\mathrm{r}}=1$, completar a derivação das equações de onda pertinentes ainda requer a eliminação do rotacional duplo, através da identidade

$$
\nabla \times(\nabla \times A)=\nabla(\nabla \cdot A)-\Delta A
$$

que vale para qualquer campo vetorial $\boldsymbol{A}$. A relação análoga para o duplo rotacional "torcido" com o qual estamos lidando aqui é a fórmula

$$
\left.\boldsymbol{\nabla} \times T^{-1}(\boldsymbol{\nabla} \times \boldsymbol{A})=\frac{1}{\operatorname{det} T} T\left(\boldsymbol{\nabla}\left(\boldsymbol{\nabla} \cdot_{T} \boldsymbol{A}\right)\right)-\Delta_{T} \boldsymbol{A}\right),
$$

que vale para qualquer campo vetorial $\boldsymbol{A}$ e qualquer matriz $(3 \times 3)$ invertível e simétrica $T$. Como jamais vimos esta fórmula em lugar algum na literatura, vamos dar uma demonstração, que é baseada na fórmula dos cofatores para 
os elementos da inversa de uma matriz invertível $T$, a qual afirma que se denotarmos por $\check{T}_{j i}$ a matriz obtida a partir de $T$ por eliminação da $j$-ésima linha e da $i$-ésima coluna, e se definirmos $\operatorname{cof}(T)_{j i}=(-1)^{i+j} \operatorname{det} \check{T}_{j i}$, então vale $\left(T^{-1}\right)_{i j}=\operatorname{cof}(T)_{i j} / \operatorname{det} T[10$, Cap. $4, \S 5]$. Para matrizes $(3 \times 3)$, podemos reescrever esta afirmação usando o tensor de volume padrão $\epsilon\left(\epsilon_{i j k}=+1(-1)\right.$ se $(i, j, k)$ é uma permutação cíclica (anticíclica) de $(1,2,3)$ e $\epsilon_{i j k}=0$ se $(i, j, k)$ não é uma permutação de $(1,2,3))$ e o tensor de Kronecker padrão $\delta$ $\left(\delta_{i j}=1\right.$ se $i=j$ e $\delta_{i j}=0$ se $\left.i \neq j\right)$, os quais satisfazem as seguintes regras básicas:

$$
\begin{gathered}
\epsilon_{i j k} \epsilon_{k l m}=\delta_{i l} \delta_{j m}-\delta_{i m} \delta_{j l} . \\
\frac{1}{2} \epsilon_{i k l} \epsilon_{j k l}=\delta_{i j} .
\end{gathered}
$$

Note que, como $(\boldsymbol{\nabla} \times \boldsymbol{A})_{i}=\epsilon_{i j k} \partial_{j} A_{k}$, a fórmula (3.24) segue imediatamente da fórmula (3.26):

$$
\begin{aligned}
& (\boldsymbol{\nabla} \times(\boldsymbol{\nabla} \times \boldsymbol{A}))_{i}=\epsilon_{i j k} \partial_{j}(\boldsymbol{\nabla} \times \boldsymbol{A})_{k}=\epsilon_{i j k} \epsilon_{k l m} \partial_{j} \partial_{l} A_{m} \\
& =\left(\delta_{i l} \delta_{j m}-\delta_{i m} \delta_{j l}\right) \partial_{j} \partial_{l} A_{m}=\partial_{i} \partial_{j} A_{j}-\partial_{j} \partial_{j} A_{i} \\
& =(\boldsymbol{\nabla}(\boldsymbol{\nabla} \cdot \boldsymbol{A})-\boldsymbol{\Delta} \boldsymbol{A})_{i} .
\end{aligned}
$$

Agora podemos reescrever a fórmula dos cofatores na seguinte forma,

$$
\left(T^{-1}\right)_{i j}=\frac{1}{\operatorname{det} T} \frac{1}{2} \epsilon_{i k l} \epsilon_{j p q} T_{p k} T_{q l}
$$

a qual também pode ser escrita na forma

$$
\epsilon_{i j p}\left(T^{-1}\right)_{p q} \epsilon_{q k l}=\frac{1}{\operatorname{det} T}\left(T_{k i} T_{l j}-T_{k j} T_{l i}\right) .
$$

A prova mais simples da equação (3.28) baseia-se na fórmula

$$
\epsilon_{r p q} T_{p k} T_{q l} T_{r j}=(\operatorname{det} T) \epsilon_{k l j}
$$

que é uma consequência imediata da observação de que o lado esquerdo é um tensor totalmente antissimétrico em $(k, l, j)$, combinada com a definição do determinante. Multiplicando com $\frac{1}{2} \epsilon_{i k l}$, somando sobre $k$ e $l$ e usando a equação (3.27), segue que

$$
\frac{1}{2} \epsilon_{i k l} \epsilon_{r p q} T_{p k} T_{q l} T_{r j}=(\operatorname{det} T) \delta_{i j},
$$


o que já prova a afirmação (3.28). Com isso, também podemos facilmente provar a equação (3.29),

$$
\begin{aligned}
\epsilon_{i j p}\left(T^{-1}\right)_{p q} \epsilon_{q k l} & =\frac{1}{\operatorname{det} T} \frac{1}{2} \epsilon_{i j p} \epsilon_{p m n} \epsilon_{q r s} \epsilon_{q k l} T_{r m} T_{s n} \\
& =\frac{1}{\operatorname{det} T} \frac{1}{2}\left(\delta_{i m} \delta_{j n}-\delta_{i n} \delta_{j m}\right)\left(\delta_{r k} \delta_{s l}-\delta_{r l} \delta_{s k}\right) T_{r m} T_{s n} \\
& =\frac{1}{\operatorname{det} T}\left(T_{k i} T_{l j}-T_{l i} T_{k j}\right),
\end{aligned}
$$

e a partir dessa a equação (3.25), no caso em que a matriz $T$ for simétrica,

$$
\begin{aligned}
\left(\boldsymbol{\nabla} \times T^{-1}(\boldsymbol{\nabla}\right. & \times \boldsymbol{A}))_{i}=\epsilon_{i j k} \partial_{j}\left(\left(T^{-1}\right)_{k l}(\boldsymbol{\nabla} \times \boldsymbol{A})_{l}\right) \\
& =\epsilon_{i j k}\left(T^{-1}\right)_{k l} \epsilon_{l r s} \partial_{j} \partial_{r} A_{s} \\
& =\frac{1}{\operatorname{det} T}\left(T_{r i} T_{s j}-T_{s i} T_{r j}\right) \partial_{j} \partial_{r} A_{s} \\
& =\frac{1}{\operatorname{det} T}\left(T\left(\nabla\left(\nabla \cdot \cdot_{T} \boldsymbol{A}\right)\right)-\Delta_{T} \boldsymbol{A}\right)_{i} .
\end{aligned}
$$

Obviamente, na ausência de fontes externas, essas duas equações se transformam uma na outra quando trocamos $\boldsymbol{E}$ por $\boldsymbol{H}$ ou $\boldsymbol{B}$ por $\boldsymbol{D}$ e $\varepsilon_{\mathrm{r}}$ por $\mu_{\mathrm{r}}$.

Evidencia-se que todas essas equações são equações diferenciais parciais de segunda ordem a coeficientes constantes que não contêm termos de ordem inferior, nem segundas derivadas mistas entre a coordenada temporal $t$ e as coordenadas espaciais: poderíamos chamá-las de versões anisotrópicas da equação de onda usual. Para um melhor entendimento, vamos investigar o caso especial quando $\varepsilon_{\mathrm{r}}$ e $\mu_{\mathrm{r}}$ comutam, e portanto podemos escolher coordenadas cartesianas $x_{1}, x_{2}, x_{3}$ nas quais ambos são diagonais, conforme indicado na equação (3.6). Abreviando as derivadas parciais $c^{-1} \partial / \partial t$ e $\partial / \partial x_{i}$ por $\partial_{0}$ e $\partial_{i}(i=1,2,3)$, calculamos

$$
\left[\varepsilon_{\mathrm{r}} \boldsymbol{\nabla}\left(\boldsymbol{\nabla} \cdot\left(\varepsilon_{\mathrm{r}} \boldsymbol{E}\right)\right)\right]_{i}=\varepsilon_{i} \varepsilon_{j} \partial_{i} \partial_{j} E_{j},
$$

ou seja,

$$
\begin{aligned}
& {\left[\varepsilon_{\mathrm{r}} \boldsymbol{\nabla}\left(\boldsymbol{\nabla} \cdot\left(\varepsilon_{\mathrm{r}} \boldsymbol{E}\right)\right)\right]_{1}=\varepsilon_{1}^{2} \partial_{1}^{2} E_{1}+\varepsilon_{1} \varepsilon_{2} \partial_{1} \partial_{2} E_{2}+\varepsilon_{1} \varepsilon_{3} \partial_{1} \partial_{3} E_{3}} \\
& {\left[\varepsilon_{\mathrm{r}} \boldsymbol{\nabla}\left(\boldsymbol{\nabla} \cdot\left(\varepsilon_{\mathrm{r}} \boldsymbol{E}\right)\right)\right]_{2}=\varepsilon_{1} \varepsilon_{2} \partial_{1} \partial_{2} E_{1}+\varepsilon_{2}^{2} \partial_{2}^{2} E_{2}+\varepsilon_{2} \varepsilon_{3} \partial_{2} \partial_{3} E_{3}} \\
& {\left[\varepsilon_{\mathrm{r}} \boldsymbol{\nabla}\left(\boldsymbol{\nabla} \cdot\left(\varepsilon_{\mathrm{r}} \boldsymbol{E}\right)\right)\right]_{3}=\varepsilon_{1} \varepsilon_{3} \partial_{1} \partial_{3} E_{1}+\varepsilon_{2} \varepsilon_{3} \partial_{2} \partial_{3} E_{2}+\varepsilon_{3}^{2} \partial_{3}^{2} E_{3}}
\end{aligned}
$$


e

$$
\left[\boldsymbol{\nabla} \times\left(\mu_{\mathrm{r}}^{-1} \boldsymbol{\nabla} \times \boldsymbol{E}\right)\right]_{i}=\epsilon_{i j k} \mu_{k}^{-1} \partial_{j}(\boldsymbol{\nabla} \times \boldsymbol{E})_{k}=\epsilon_{i j k} \mu_{k}^{-1} \epsilon_{k l m} \partial_{j} \partial_{l} E_{m}
$$

ou seja,

$$
\begin{aligned}
& {\left[\boldsymbol{\nabla} \times\left(\mu_{\mathrm{r}}^{-1} \boldsymbol{\nabla} \times \boldsymbol{E}\right)\right]_{1}=\mu_{3}^{-1} \partial_{2} \partial_{1} E_{2}+\mu_{2}^{-1} \partial_{3} \partial_{1} E_{3}-\mu_{3}^{-1} \partial_{2}^{2} E_{1}-\mu_{2}^{-1} \partial_{3}^{2} E_{1},} \\
& {\left[\boldsymbol{\nabla} \times\left(\mu_{\mathrm{r}}^{-1} \boldsymbol{\nabla} \times \boldsymbol{E}\right)\right]_{2}=\mu_{1}^{-1} \partial_{3} \partial_{2} E_{3}+\mu_{3}^{-1} \partial_{1} \partial_{2} E_{1}-\mu_{1}^{-1} \partial_{3}^{2} E_{2}-\mu_{3}^{-1} \partial_{1}^{2} E_{2},} \\
& {\left[\boldsymbol{\nabla} \times\left(\mu_{\mathrm{r}}^{-1} \boldsymbol{\nabla} \times \boldsymbol{E}\right)\right]_{3}=\mu_{2}^{-1} \partial_{1} \partial_{3} E_{1}+\mu_{1}^{-1} \partial_{2} \partial_{3} E_{2}-\mu_{2}^{-1} \partial_{1}^{2} E_{3}-\mu_{1}^{-1} \partial_{2}^{2} E_{3},}
\end{aligned}
$$

e portanto o operador diferencial matricial

$$
D_{E}=\left(\begin{array}{ccc}
\left(D_{E}\right)_{11} & \left(D_{E}\right)_{12} & \left(D_{E}\right)_{13} \\
\left(D_{E}\right)_{21} & \left(D_{E}\right)_{22} & \left(D_{E}\right)_{23} \\
\left(D_{E}\right)_{31} & \left(D_{E}\right)_{32} & \left(D_{E}\right)_{33}
\end{array}\right)
$$

agindo sobre o campo $\boldsymbol{E}$ no lado esquerdo da equação (3.20) é explicitamente dado por

$$
\begin{aligned}
& \left(D_{E}\right)_{11}=\varepsilon_{1} \partial_{0}^{2}-\lambda \varepsilon_{1}^{2} \partial_{1}^{2}-\mu_{3}^{-1} \partial_{2}^{2}-\mu_{2}^{-1} \partial_{3}^{2}, \\
& \left(D_{E}\right)_{22}=\varepsilon_{2} \partial_{0}^{2}-\lambda \varepsilon_{2}^{2} \partial_{2}^{2}-\mu_{3}^{-1} \partial_{1}^{2}-\mu_{1}^{-1} \partial_{3}^{2}, \\
& \left(D_{E}\right)_{33}=\varepsilon_{3} \partial_{0}^{2}-\lambda \varepsilon_{3}^{2} \partial_{3}^{2}-\mu_{2}^{-1} \partial_{1}^{2}-\mu_{1}^{-1} \partial_{2}^{2},
\end{aligned}
$$

e

$$
\begin{aligned}
& \left(D_{E}\right)_{12}=\left(D_{E}\right)_{21}=\left(\mu_{3}^{-1}-\lambda \varepsilon_{1} \varepsilon_{2}\right) \partial_{1} \partial_{2}, \\
& \left(D_{E}\right)_{13}=\left(D_{E}\right)_{31}=\left(\mu_{2}^{-1}-\lambda \varepsilon_{1} \varepsilon_{3}\right) \partial_{1} \partial_{3}, \\
& \left(D_{E}\right)_{23}=\left(D_{E}\right)_{32}=\left(\mu_{1}^{-1}-\lambda \varepsilon_{2} \varepsilon_{3}\right) \partial_{2} \partial_{3} .
\end{aligned}
$$

De modo completamente análogo, o operador diferencial matricial

$$
D_{H}=\left(\begin{array}{ccc}
\left(D_{H}\right)_{11} & \left(D_{H}\right)_{12} & \left(D_{H}\right)_{13} \\
\left(D_{H}\right)_{21} & \left(D_{H}\right)_{22} & \left(D_{H}\right)_{23} \\
\left(D_{H}\right)_{31} & \left(D_{H}\right)_{32} & \left(D_{H}\right)_{33}
\end{array}\right)
$$

agindo sobre o campo $\boldsymbol{H}$ no lado esquerdo da equação (3.23) é explicitamente dado por

$$
\begin{aligned}
& \left(D_{H}\right)_{11}=\mu_{1} \partial_{0}^{2}-\lambda \mu_{1}^{2} \partial_{1}^{2}-\varepsilon_{3}^{-1} \partial_{2}^{2}-\varepsilon_{2}^{-1} \partial_{3}^{2}, \\
& \left(D_{H}\right)_{22}=\mu_{2} \partial_{0}^{2}-\lambda \mu_{2}^{2} \partial_{2}^{2}-\varepsilon_{3}^{-1} \partial_{1}^{2}-\varepsilon_{1}^{-1} \partial_{3}^{2}, \\
& \left(D_{H}\right)_{33}=\mu_{3} \partial_{0}^{2}-\lambda \mu_{3}^{2} \partial_{3}^{2}-\varepsilon_{2}^{-1} \partial_{1}^{2}-\varepsilon_{1}^{-1} \partial_{2}^{2},
\end{aligned}
$$


$\mathrm{e}$

$$
\begin{aligned}
& \left(D_{H}\right)_{12}=\left(D_{H}\right)_{21}=\left(\varepsilon_{3}^{-1}-\lambda \mu_{1} \mu_{2}\right) \partial_{1} \partial_{2}, \\
& \left(D_{H}\right)_{13}=\left(D_{H}\right)_{31}=\left(\varepsilon_{2}^{-1}-\lambda \mu_{1} \mu_{3}\right) \partial_{1} \partial_{3}, \\
& \left(D_{H}\right)_{23}=\left(D_{H}\right)_{32}=\left(\varepsilon_{1}^{-1}-\lambda \mu_{2} \mu_{3}\right) \partial_{2} \partial_{3} .
\end{aligned}
$$

É interessante notar que estas matrizes são simétricas, e que no limite do eletromagnetismo no vácuo, onde $\varepsilon_{\mathrm{r}}=1$ e $\mu_{\mathrm{r}}=1$, e com a escolha $\lambda=1$, $D_{E}$ e $D_{H}$ se reduzem ao produto $\square \otimes 1$, onde $\square$ é o operador de ondas comum e 1 é a matriz identidade $3 \times 3$.

\subsection{Equações de onda para os potenciais}

Devido ao fato de que as equações de Maxwell homogêneas permanecem inalteradas quando passamos do eletromagnetismo no vácuo para o eletromagnetismo em meios, continuamos podendo descrever a teoria inteiramente em termos de potenciais, ou mais precisamente, do potencial escalar $\phi$ e do potencial vetorial $\boldsymbol{A}$. Primeiro, sabemos do cálculo vetorial que a terceira equação de Maxwell (3.15-c) (ausência de cargas magnéticas) implica que $\boldsymbol{B}$ é o rotacional de algum campo vetorial $\boldsymbol{A}$, chamado de potencial vetorial:

$$
\boldsymbol{B}=\boldsymbol{\nabla} \times \boldsymbol{A} .
$$

Segundo, inserindo esta relação na segunda equação de Maxwell (3.15-b) (lei de Faraday), podemos reescrever esta na forma

$$
\boldsymbol{\nabla} \times\left(\boldsymbol{E}+\varkappa \frac{\partial \boldsymbol{A}}{\partial t}\right)=0
$$

e sabemos do cálculo vetorial que esta equação implica que a combinação $\boldsymbol{E}+\varkappa \partial \boldsymbol{A} / \partial t$ é (a menos de um sinal que é mera convenção) o gradiente de algum campo escalar $\phi$, chamado de potencial escalar:

$$
\boldsymbol{E}+\varkappa \frac{\partial \boldsymbol{A}}{\partial t}=-\nabla \phi,
$$

de modo que podemos escrever $\boldsymbol{E}$ em termos dos potenciais como

$$
\boldsymbol{E}=-\boldsymbol{\nabla} \phi-\varkappa \frac{\partial \boldsymbol{A}}{\partial t} .
$$

Agora, inserimos estas relações nas equações de Maxwell não homogêneas, usando ainda as relações constitutivas (3.1), (3.2). Substituindo a equação 
(3.37), em conjunto com a relação constitutiva $\boldsymbol{D}=\varepsilon \boldsymbol{E}=\varepsilon_{0} \varepsilon_{\mathrm{r}} \boldsymbol{E}$, na primeira equação de Maxwell (3.15-a) (lei de Gauss), obtemos

$$
-\varkappa \frac{\partial}{\partial t}\left(\boldsymbol{\nabla} \cdot\left(\varepsilon_{\mathrm{r}} \boldsymbol{A}\right)\right)-\boldsymbol{\nabla} \cdot\left(\varepsilon_{\mathrm{r}} \boldsymbol{\nabla} \phi\right)=\frac{\rho_{\mathrm{ext}}}{\epsilon_{0}},
$$

enquanto que substituindo as duas equações (3.36) e (3.37), em conjunto com a relação constitutiva $\boldsymbol{B}=\mu \boldsymbol{H}=\mu_{0} \mu_{\mathrm{r}} \boldsymbol{H}$ e ainda a equação (3.10), na quarta equação de Maxwell (3.15-d) (lei de Ampère), obtemos

$$
\boldsymbol{\nabla} \times\left(\mu_{\mathrm{r}}^{-1} \boldsymbol{\nabla} \times \boldsymbol{A}\right)=\varkappa \mu_{0} \boldsymbol{j}_{\mathrm{ext}}-\frac{1}{\varkappa c^{2}} \varepsilon_{\mathrm{r}} \boldsymbol{\nabla} \frac{\partial \phi}{\partial t}-\frac{1}{c^{2}} \varepsilon_{\mathrm{r}} \frac{\partial^{2} \boldsymbol{A}}{\partial t^{2}} .
$$

Essas equações podem ser simplificadas se usarmos a liberdade que ainda existe na escolha de $\boldsymbol{A}$ e $\phi$ para assujeitar estes potenciais a uma condição de calibre, sendo que a mais adequada no presente caso é a seguinte condição de calibre de Lorenz modificada, ${ }^{2}$

$$
\frac{1}{\varkappa c^{2}} \frac{\partial \phi}{\partial t}+\nabla \cdot{ }_{\varepsilon} \boldsymbol{A}=0 .
$$

Com isso, a equação (3.38) assume a forma

$$
\frac{1}{c^{2}} \frac{\partial^{2} \phi}{\partial t^{2}}-\Delta_{\varepsilon} \phi=\frac{\rho_{\mathrm{ext}}}{\varepsilon_{0}}
$$

que é claramente uma equação de onda para o potencial escalar $\phi$, enquanto que a equação (3.39) assume a forma

$$
\frac{1}{c^{2}} \frac{\partial^{2} \boldsymbol{A}}{\partial t^{2}}-\nabla\left(\boldsymbol{\nabla} \cdot \varepsilon_{\varepsilon} \boldsymbol{A}\right)+\varepsilon_{\mathrm{r}}^{-1} \nabla \times\left(\mu_{\mathrm{r}}^{-1} \nabla \times \boldsymbol{A}\right)=\varkappa \mu_{0} \varepsilon_{\mathrm{r}}^{-1} \boldsymbol{j}_{\mathrm{ext}} .
$$

Verifica-se então que o operador diferencial matricial $D_{A}$ agindo sobre o campo $\boldsymbol{A}$ no lado esquerdo da equação (3.42) é exatamente o mesma que o operador diferencial matricial $D_{E}$ agindo sobre o campo $\boldsymbol{E}$ no lado esquerdo da equação (3.20) na seção anterior, com a escolha específica $\lambda=1$, e portanto é explicitamente dado por

$$
D_{A}=\left(\begin{array}{lll}
\left(D_{A}\right)_{11} & \left(D_{A}\right)_{12} & \left(D_{A}\right)_{13} \\
\left(D_{A}\right)_{21} & \left(D_{A}\right)_{22} & \left(D_{A}\right)_{23} \\
\left(D_{A}\right)_{31} & \left(D_{A}\right)_{32} & \left(D_{A}\right)_{33}
\end{array}\right)
$$

\footnotetext{
${ }^{2} \mathrm{~A}$ inclusão do tensor $\varepsilon_{\mathrm{r}}$ nesta equação em nada altera o argumento usado para provar que esta é de fato uma legítima condição de calibre, no sentido de que, dado potenciais $\boldsymbol{A}^{\prime}$ e $\phi^{\prime}$ quaisquer satisfazendo apenas as equações (3.36) e (3.37), é sempre possível encontrar uma transformação de calibre $\boldsymbol{A}^{\prime} \longrightarrow \boldsymbol{A}=\boldsymbol{A}^{\prime}-\boldsymbol{\nabla} \chi, \phi^{\prime} \longrightarrow \phi=\phi^{\prime}+\varkappa \partial \chi / \partial t$ tal que os novos potenciais $\boldsymbol{A}$ e $\phi$ satisfaçam, além das mesmas equações (3.36) e (3.37), também a condição de calibre (3.40).
} 
onde

$$
\begin{aligned}
& \left(D_{A}\right)_{11}=\varepsilon_{1} \partial_{0}^{2}-\varepsilon_{1}^{2} \partial_{1}^{2}-\mu_{3}^{-1} \partial_{2}^{2}-\mu_{2}^{-1} \partial_{3}^{2}, \\
& \left(D_{A}\right)_{22}=\varepsilon_{2} \partial_{0}^{2}-\varepsilon_{2}^{2} \partial_{2}^{2}-\mu_{3}^{-1} \partial_{1}^{2}-\mu_{1}^{-1} \partial_{3}^{2}, \\
& \left(D_{A}\right)_{33}=\varepsilon_{3} \partial_{0}^{2}-\varepsilon_{3}^{2} \partial_{3}^{2}-\mu_{2}^{-1} \partial_{1}^{2}-\mu_{1}^{-1} \partial_{2}^{2},
\end{aligned}
$$

e

$$
\begin{aligned}
& \left(D_{A}\right)_{12}=\left(D_{A}\right)_{21}=\left(\mu_{3}^{-1}-\varepsilon_{1} \varepsilon_{2}\right) \partial_{1} \partial_{2}, \\
& \left(D_{A}\right)_{13}=\left(D_{A}\right)_{31}=\left(\mu_{2}^{-1}-\varepsilon_{1} \varepsilon_{3}\right) \partial_{1} \partial_{3}, \\
& \left(D_{A}\right)_{23}=\left(D_{A}\right)_{32}=\left(\mu_{1}^{-1}-\varepsilon_{2} \varepsilon_{3}\right) \partial_{2} \partial_{3} .
\end{aligned}
$$

Notamos que o operador $D_{A}$ é o mesmo que o operador $D_{E}$ da seção anterior, com a escolha específica $\lambda=1$, e no limite do eletromagnetismo no vácuo, onde $\varepsilon_{\mathrm{r}}=1$ e $\mu_{\mathrm{r}}=1$, se reduz ao produto $\square \otimes 1$, onde $\square$ é o operador de ondas comum e 1 é a matriz identidade $3 \times 3$.

\subsection{Propagação de ondas}

Nosso próximo objetivo é analisar a propagação de ondas eletromagnéticas planas em meios que possam exibir anisotropia, descrita por um tensor dielétrico $\varepsilon$ e/ou um tensor de permeabilidade magnética $\mu$, como antes. Para tanto, eliminamos os termos de fonte contendo $\rho_{\text {ext }}$ e $\boldsymbol{j}_{\text {ext }}$ e supomos que os campos $\boldsymbol{E}, \boldsymbol{D}, \boldsymbol{H}, \boldsymbol{B}$ tenham a forma

$$
\begin{aligned}
& \boldsymbol{E}(t, \boldsymbol{x})=\boldsymbol{E}_{0} \exp (-i(\omega t-\boldsymbol{k} \cdot \boldsymbol{x})), \\
& \boldsymbol{D}(t, \boldsymbol{x})=\boldsymbol{D}_{0} \exp (-i(\omega t-\boldsymbol{k} \cdot \boldsymbol{x})), \\
& \boldsymbol{H}(t, \boldsymbol{x})=\boldsymbol{H}_{0} \exp (-i(\omega t-\boldsymbol{k} \cdot \boldsymbol{x})), \\
& \boldsymbol{B}(t, \boldsymbol{x})=\boldsymbol{B}_{0} \exp (-i(\omega t-\boldsymbol{k} \cdot \boldsymbol{x})),
\end{aligned}
$$

onde $\omega$ é a frequência angular e $\boldsymbol{k}=\left(k_{1}, k_{2}, k_{3}\right)$ o vetor de onda, com módulo $|\boldsymbol{k}|$ igual ao inverso do comprimento de onda $\lambda$. Aqui, subentende-se, como é de costume, que os vetores coeficiente $\boldsymbol{E}_{0}, \boldsymbol{D}_{0}, \boldsymbol{H}_{0}, \boldsymbol{B}_{0}$ podem ser complexos e que no final deve ser tomado apenas a parte real do lado direito nestas equações: porém, essa operação final é suprimida da notação. ${ }^{3}$

\footnotetext{
${ }^{3}$ Quando o vetor coeficiente for real, essa prescrição implica que o sinal de $\omega$ não importa, pois cos é uma função par, e portanto suporemos sempre que $\omega$ é positiva, excluindo ainda o caso $\omega=0$ que corresponde a um campo estático e não a qualquer tipo de onda.
} 
Inserindo as equações (3.46)-(3.49) nas equações de Maxwell (3.15-a)(3.15-d) (sem fontes), obtemos

$$
\begin{gathered}
\boldsymbol{k} \cdot \boldsymbol{D}_{0}=0, \\
\boldsymbol{k} \times \boldsymbol{E}_{0}=\varkappa \omega \boldsymbol{B}_{0}, \\
\boldsymbol{k} \cdot \boldsymbol{B}_{0}=0, \\
\boldsymbol{k} \times \boldsymbol{H}_{0}=-\varkappa \omega \boldsymbol{D}_{0},
\end{gathered}
$$

Note que (supondo $\omega \neq 0$ ) a equação $(3.50-\mathrm{a})$ é consequência imediata da equação (3.50-d) e a equação (3.50-c) é consequência imediata da equação (3.50-b): os campos $\boldsymbol{D}$ e $\boldsymbol{B}$ oscilam no plano ortogonal à direção de propagação dada por $\boldsymbol{k}$. Por outro lado, isso não é geralmente verdade para os campos $\boldsymbol{E}$ e $\boldsymbol{H}$, mas usando as relações constitutivas (3.1), (3.2) em conjunto com a hipótese de que as matrizes $\varepsilon_{\mathrm{r}}$ e $\mu_{\mathrm{r}}$ são simétricas, podemos concluir que o campo $\boldsymbol{E}$ oscila no plano ortogonal ao vetor $\varepsilon_{\mathrm{r}} \boldsymbol{k}$ e o campo $\boldsymbol{H}$ oscila no plano ortogonal ao vetor $\mu_{\mathrm{r}} \boldsymbol{k}$. É neste sentido mais geral que continua valendo a afirmação de que ondas eletromagnéticas são transversais. Além disso, segue ainda que os campos $\boldsymbol{E}$ e $\boldsymbol{B}$, assim como os campos $\boldsymbol{D}$ e $\boldsymbol{H}$, são sempre ortogonais.

Também podemos inserir as mesmas equações (3.46) e (3.48) nas equações de onda (3.20) e (3.23) (sem fontes) para obter

$$
\frac{\omega^{2}}{c^{2}} \varepsilon_{\mathrm{r}} \boldsymbol{E}_{0}-\lambda\left(\varepsilon_{\mathrm{r}} \boldsymbol{k} \cdot \boldsymbol{E}_{0}\right) \varepsilon_{\mathrm{r}} \boldsymbol{k}+\boldsymbol{k} \times\left(\mu_{\mathrm{r}}^{-1} \boldsymbol{k} \times \boldsymbol{E}_{0}\right)=0,
$$

$\mathrm{e}$

$$
\frac{\omega^{2}}{c^{2}} \mu_{\mathrm{r}} \boldsymbol{H}_{0}-\lambda\left(\mu_{\mathrm{r}} \boldsymbol{k} \cdot \boldsymbol{H}_{0}\right) \mu_{\mathrm{r}} \boldsymbol{k}+\boldsymbol{k} \times\left(\varepsilon_{\mathrm{r}}^{-1} \boldsymbol{k} \times \boldsymbol{H}_{0}\right)=0,
$$

respectivamente. Isso corresponde à exigência de que $\boldsymbol{E}_{0}$ e $\boldsymbol{H}_{0}$ sejam soluções das equações lineares

$$
\sigma_{D_{E}} \boldsymbol{E}_{0}=0
$$

$\mathrm{e}$

$$
\sigma_{D_{H}} \boldsymbol{H}_{0}=0
$$

onde $\sigma_{D_{E}}$ e $\sigma_{D_{H}}$ são os símbolos principais dos operadores $D_{E}$ e $D_{H}$, respectivamente, i.e.,

$$
\sigma_{D_{E}}=\left(\begin{array}{ccc}
\left(\sigma_{E}\right)_{11} & \left(\sigma_{E}\right)_{12} & \left(\sigma_{E}\right)_{13} \\
\left(\sigma_{E}\right)_{21} & \left(\sigma_{E}\right)_{22} & \left(\sigma_{E}\right)_{23} \\
\left(\sigma_{E}\right)_{31} & \left(\sigma_{E}\right)_{32} & \left(\sigma_{E}\right)_{33}
\end{array}\right)
$$


com

$$
\begin{aligned}
& \left(\sigma_{E}\right)_{11}(\omega, \boldsymbol{k}, \lambda)=\varepsilon_{1} \omega^{2} / c^{2}-\lambda \varepsilon_{1}^{2} k_{1}^{2}-\mu_{3}^{-1} k_{2}^{2}-\mu_{2}^{-1} k_{3}^{2}, \\
& \left(\sigma_{E}\right)_{22}(\omega, \boldsymbol{k}, \lambda)=\varepsilon_{2} \omega^{2} / c^{2}-\lambda \varepsilon_{2}^{2} k_{2}^{2}-\mu_{3}^{-1} k_{1}^{2}-\mu_{1}^{-1} k_{3}^{2}, \\
& \left(\sigma_{E}\right)_{33}(\omega, \boldsymbol{k}, \lambda)=\varepsilon_{3} \omega^{2} / c^{2}-\lambda \varepsilon_{3}^{2} k_{3}^{2}-\mu_{2}^{-1} k_{1}^{2}-\mu_{1}^{-1} k_{2}^{2},
\end{aligned}
$$

$\mathrm{e}$

$$
\begin{aligned}
& \left(\sigma_{E}\right)_{12}(\omega, \boldsymbol{k}, \lambda)=\left(\sigma_{E}\right)_{21}(\omega, \boldsymbol{k}, \lambda)=\left(\mu_{3}^{-1}-\lambda \varepsilon_{1} \varepsilon_{2}\right) k_{1} k_{2}, \\
& \left(\sigma_{E}\right)_{13}(\omega, \boldsymbol{k}, \lambda)=\left(\sigma_{E}\right)_{31}(\omega, \boldsymbol{k}, \lambda)=\left(\mu_{2}^{-1}-\lambda \varepsilon_{1} \varepsilon_{3}\right) k_{1} k_{3}, \\
& \left(\sigma_{E}\right)_{23}(\omega, \boldsymbol{k}, \lambda)=\left(\sigma_{E}\right)_{32}(\omega, \boldsymbol{k}, \lambda)=\left(\mu_{1}^{-1}-\lambda \varepsilon_{2} \varepsilon_{3}\right) k_{2} k_{3},
\end{aligned}
$$

e analogamente

$$
\sigma_{D_{H}}=\left(\begin{array}{ccc}
\left(\sigma_{H}\right)_{11} & \left(\sigma_{H}\right)_{12} & \left(\sigma_{H}\right)_{13} \\
\left(\sigma_{H}\right)_{21} & \left(\sigma_{H}\right)_{22} & \left(\sigma_{H}\right)_{23} \\
\left(\sigma_{H}\right)_{31} & \left(\sigma_{H}\right)_{32} & \left(\sigma_{H}\right)_{33}
\end{array}\right)
$$

com

$$
\begin{aligned}
& \left(\sigma_{H}\right)_{11}(\omega, \boldsymbol{k}, \lambda)=\mu_{1} \omega^{2} / c^{2}-\lambda \mu_{1}^{2} k_{1}^{2}-\varepsilon_{3}^{-1} k_{2}^{2}-\varepsilon_{2}^{-1} k_{3}^{2}, \\
& \left(\sigma_{H}\right)_{22}(\omega, \boldsymbol{k}, \lambda)=\mu_{2} \omega^{2} / c^{2}-\lambda \mu_{2}^{2} k_{2}^{2}-\varepsilon_{3}^{-1} k_{1}^{2}-\varepsilon_{1}^{-1} k_{3}^{2}, \\
& \left(\sigma_{H}\right)_{33}(\omega, \boldsymbol{k}, \lambda)=\mu_{3} \omega^{2} / c^{2}-\lambda \mu_{3}^{2} k_{3}^{2}-\varepsilon_{2}^{-1} k_{1}^{2}-\varepsilon_{1}^{-1} k_{2}^{2},
\end{aligned}
$$

$\mathrm{e}$

$$
\begin{aligned}
& \left(\sigma_{H}\right)_{12}(\omega, \boldsymbol{k}, \lambda)=\left(\sigma_{H}\right)_{21}(\omega, \boldsymbol{k}, \lambda)=\left(\varepsilon_{3}^{-1}-\lambda \mu_{1} \mu_{2}\right) k_{1} k_{2}, \\
& \left(\sigma_{H}\right)_{13}(\omega, \boldsymbol{k}, \lambda)=\left(\sigma_{H}\right)_{31}(\omega, \boldsymbol{k}, \lambda)=\left(\varepsilon_{2}^{-1}-\lambda \mu_{1} \mu_{3}\right) k_{1} k_{3}, \\
& \left(\sigma_{H}\right)_{23}(\omega, \boldsymbol{k}, \lambda)=\left(\sigma_{H}\right)_{32}(\omega, \boldsymbol{k}, \lambda)=\left(\varepsilon_{1}^{-1}-\lambda \mu_{2} \mu_{3}\right) k_{2} k_{3} .
\end{aligned}
$$

Observamos, novamente, que as equações e relações para $\boldsymbol{E}$ e para $\boldsymbol{H}$ se transformam umas nas outras quando trocarmos $\boldsymbol{E}$ por $\boldsymbol{H}$ e $\varepsilon$ por $\mu$; portanto, basta no que segue analisar a primeira delas.

Conforme um teorema padrão de álgebra linear, o sistema linear (3.53) homogêneo tem uma solução $\boldsymbol{E}_{0}$ não-trivial se e somente se o determinante da matriz $\sigma_{D_{E}}$ for nulo,

$$
\operatorname{det} \sigma_{D_{E}}(\omega, \boldsymbol{k}, \lambda)=0,
$$

i.e., se e somente se o vetor $(\omega / c, \boldsymbol{k})$ no espaço $\mathbb{R}^{4}$ de Minkowski for um vetor característico para o operador $D_{E}$. Para simplificar os cálculos, vamos analisar primeiro o caso $\lambda=0$ e em seguida mostrar que se um vetor $\boldsymbol{E}_{0}$ 
pertencer ao núcleo da matriz $\sigma_{D_{E}}(\omega, \boldsymbol{k}, 0)$, o que obviamente só é possível se valer

$$
\operatorname{det} \sigma_{D_{E}}(\omega, \boldsymbol{k}, 0)=0
$$

então $\boldsymbol{E}_{0}$ é automaticamente ortogonal ao vetor $\varepsilon_{\mathrm{r}} \boldsymbol{k}$ e portanto também pertence ao núcleo da matriz $\sigma_{D_{E}}(\omega, \boldsymbol{k}, \lambda)$, para qualquer valor de $\lambda$. Para executar este cálculo, notamos que

$$
\sigma_{D_{E}}(\omega, \boldsymbol{k}, 0)=\frac{\omega^{2}}{c^{2}} \varepsilon_{\mathrm{r}}+\sigma_{D_{E}}^{\prime}(\boldsymbol{k})
$$

onde $\sigma_{D_{E}}^{\prime}(\boldsymbol{k})$ é a matriz

$$
\sigma_{D_{E}}^{\prime}(\boldsymbol{k})=\left(\begin{array}{ccc}
-\mu_{3}^{-1} k_{2}^{2}-\mu_{2}^{-1} k_{3}^{2} & \mu_{3}^{-1} k_{1} k_{2} & \mu_{2}^{-1} k_{1} k_{3} \\
\mu_{3}^{-1} k_{1} k_{2} & -\mu_{3}^{-1} k_{1}^{2}-\mu_{1}^{-1} k_{3}^{2} & \mu_{1}^{-1} k_{2} k_{3} \\
\mu_{2}^{-1} k_{1} k_{3} & \mu_{1}^{-1} k_{2} k_{3} & -\mu_{2}^{-1} k_{1}^{2}-\mu_{1}^{-1} k_{2}^{2}
\end{array}\right)
$$

que é degenerada porque, obviamente, satisfaz a seguinte relação:

$$
\sigma_{D_{E}}^{\prime}(\boldsymbol{k}) \boldsymbol{k}=0
$$


Segue que

$$
\begin{aligned}
& \operatorname{det} \sigma_{D_{E}}(\omega, \boldsymbol{k}, 0)=\left(\varepsilon_{1} \frac{\omega^{2}}{c^{2}}-\mu_{3}^{-1} k_{2}^{2}-\mu_{2}^{-1} k_{3}^{2}\right) \\
& \times\left(\varepsilon_{2} \frac{\omega^{2}}{c^{2}}-\mu_{3}^{-1} k_{1}^{2}-\mu_{1}^{-1} k_{3}^{2}\right) \\
& \times\left(\varepsilon_{3} \frac{\omega^{2}}{c^{2}}-\mu_{2}^{-1} k_{1}^{2}-\mu_{1}^{-1} k_{2}^{2}\right) \\
& +2 \mu_{1}^{-1} \mu_{2}^{-1} \mu_{3}^{-1} k_{1}^{2} k_{2}^{2} k_{3}^{2} \\
& -\left(\varepsilon_{1} \frac{\omega^{2}}{c^{2}}-\mu_{3}^{-1} k_{2}^{2}-\mu_{2}^{-1} k_{3}^{2}\right) \mu_{1}^{-2} k_{2}^{2} k_{3}^{2} \\
& -\left(\varepsilon_{2} \frac{\omega^{2}}{c^{2}}-\mu_{3}^{-1} k_{1}^{2}-\mu_{1}^{-1} k_{3}^{2}\right) \mu_{2}^{-2} k_{1}^{2} k_{3}^{2} \\
& -\left(\varepsilon_{3} \frac{\omega^{2}}{c^{2}}-\mu_{2}^{-1} k_{1}^{2}-\mu_{1}^{-1} k_{2}^{2}\right) \mu_{3}^{-2} k_{1}^{2} k_{2}^{2} \\
& =\operatorname{det} \varepsilon_{\mathrm{r}} \frac{\omega^{6}}{c^{6}} \\
& -\operatorname{det} \varepsilon_{\mathrm{r}}\left(\varepsilon_{3}^{-1}\left(\mu_{2}^{-1} k_{1}^{2}+\mu_{1}^{-1} k_{2}^{2}\right)\right. \\
& +\varepsilon_{2}^{-1}\left(\mu_{3}^{-1} k_{1}^{2}+\mu_{1}^{-1} k_{3}^{2}\right) \\
& \left.+\varepsilon_{1}^{-1}\left(\mu_{3}^{-1} k_{2}^{2}+\mu_{2}^{-1} k_{3}^{2}\right)\right) \frac{\omega^{4}}{c^{4}} \\
& +\left(\varepsilon_{1}\left(\mu_{2}^{-1} k_{1}^{2}+\mu_{1}^{-1} k_{2}^{2}\right)\left(\mu_{3}^{-1} k_{1}^{2}+\mu_{1}^{-1} k_{3}^{2}\right)\right. \\
& +\varepsilon_{2}\left(\mu_{2}^{-1} k_{1}^{2}+\mu_{1}^{-1} k_{2}^{2}\right)\left(\mu_{3}^{-1} k_{2}^{2}+\mu_{2}^{-1} k_{3}^{2}\right) \\
& +\varepsilon_{3}\left(\mu_{3}^{-1} k_{1}^{2}+\mu_{1}^{-1} k_{3}^{2}\right)\left(\mu_{3}^{-1} k_{2}^{2}+\mu_{2}^{-1} k_{3}^{2}\right) \\
& \left.-\varepsilon_{3} \mu_{3}^{-2} k_{1}^{2} k_{2}^{2}-\varepsilon_{2} \mu_{2}^{-2} k_{1}^{2} k_{3}^{2}-\varepsilon_{1} \mu_{1}^{-2} k_{2}^{2} k_{3}^{2}\right) \frac{\omega^{2}}{c^{2}} \\
& -\left(\left(\mu_{3}^{-1} k_{2}^{2}+\mu_{2}^{-1} k_{3}^{2}\right)\left(\mu_{3}^{-1} k_{1}^{2}+\mu_{1}^{-1} k_{3}^{2}\right)\left(\mu_{2}^{-1} k_{1}^{2}+\mu_{1}^{-1} k_{2}^{2}\right)\right. \\
& -2 \mu_{1}^{-1} \mu_{2}^{-1} \mu_{3}^{-1} k_{1}^{2} k_{2}^{2} k_{3}^{2} \\
& -\mu_{2}^{-1} \mu_{3}^{-2} k_{1}^{4} k_{2}^{2}-\mu_{1}^{-1} \mu_{3}^{-2} k_{1}^{2} k_{2}^{4} \\
& -\mu_{3}^{-1} \mu_{2}^{-2} k_{1}^{4} k_{3}^{2}-\mu_{1}^{-1} \mu_{2}^{-2} k_{1}^{2} k_{3}^{4} \\
& \left.-\mu_{3}^{-1} \mu_{1}^{-2} k_{2}^{4} k_{3}^{2}-\mu_{2}^{-1} \mu_{1}^{-2} k_{2}^{2} k_{3}^{4}\right) \text {. }
\end{aligned}
$$

Claramente, neste determinante, escrito aqui como polinômio cúbico em 
$\omega^{2} / c^{2}$, o termo constante se anula, e obtemos

$$
\operatorname{det} \sigma_{D_{E}}(\omega, \boldsymbol{k}, 0)=\operatorname{det} \varepsilon_{\mathrm{r}} \frac{\omega^{2}}{c^{2}} \Delta(\omega, \boldsymbol{k})
$$

onde

$$
\Delta(\omega, \boldsymbol{k})=\frac{\omega^{4}}{c^{4}}-p(\boldsymbol{k}) \frac{\omega^{2}}{c^{2}}+q(\boldsymbol{k})
$$

com

$$
\begin{aligned}
p(\boldsymbol{k})= & \left(\varepsilon_{2}^{-1} \mu_{3}^{-1}+\varepsilon_{3}^{-1} \mu_{2}^{-1}\right) k_{1}^{2} \\
& +\left(\varepsilon_{3}^{-1} \mu_{1}^{-1}+\varepsilon_{1}^{-1} \mu_{3}^{-1}\right) k_{2}^{2} \\
& +\left(\varepsilon_{1}^{-1} \mu_{2}^{-1}+\varepsilon_{2}^{-1} \mu_{1}^{-1}\right) k_{3}^{2}
\end{aligned}
$$

e

$$
\begin{aligned}
q(\boldsymbol{k})= & \left(\varepsilon_{2}^{-1} \varepsilon_{3}^{-1} k_{1}^{2}+\varepsilon_{3}^{-1} \varepsilon_{1}^{-1} k_{2}^{2}+\varepsilon_{1}^{-1} \varepsilon_{2}^{-1} k_{3}^{2}\right) \\
& \times\left(\mu_{2}^{-1} \mu_{3}^{-1} k_{1}^{2}+\mu_{3}^{-1} \mu_{1}^{-1} k_{2}^{2}+\mu_{1}^{-1} \mu_{2}^{-1} k_{3}^{2}\right)
\end{aligned}
$$

pois

$$
\begin{aligned}
\varepsilon_{2}^{-1} \varepsilon_{3}^{-1}\left(\mu_{2}^{-1} k_{1}^{2}+\mu_{1}^{-1} k_{2}^{2}\right)\left(\mu_{3}^{-1} k_{1}^{2}+\mu_{1}^{-1} k_{3}^{2}\right) \\
+\varepsilon_{1}^{-1} \varepsilon_{3}^{-1}\left(\mu_{2}^{-1} k_{1}^{2}+\mu_{1}^{-1} k_{2}^{2}\right)\left(\mu_{3}^{-1} k_{2}^{2}+\mu_{2}^{-1} k_{3}^{2}\right) \\
+\varepsilon_{1}^{-1} \varepsilon_{2}^{-1}\left(\mu_{3}^{-1} k_{1}^{2}+\mu_{1}^{-1} k_{3}^{2}\right)\left(\mu_{3}^{-1} k_{2}^{2}+\mu_{2}^{-1} k_{3}^{2}\right) \\
-\varepsilon_{1}^{-1} \varepsilon_{2}^{-1} \mu_{3}^{-2} k_{1}^{2} k_{2}^{2}-\varepsilon_{1}^{-1} \varepsilon_{3}^{-1} \mu_{2}^{-2} k_{1}^{2} k_{3}^{2}-\varepsilon_{2}^{-1} \varepsilon_{3}^{-1} \mu_{1}^{-2} k_{2}^{2} k_{3}^{2} \\
=\varepsilon_{2}^{-1} \varepsilon_{3}^{-1} \mu_{2}^{-1} \mu_{3}^{-1} k_{1}^{4}+\varepsilon_{2}^{-1} \varepsilon_{3}^{-1} \mu_{1}^{-1} \mu_{3}^{-1} k_{1}^{2} k_{2}^{2}+\varepsilon_{2}^{-1} \varepsilon_{3}^{-1} \mu_{1}^{-1} \mu_{2}^{-1} k_{1}^{2} k_{3}^{2} \\
\quad+\varepsilon_{1}^{-1} \varepsilon_{3}^{-1} \mu_{2}^{-1} \mu_{3}^{-1} k_{1}^{2} k_{2}^{2}+\varepsilon_{1}^{-1} \varepsilon_{3}^{-1} \mu_{1}^{-1} \mu_{3}^{-1} k_{2}^{4}+\varepsilon_{1}^{-1} \varepsilon_{3}^{-1} \mu_{1}^{-1} \mu_{2}^{-1} k_{2}^{2} k_{3}^{2} \\
\quad+\varepsilon_{1}^{-1} \varepsilon_{2}^{-1} \mu_{2}^{-1} \mu_{3}^{-1} k_{1}^{2} k_{3}^{2}+\varepsilon_{1}^{-1} \varepsilon_{2}^{-1} \mu_{1}^{-1} \mu_{3}^{-1} k_{2}^{2} k_{3}^{2}+\varepsilon_{1}^{-1} \varepsilon_{2}^{-1} \mu_{1}^{-1} \mu_{2}^{-1} k_{3}^{4} \\
= \\
q(\boldsymbol{k}) .
\end{aligned}
$$

Nosso próximo objetivo é mostrar que, dado o vetor $\boldsymbol{k}$, a equação $\Delta(\omega, \boldsymbol{k})=0$, como equação quadrática em $\omega^{2} / c^{2}$, sempre tem duas soluções reais. Para tanto, calculamos o discriminante $p(\boldsymbol{k})^{2}-4 q(\boldsymbol{k})$, e mostraremos que é sempre $\geqslant 0$ : vale 


$$
\begin{aligned}
p(\boldsymbol{k})^{2} & -4 q(\boldsymbol{k}) \\
= & \left.\varepsilon_{2}^{-2} \mu_{3}^{-2}+2 \varepsilon_{2}^{-1} \varepsilon_{3}^{-1} \mu_{2}^{-1} \mu_{3}^{-1}+\varepsilon_{3}^{-2} \mu_{2}^{-2}\right) k_{1}^{4}-4 \varepsilon_{2}^{-1} \varepsilon_{3}^{-1} \mu_{2}^{-1} \mu_{3}^{-1} k_{1}^{4} \\
& +\left(\varepsilon_{3}^{-2} \mu_{1}^{-2}+2 \varepsilon_{3}^{-1} \varepsilon_{1}^{-1} \mu_{3}^{-1} \mu_{1}^{-1}+\varepsilon_{1}^{-2} \mu_{3}^{-2}\right) k_{2}^{4}-4 \varepsilon_{3}^{-1} \varepsilon_{1}^{-1} \mu_{3}^{-1} \mu_{1}^{-1} k_{2}^{4} \\
& +\left(\varepsilon_{1}^{-2} \mu_{2}^{-2}+2 \varepsilon_{1}^{-1} \varepsilon_{2}^{-1} \mu_{1}^{-1} \mu_{2}^{-1}+\varepsilon_{2}^{-2} \mu_{1}^{-2}\right) k_{3}^{4}-4 \varepsilon_{1}^{-1} \varepsilon_{2}^{-1} \mu_{1}^{-1} \mu_{2}^{-1} k_{3}^{4} \\
& +2\left(\varepsilon_{2}^{-1} \mu_{3}^{-1}+\varepsilon_{3}^{-1} \mu_{2}^{-1}\right)\left(\varepsilon_{3}^{-1} \mu_{1}^{-1}+\varepsilon_{1}^{-1} \mu_{3}^{-1}\right) k_{1}^{2} k_{2}^{2} \\
& -4\left(\varepsilon_{2}^{-1} \mu_{3}^{-1} \varepsilon_{3}^{-1} \mu_{1}^{-1}+\varepsilon_{3}^{-1} \mu_{2}^{-1} \varepsilon_{1}^{-1} \mu_{3}^{-1}\right) k_{1}^{2} k_{2}^{2} \\
& +2\left(\varepsilon_{3}^{-1} \mu_{1}^{-1}+\varepsilon_{1}^{-1} \mu_{3}^{-1}\right)\left(\varepsilon_{1}^{-1} \mu_{2}^{-1}+\varepsilon_{2}^{-1} \mu_{1}^{-1}\right) k_{2}^{2} k_{3}^{2} \\
& -4\left(\varepsilon_{3}^{-1} \mu_{1}^{-1} \varepsilon_{1}^{-1} \mu_{2}^{-1}+\varepsilon_{1}^{-1} \mu_{3}^{-1} \varepsilon_{2}^{-1} \mu_{1}^{-1}\right) k_{2}^{2} k_{3}^{2} \\
& +2\left(\varepsilon_{1}^{-1} \mu_{2}^{-1}+\varepsilon_{2}^{-1} \mu_{1}^{-1}\right)\left(\varepsilon_{2}^{-1} \mu_{3}^{-1}+\varepsilon_{3}^{-1} \mu_{2}^{-1}\right) k_{3}^{2} k_{1}^{2} \\
& -4\left(\varepsilon_{1}^{-1} \mu_{2}^{-1} \varepsilon_{2}^{-1} \mu_{3}^{-1}+\varepsilon_{2}^{-1} \mu_{1}^{-1} \varepsilon_{3}^{-1} \mu_{2}^{-1}\right) k_{3}^{2} k_{1}^{2} \\
= & \left(\varepsilon_{2}^{-2} \mu_{3}^{-2}-2 \varepsilon_{2}^{-1} \varepsilon_{3}^{-1} \mu_{2}^{-1} \mu_{3}^{-1}+\varepsilon_{3}^{-2} \mu_{2}^{-2}\right) k_{1}^{4} \\
& +\left(\varepsilon_{3}^{-2} \mu_{1}^{-2}-2 \varepsilon_{3}^{-1} \varepsilon_{1}^{-1} \mu_{3}^{-1} \mu_{1}^{-1}+\varepsilon_{1}^{-2} \mu_{3}^{-2}\right) k_{2}^{4} \\
& +\left(\varepsilon_{1}^{-2} \mu_{2}^{-2}-2 \varepsilon_{1}^{-1} \varepsilon_{2}^{-1} \mu_{1}^{-1} \mu_{2}^{-1}+\varepsilon_{2}^{-2} \mu_{1}^{-2}\right) k_{3}^{4} \\
& -2\left(\varepsilon_{2}^{-1} \mu_{3}^{-1}-\varepsilon_{3}^{-1} \mu_{2}^{-1}\right)\left(\varepsilon_{3}^{-1} \mu_{1}^{-1}-\varepsilon_{1}^{-1} \mu_{3}^{-1}\right) k_{1}^{2} k_{2}^{2} \\
& -2\left(\varepsilon_{3}^{-1} \mu_{1}^{-1}-\varepsilon_{1}^{-1} \mu_{3}^{-1}\right)\left(\varepsilon_{1}^{-1} \mu_{2}^{-1}-\varepsilon_{2}^{-1} \mu_{1}^{-1}\right) k_{2}^{2} k_{3}^{2} \\
& -2\left(\varepsilon_{1}^{-1} \mu_{2}^{-1}-\varepsilon_{2}^{-1} \mu_{1}^{-1}\right)\left(\varepsilon_{2}^{-1} \mu_{3}^{-1}-\varepsilon_{3}^{-1} \mu_{2}^{-1}\right) k_{3}^{2} k_{1}^{2}
\end{aligned}
$$

ou seja,

$$
\begin{aligned}
p(\boldsymbol{k})^{2}-4 q(\boldsymbol{k})= & \left(\left(\varepsilon_{2}^{-1} \mu_{3}^{-1}-\varepsilon_{3}^{-1} \mu_{2}^{-1}\right) k_{1}^{2}\right)^{2} \\
& +\left(\left(\varepsilon_{3}^{-1} \mu_{1}^{-1}-\varepsilon_{1}^{-1} \mu_{3}^{-1}\right) k_{2}^{2}\right)^{2} \\
& +\left(\left(\varepsilon_{1}^{-1} \mu_{2}^{-1}-\varepsilon_{2}^{-1} \mu_{1}^{-1}\right) k_{3}^{2}\right)^{2} \\
& -2\left(\varepsilon_{2}^{-1} \mu_{3}^{-1}-\varepsilon_{3}^{-1} \mu_{2}^{-1}\right)\left(\varepsilon_{3}^{-1} \mu_{1}^{-1}-\varepsilon_{1}^{-1} \mu_{3}^{-1}\right) k_{1}^{2} k_{2}^{2} \\
& -2\left(\varepsilon_{3}^{-1} \mu_{1}^{-1}-\varepsilon_{1}^{-1} \mu_{3}^{-1}\right)\left(\varepsilon_{1}^{-1} \mu_{2}^{-1}-\varepsilon_{2}^{-1} \mu_{1}^{-1}\right) k_{2}^{2} k_{3}^{2} \\
& -2\left(\varepsilon_{1}^{-1} \mu_{2}^{-1}-\varepsilon_{2}^{-1} \mu_{1}^{-1}\right)\left(\varepsilon_{2}^{-1} \mu_{3}^{-1}-\varepsilon_{3}^{-1} \mu_{2}^{-1}\right) k_{3}^{2} k_{1}^{2} .
\end{aligned}
$$

Essa é uma expressão do tipo $a^{2}+b^{2}+c^{2}-2 a b-2 a c-2 b c$, que a priori pode ser positiva ou negativa. Note, porém, que ela é sempre não-negativa quando os números $a, b$ e $c$ não têm todos o mesmo sinal, ou quando um deles é zero. De fato, se $c=0$, por exemplo, vale $a^{2}+b^{2}+c^{2}-2 a b-2 a c-2 b c=$ $a^{2}+b^{2}-2 a b=(a-b)^{2} \geqslant 0(=0$ apenas se $a=b)$, e se $a, b, c$ são todos $\neq 0$ e tais que $a$ e $b$ têm o mesmo sinal enquanto que $c$ tem o sinal oposto, 
por exemplo, vale $a c<0, b c<0$ e portanto $a^{2}+b^{2}+c^{2}-2 a b-2 a c-2 b c>$ $a^{2}+b^{2}+c^{2}-2 a b=(a-b)^{2}+c^{2}>0$. No presente caso, temos

$$
\left(\begin{array}{c}
a \\
b \\
c
\end{array}\right)=\left(\begin{array}{c}
\left(\varepsilon_{2}^{-1} \mu_{3}^{-1}-\varepsilon_{3}^{-1} \mu_{2}^{-1}\right) k_{1}^{2} \\
\left(\varepsilon_{3}^{-1} \mu_{1}^{-1}-\varepsilon_{1}^{-1} \mu_{3}^{-1}\right) k_{2}^{2} \\
\left(\varepsilon_{1}^{-1} \mu_{2}^{-1}-\varepsilon_{2}^{-1} \mu_{1}^{-1}\right) k_{3}^{2}
\end{array}\right)
$$

e se introduzirmos vetores $\varepsilon^{-1}$ e $\boldsymbol{\mu}^{-1}$ conforme

$$
\boldsymbol{\varepsilon}^{-1}=\left(\begin{array}{c}
\varepsilon_{1}^{-1} \\
\varepsilon_{2}^{-1} \\
\varepsilon_{3}^{-1}
\end{array}\right), \boldsymbol{\mu}^{-1}=\left(\begin{array}{c}
\mu_{1}^{-1} \\
\mu_{2}^{-1} \\
\mu_{3}^{-1}
\end{array}\right),
$$

obtemos

$$
\left(\begin{array}{l}
a \\
b \\
c
\end{array}\right)=\left(\begin{array}{c}
\left(\varepsilon^{-1} \times \mu^{-1}\right)_{1} k_{1}^{2} \\
\left(\varepsilon^{-1} \times \mu^{-1}\right)_{2} k_{2}^{2} \\
\left(\varepsilon^{-1} \times \mu^{-1}\right)_{3} k_{3}^{2}
\end{array}\right) .
$$

Vamos supor que as matrizes $\varepsilon$ e $\mu$ não são proporcionais, ou seja, os vetores $\varepsilon^{-1}$ e $\boldsymbol{\mu}^{-1}$ são linearmente independentes, i.e., $\boldsymbol{\varepsilon}^{-1} \times \boldsymbol{\mu}^{-1} \neq 0$, pois caso contrário, vale $p^{2}-4 q=0$, e obtemos duas raízes reais iguais para $\omega^{2} / c^{2}$. Agora, como as matrizes $\varepsilon$ e $\mu$ são positivas definidas, i.e., os seus autovalores $\varepsilon_{1}, \varepsilon_{2}, \varepsilon_{3}$ e $\mu_{1}, \mu_{2}, \mu_{3}$ são todos $>0$, e portanto o mesmo vale para os autovalores inversos $\varepsilon_{1}^{-1}, \varepsilon_{2}^{-1}, \varepsilon_{3}^{-1}$ e $\mu_{1}^{-1}, \mu_{2}^{-1}, \mu_{3}^{-1}$, as componentes do produto vetorial $\boldsymbol{\varepsilon}^{-1} \times \boldsymbol{\mu}^{-1}$ não podem ter todas o mesmo sinal. (De fato, se $\boldsymbol{u}$ e $\boldsymbol{v}$ são vetores linearmente independentes em $\mathbb{R}^{3}$ tais que as componentes $u_{1}, u_{2}, u_{3}$ de $\boldsymbol{u}$, ou as componentes $v_{1}, v_{2}, v_{3}$ de $\boldsymbol{v}$, têm todas o mesmo sinal, então as componentes do seu produto vetorial $\boldsymbol{u} \times \boldsymbol{v}$ não podem ter todas o mesmo sinal, pois se fossem todas $\geqslant 0$ ou todas $\leqslant 0$, com pelo menos uma $\neq 0$, o produto escalar de $\boldsymbol{u} \times \boldsymbol{v}$ com $\boldsymbol{u}$, ou com $\boldsymbol{v}$, teria que ser $>0$ ou $<0$, mas ele vale 0 .) Isso prova que a discriminante $p^{2}-4 q$ é sempre $\geqslant 0$.

Sendo assim, dado o vetor $\boldsymbol{k}$, a equação (3.62) tem duas soluções nãotriviais, $\omega_{+}$e $\omega_{-}$, dadas por

$$
\begin{aligned}
& \frac{\omega_{+}^{2}}{c^{2}}=\frac{1}{2}\left(p+\sqrt{p^{2}-4 q}\right), \\
& \frac{\omega_{-}^{2}}{c^{2}}=\frac{1}{2}\left(p-\sqrt{p^{2}-4 q}\right) .
\end{aligned}
$$

O fenômeno de termos duas frequências possíveis, $\omega_{+}$e $\omega_{-}$, para o mesmo vetor de onda $\boldsymbol{k}$, significa que também temos duas velocidades de propagação 
distintas, $v_{+}$e $v_{-}$, e dois índices de refração distintos, $n_{+}$e $n_{-}$, dados por

$$
\begin{array}{ll}
\frac{1}{n_{+}}=\frac{v_{+}}{c}=\frac{\omega_{+}}{c|\boldsymbol{k}|}=\frac{1}{2|\boldsymbol{k}|}\left(p+\sqrt{p^{2}-4 q}\right) & \text { (onda rápida) } \\
\frac{1}{n_{-}}=\frac{v_{-}}{c}=\frac{\omega_{-}}{c|\boldsymbol{k}|}=\frac{1}{2|\boldsymbol{k}|}\left(p-\sqrt{p^{2}-4 q}\right) \quad \text { (onda lenta). }
\end{array}
$$

Essas ondas são linearmente polarizadas em direções diferentes, pois os vetores $\boldsymbol{E}_{0}^{+}$e $\boldsymbol{E}_{0}^{-}$devem satisfazer as equações

$$
\begin{aligned}
& \sigma_{D_{E}}\left(\omega_{+}, \boldsymbol{k}, 0\right) \boldsymbol{E}_{0}^{+}=0, \\
& \sigma_{D_{E}}\left(\omega_{-}, \boldsymbol{k}, 0\right) \boldsymbol{E}_{0}^{-}=0,
\end{aligned}
$$

e podemos provar então que ambos são ortogonais ao vetor $\varepsilon_{\mathrm{r}} \boldsymbol{k}$. De fato, usando a equação (3.63), as equações (3.75) e (3.76) podem ser escritas na forma

$$
\frac{\omega_{ \pm}^{2}}{c^{2}} \varepsilon_{\mathrm{r}} \boldsymbol{E}_{0}^{ \pm}=-\sigma_{D_{E}}^{\prime}(\boldsymbol{k}) \boldsymbol{E}_{0}^{ \pm}
$$

Tomando o produto escalar com $\boldsymbol{k}$, usando que as matrizes $\varepsilon_{\mathrm{r}}$ e $\sigma_{D_{E}}^{\prime}(\boldsymbol{k})$ são simétricas e aplicando a equação (3.65), obtemos

$$
\frac{\omega_{ \pm}^{2}}{c^{2}} \varepsilon_{\mathrm{r}} \boldsymbol{k} \cdot \boldsymbol{E}_{0}^{ \pm}=\frac{\omega_{ \pm}^{2}}{c^{2}} \boldsymbol{k} \cdot \varepsilon_{\mathrm{r}} \boldsymbol{E}_{0}^{ \pm}=-\boldsymbol{k} \cdot \sigma_{D_{E}}^{\prime}(\boldsymbol{k}) \boldsymbol{E}_{0}^{ \pm}=-\sigma_{D_{E}}^{\prime}(\boldsymbol{k}) \boldsymbol{k} \cdot \boldsymbol{E}_{0}^{ \pm}=0
$$

Isso implica que os vetores $\boldsymbol{E}_{0}^{+}$e $\boldsymbol{E}_{0}^{-}$também satisfazem as equações

$$
\begin{aligned}
& \sigma_{D_{E}}\left(\omega_{+}, \boldsymbol{k}, \lambda\right) \boldsymbol{E}_{0}^{+}=0, \\
& \sigma_{D_{E}}\left(\omega_{-}, \boldsymbol{k}, \lambda\right) \boldsymbol{E}_{0}^{-}=0,
\end{aligned}
$$

para qualquer valor de $\lambda$. Além disso, os vetores $\boldsymbol{E}_{0}^{+}$e $\boldsymbol{E}_{0}^{-}$são ortogonais entre si "módulo $\varepsilon_{\mathrm{r}}$ ", pois

$$
\begin{aligned}
\left(\frac{\omega_{+}^{2}}{c^{2}}-\frac{\omega_{-}^{2}}{c^{2}}\right) \boldsymbol{E}_{0}^{+} \varepsilon_{\mathrm{r}} \boldsymbol{E}_{0}^{-} & =\frac{\omega_{+}^{2}}{c^{2}} \varepsilon_{\mathrm{r}} \boldsymbol{E}_{0}^{+} \cdot \boldsymbol{E}_{0}^{-}-\frac{\omega_{-}^{2}}{c^{2}} \boldsymbol{E}_{0}^{+} \cdot \varepsilon_{\mathrm{r}} \boldsymbol{E}_{0}^{-} \\
& =-\sigma_{D_{E}}^{\prime}(\boldsymbol{k}) \boldsymbol{E}_{0}^{+} \cdot \boldsymbol{E}_{0}^{-}+\boldsymbol{E}_{0}^{+} \cdot \sigma_{D_{E}}^{\prime}(\boldsymbol{k}) \boldsymbol{E}_{0}^{-}=0
\end{aligned}
$$

Em particular, são linearmente independentes.

Quando, por outro lado, fixamos $\omega_{+}$e $\omega_{-}$e perguntamos qual seria a forma do conjunto dos vetores de onda $\boldsymbol{k}$ que satisfazem a equação (3.71) ou a equação (3.72), obtemos dois elipsoides entrelaçados, chamados de superfícies normais, que no caso geral têm quatro pontos de intersecção. Porém, no caso isotrópico, esses dois elipsoides coalescem em uma única superfície esférica. 


\subsection{Formulação covariante}

Começamos revendo algumas fórmulas pertinentes à formulação covariante da eletrodinâmica, extraídas das referências [6, 17].

O procedimento básico consiste em aglomerar todas as quantidades físicas importantes na forma de quadrivetores, que possuem uma versão contravariante (índice superior) e uma versão covariante (índice inferior), e de quadritensores, que além destas duas ainda podem possuir versões mistas, sendo que o prefixo "quadri" é frequentemente omitido para não sobrecarregar a terminologia. ${ }^{4} \mathrm{O}$ quadrivetor mais básico é o de posição no espaçotempo:

$$
x^{\mu}=\left(x^{0}, \boldsymbol{x}\right)=(c t, \boldsymbol{x}), x_{\mu}=\left(x_{0},-\boldsymbol{x}\right)=(c t,-\boldsymbol{x}),
$$

enquanto que a convenção para derivadas parciais e vetores de onda é

$$
\partial_{\mu}=\frac{\partial}{\partial x^{\mu}}=\left(\frac{1}{c} \frac{\partial}{\partial t}, \nabla\right), \partial^{\mu}=\frac{\partial}{\partial x_{\mu}}=\left(\frac{1}{c} \frac{\partial}{\partial t},-\nabla\right)
$$

$\mathrm{e}$

$$
k_{\mu}=\left(k_{0}, \boldsymbol{k}\right)=(\omega / c, \boldsymbol{k}), k^{\mu}=\left(k^{0},-\boldsymbol{k}\right)=(\omega / c,-\boldsymbol{k}) .
$$

Passando para campos, a densidade de carga $\rho$ e densidade de corrente usual $\boldsymbol{j}$ se juntam num campo de quadrivetores chamado simplesmente de densidade de corrente, conforme

$$
\begin{gathered}
j^{\mu}=\left(j^{0}, \boldsymbol{j}\right)=(c \rho, \boldsymbol{j}), \\
j_{\mu}=\left(j_{0},-\boldsymbol{j}\right)=(c \rho,-\boldsymbol{j}),
\end{gathered}
$$

o que permite escrever a lei da conservação da carga (equação (3.8)) em forma covariante:

$$
\partial_{\mu} j^{\mu}=0
$$

No caso do eletromagnetismo em meios, o mesmo procedimento é aplicado separadamente às partes externas e internas, ou seja,

$$
\begin{gathered}
j_{\text {ext }}^{\mu}=\left(j_{\text {ext }}^{0}, \boldsymbol{j}_{\text {ext }}\right)=\left(c \rho_{\text {ext }}, \boldsymbol{j}_{\text {ext }}\right), \\
\left(j_{\text {ext }}\right)_{\mu}=\left(j_{\text {ext }, 0},-j_{\text {ext }}\right)=\left(c \rho_{\text {ext }},-\boldsymbol{j}_{\text {ext }}\right),
\end{gathered}
$$

$\mathrm{e}$

$$
\begin{gathered}
j_{\text {mat }}^{\mu}=\left(j_{\text {mat }}^{0}, \boldsymbol{j}_{\text {mat }}\right)=\left(c \rho_{\text {mat }}, \boldsymbol{j}_{\text {mat }}\right), \\
\left(j_{\text {mat }}\right)_{\mu}=\left(j_{\text {mat }, 0},-\boldsymbol{j}_{\text {mat }}\right)=\left(c \rho_{\text {mat }},-\boldsymbol{j}_{\text {mat }}\right),
\end{gathered}
$$

\footnotetext{
${ }^{4}$ Nossa convenção para a métrica no espaço de Minkowski $\mathbb{R}^{4}$ é + - - - e seguimos sistematicamente a convenção da soma de Einstein.
} 
de modo que as equações (3.11) e (3.12) se juntam em

$$
j=j_{\text {ext }}+j_{\text {mat }},
$$

e a condição de conservação separada (equações (3.13) e (3.14)) fica

$$
\begin{gathered}
\partial_{\mu} j_{\text {ext }}^{\mu}=0 . \\
\partial_{\mu} j_{\text {mat }}^{\mu}=0 .
\end{gathered}
$$

De modo análogo, o potencial escalar $\phi$ e o potencial vetorial usual $\boldsymbol{A}$ se juntam num campo de quadrivetores chamado simplesmente o potencial (eletromagnético), conforme

$$
A^{\mu}=\left(A^{0}, \boldsymbol{A}\right)=(\phi / \varkappa c, \boldsymbol{A}), A_{\mu}=\left(A_{0},-\boldsymbol{A}\right)=(\phi / \varkappa c,-\boldsymbol{A}) .
$$

Os campos são obtidos dos potenciais por diferenciação: O campo elétrico $\boldsymbol{E}$ e o campo magnético $\boldsymbol{B}$ são reunidos num campo de quadritensores antissimétrico de segunda ordem chamado simplesmente o campo (eletromagnético), conforme

$$
\begin{aligned}
& -F^{0 i}=F_{0 i}=E_{i} / \varkappa c, \\
& F^{i j}=F_{i j}=-\epsilon_{i j k} B_{k},
\end{aligned}
$$

ou em forma matricial,

$$
\begin{aligned}
F^{\mu \nu} & =\left(\begin{array}{cccc}
0 & -E_{1} / \varkappa c & -E_{2} / \varkappa c & -E_{3} / \varkappa c \\
+E_{1} / \varkappa c & 0 & -B_{3} & +B_{2} \\
+E_{2} / \varkappa c & +B_{3} & 0 & -B_{1} \\
+E_{3} / \varkappa c & -B_{2} & +B_{1} & 0
\end{array}\right), \\
F_{\mu \nu} & =\left(\begin{array}{cccc}
0 & +E_{1} / \varkappa c & +E_{2} / \varkappa c & +E_{3} / \varkappa c \\
-E_{1} / \varkappa c & 0 & -B_{3} & +B_{2} \\
-E_{2} / \varkappa c & +B_{3} & 0 & -B_{1} \\
-E_{3} / \varkappa c & -B_{2} & +B_{1} & 0
\end{array}\right),
\end{aligned}
$$

o que permite escrever a definição dos campos em termos dos potenciais (equações (3.36) e (3.37)) em forma covariante,

$$
F_{\mu \nu}=\partial_{\mu} A_{\nu}-\partial_{\nu} A_{\mu}
$$

e as equações homogêneas de Maxwell (3.15-b) e (3.15-c) em forma covariante,

$$
\partial_{\kappa} F_{\mu \nu}+\partial_{\mu} F_{\nu \kappa}+\partial_{\nu} F_{\kappa \mu}=0
$$


No caso do eletromagnetismo em meios, o mesmo procedimento é aplicado aos campos $\boldsymbol{D}$ e $\boldsymbol{H}$, i.e.,

$$
\begin{gathered}
-G^{0 i}=G_{0 i}=D_{i} / \varkappa \varepsilon_{0} c, \\
G^{i j}=G_{i j}=-\mu_{0} \epsilon_{i j k} H_{k},
\end{gathered}
$$

ou em forma matricial,

$$
\begin{aligned}
G^{\mu \nu}= & \left(\begin{array}{cccc}
0 & -D_{1} / \varkappa \varepsilon_{0} c & -D_{2} / \varkappa \varepsilon_{0} c & -D_{3} / \varkappa \varepsilon_{0} c \\
+D_{1} / \varkappa \varepsilon_{0} c & 0 & -\mu_{0} H_{3} & +\mu_{0} H_{2} \\
+D_{2} / \varkappa \varepsilon_{0} c & +\mu_{0} H_{3} & 0 & -\mu_{0} H_{1} \\
+D_{3} / \varkappa \varepsilon_{0} c & -\mu_{0} H_{2} & +\mu_{0} H_{1} & 0
\end{array}\right), \\
G_{\mu \nu}= & \left(\begin{array}{cccc}
0 & +D_{1} / \varkappa \varepsilon_{0} c & +D_{2} / \varkappa \varepsilon_{0} c & +D_{3} / \varkappa \varepsilon_{0} c \\
-D_{1} / \varkappa \varepsilon_{0} c & 0 & -\mu_{0} H_{3} & +\mu_{0} H_{2} \\
-D_{2} / \varkappa \varepsilon_{0} c & +\mu_{0} H_{3} & 0 & -\mu_{0} H_{1} \\
-D_{3} / \varkappa \varepsilon_{0} c & -\mu_{0} H_{2} & +\mu_{0} H_{1} & 0
\end{array}\right),
\end{aligned}
$$

o que permite escrever as equações não homogêneas de Maxwell (3.15-a) e (3.15-d) em forma covariante,

$$
\partial_{\mu} G^{\mu \nu}=\varkappa \mu_{0} j_{\mathrm{ext}}^{\nu} .
$$

Notemos ainda que as relações constitutivas (3.1), (3.2) também podem ser reescritas em forma covariante. Para tanto, introduzimos tensores $\Lambda^{(1)} \mathrm{e}$ $\Lambda^{(2)}$ que representam, respectivamente, transformações lineares no espaço $\bigwedge^{1} \mathbb{R}^{4} \cong \mathbb{R}^{4}$ e no espaço $\bigwedge^{2} \mathbb{R}^{4} \cong \mathbb{R}^{6}$, sendo que, por abuso de notação, denotaremos ambos simplesmente por $\Lambda$ (distinguindo-os pelas respectivas estruturas de índices). Explicitamente, $\Lambda^{(1)}$ é dado por

$$
\begin{gathered}
\Lambda^{0,0}=\Lambda_{0,0}=\Lambda_{0}^{0}=1, \\
-\Lambda^{i, k}=-\Lambda_{i, k}=\Lambda_{i}^{k}=\left(\varepsilon_{\mathrm{r}}\right)_{i k},
\end{gathered}
$$

enquanto que $\Lambda^{(2)}$ é dado por

$$
\begin{gathered}
-\Lambda^{0 i, 0 k}=-\Lambda_{0 i, 0 k}=\Lambda_{0 i}^{0 k}=\left(\varepsilon_{\mathrm{r}}\right)_{i k}, \\
\Lambda^{i j, k l}=\Lambda_{i j, k l}=\Lambda_{i j}^{k l}=\epsilon_{i j p}\left(\mu_{\mathrm{r}}^{-1}\right)_{p q} \epsilon_{q k l},
\end{gathered}
$$

ou ainda, devido à relação (3.29),

$$
\begin{gathered}
-\Lambda^{0 i, 0 k}=-\Lambda_{0 i, 0 k}=\Lambda_{0 i}^{0 k}=\left(\varepsilon_{\mathrm{r}}\right)_{i k} \\
\Lambda^{i j, k l}=\Lambda_{i j, k l}=\Lambda_{i j}^{k l}=\frac{1}{\operatorname{det} \mu_{\mathrm{r}}}\left(\left(\mu_{\mathrm{r}}\right)_{i k}\left(\mu_{\mathrm{r}}\right)_{j l}-\left(\mu_{\mathrm{r}}\right)_{i l}\left(\mu_{\mathrm{r}}\right)_{j k}\right) .
\end{gathered}
$$


Ambos satisfazem condições de simetria, a saber,

$$
\begin{gathered}
\Lambda_{\mu, \kappa}=\Lambda_{\kappa, \mu}, \\
\Lambda_{\lambda \kappa, \mu \nu}=-\Lambda_{\kappa \lambda, \mu \nu}=\Lambda_{\kappa \lambda, \nu \mu} \quad, \quad \Lambda_{\mu \nu, \kappa \lambda}=\Lambda_{\kappa \lambda, \mu \nu},
\end{gathered}
$$

as quais expressam a simetria de $\varepsilon_{\mathrm{r}}$ e $\mu_{\mathrm{r}}$; relações análogas valem para os respectivos inversos. Com isso, segue

$$
G_{\mu \nu}=\frac{1}{2} \Lambda_{\mu \nu}^{\kappa \lambda} F_{\kappa \lambda} \quad, \quad G^{\mu \nu}=\frac{1}{2} \Lambda_{\kappa \lambda}^{\mu \nu} F^{\kappa \lambda},
$$

e definimos $\Lambda A$ e $\Lambda^{-1} j$ por

$$
(\Lambda A)_{\mu}=\Lambda_{\mu}^{\nu} A_{\nu} \quad, \quad\left(\Lambda^{-1} j_{\text {ext }}\right)_{\mu}=\left(\Lambda^{-1}\right)_{\mu}^{\nu} j_{\text {ext }, \nu}
$$

ou seja,

$$
\begin{gathered}
(\Lambda A)_{\mu}=\left(A_{0},-\varepsilon_{\mathrm{r}} \boldsymbol{A}\right)=\left(\phi / \varkappa c,-\varepsilon_{\mathrm{r}} \boldsymbol{A}\right) . \\
\left(\Lambda^{-1} j_{\text {ext }}\right)_{\mu}=\left(j_{\text {ext }, 0},-\varepsilon_{\mathrm{r}}^{-1} \boldsymbol{j}_{\text {ext }}\right)=\left(c \rho_{\text {ext }},-\varepsilon_{\mathrm{r}}^{-1} \boldsymbol{j}_{\text {ext }}\right) .
\end{gathered}
$$

Com essas notações, veremos agora como expressar as diversas equações de onda que apareceram nas seções anteriores deste capítulo também em forma covariante.

Começamos pelos potenciais, que é um caso mais simples. Para tanto, notamos inicialmente que o a condição de calibre de Lorenz modificado da equação (3.40) pode ser escrita em forma covariante em termos do tensor $\Lambda^{(1)}$ :

$$
\Lambda^{\mu, \nu} \partial_{\mu} A_{\nu}=0
$$

Então usando as equações (3.96), (3.101) e (3.92), obtemos

$$
\begin{aligned}
\varkappa \mu_{0} j_{\text {ext }, \rho} & =\eta^{\mu \nu} \partial_{\mu} G_{\nu \rho}=\frac{1}{2} \eta^{\mu \nu} \Lambda_{\nu \rho}^{\kappa \lambda} \partial_{\mu} F_{\kappa \lambda} \\
& =\frac{1}{2} \eta^{\mu \nu} \Lambda_{\nu \rho}^{\kappa \lambda}\left(\partial_{\mu} \partial_{\kappa} A_{\lambda}-\partial_{\mu} \partial_{\lambda} A_{\kappa}\right) \\
& =\eta^{\mu \nu} \Lambda_{\nu \rho}^{\kappa \lambda} \partial_{\mu} \partial_{\kappa} A_{\lambda} \\
& =\eta^{\mu \lambda} \Lambda_{\lambda \rho}^{\nu \kappa} \partial_{\mu} \partial_{\nu} A_{\kappa}+\Lambda_{\rho}^{\mu} \Lambda^{\nu, \kappa} \partial_{\mu} \partial_{\nu} A_{\kappa},
\end{aligned}
$$

onde, no último passo, adicionamos um termo adequadamente escolhido que se anula devido à equação (3.104). Isso nos dá a equação de onda para o potencial eletromagnético $A$ na sua forma covariante, no calibre de Lorenz modificado,

$$
\left(\sigma_{A}^{\mu \nu}\right)_{\rho}^{\kappa} \partial_{\mu} \partial_{\nu} A_{\kappa}=\varkappa \mu_{0} j_{\text {ext }, \rho}
$$


onde, mediante simetrização em $\mu$ e $\nu$,

$$
\left(\sigma_{A}^{\mu \nu}\right)_{\rho}^{\kappa}=\frac{1}{2}\left(\eta^{\mu \lambda} \Lambda_{\lambda \rho}^{\nu \kappa}+\eta^{\nu \lambda} \Lambda_{\lambda \rho}^{\mu \kappa}+\Lambda_{\rho}^{\mu} \Lambda^{\nu, \kappa}+\Lambda_{\rho}^{\nu} \Lambda^{\mu, \kappa}\right) .
$$

Para entender melhor a estrutura deste tensor, calculamos explicitamente a contração $\left(\sigma_{A}^{\mu \nu}\right)_{\rho}^{\kappa} \xi_{\mu} \xi_{\nu} a_{\kappa}$ para vetores $\xi$ e $a$ em $\mathbb{R}^{4}$. No caso $\rho=0$, esta expressão vale

$$
\begin{aligned}
- & \Lambda_{l 0}^{n 0} \xi_{l} \xi_{n} a_{0}-\Lambda_{l 0}^{0 k} \xi_{l} \xi_{0} a_{k}+\xi_{0} \xi_{0} a_{0}+\Lambda^{n, k} \xi_{0} \xi_{n} a_{k} \\
& =-\left(\varepsilon_{\mathrm{r}}\right)_{l n} \xi_{l} \xi_{n} a_{0}+\left(\varepsilon_{\mathrm{r}}\right)_{l k} \xi_{l} \xi_{0} a_{k}+\xi_{0} \xi_{0} a_{0}-\left(\varepsilon_{\mathrm{r}}\right)_{n k} \xi_{0} \xi_{n} a_{k} \\
& =\Lambda^{\mu, \nu} \xi_{\mu} \xi_{\nu} a_{0},
\end{aligned}
$$

enquanto que no caso $\rho=r$, ela vale

$$
\begin{aligned}
\Lambda_{0 r}^{n 0} \xi_{0} & \xi_{n} a_{0}+\Lambda_{0 r}^{0 k} \xi_{0} \xi_{0} a_{k}-\Lambda_{l r}^{n k} \xi_{l} \xi_{n} a_{k}+\Lambda_{r}^{m} \xi_{m} \xi_{0} a_{0}+\Lambda_{r}^{m} \Lambda^{n, k} \xi_{m} \xi_{n} a_{k} \\
= & -\left(\varepsilon_{\mathrm{r}}\right)_{r n} \xi_{0} \xi_{n} a_{0}+\left(\varepsilon_{\mathrm{r}}\right)_{r k} \xi_{0} \xi_{0} a_{k}-\epsilon_{l r p}\left(\mu_{\mathrm{r}}^{-1}\right)_{p q} \epsilon_{q n k} \xi_{l} \xi_{n} a_{k} \\
& \quad+\left(\varepsilon_{\mathrm{r}}\right)_{r m} \xi_{m} \xi_{0} a_{0}-\left(\varepsilon_{\mathrm{r}}\right)_{r m}\left(\varepsilon_{\mathrm{r}}\right)_{n k} \xi_{m} \xi_{n} a_{k} \\
= & \Lambda^{\mu, \nu}\left(\varepsilon_{\mathrm{r}}\right)_{r k} \xi_{\mu} \xi_{\nu} a_{k}+\left(\varepsilon_{\mathrm{r}}\right)_{i j}\left(\varepsilon_{\mathrm{r}}\right)_{r k} \xi_{i} \xi_{j} a_{k}-\left(\varepsilon_{\mathrm{r}}\right)_{r i}\left(\varepsilon_{\mathrm{r}}\right)_{j k} \xi_{i} \xi_{j} a_{k} \\
& \quad+\epsilon_{r i p}\left(\mu_{\mathrm{r}}^{-1}\right)_{p q} \epsilon_{q j k} \xi_{i} \xi_{j} a_{k} .
\end{aligned}
$$

Então introduzindo o tensor puramente espacial dado por

$$
\begin{aligned}
\left(\Lambda_{\mathrm{sp}}^{i j}\right)_{r s}= & \left(\varepsilon_{\mathrm{r}}\right)_{i j}\left(\varepsilon_{\mathrm{r}}\right)_{r s}-\frac{1}{2}\left(\varepsilon_{\mathrm{r}}\right)_{i r}\left(\varepsilon_{\mathrm{r}}\right)_{j s}-\frac{1}{2}\left(\varepsilon_{\mathrm{r}}\right)_{j r}\left(\varepsilon_{\mathrm{r}}\right)_{i s} \\
& -\frac{1}{\operatorname{det} \mu_{\mathrm{r}}}\left(\left(\mu_{\mathrm{r}}\right)_{i j}\left(\mu_{\mathrm{r}}\right)_{r s}-\frac{1}{2}\left(\mu_{\mathrm{r}}\right)_{i r}\left(\mu_{\mathrm{r}}\right)_{j s}-\frac{1}{2}\left(\mu_{\mathrm{r}}\right)_{j r}\left(\mu_{\mathrm{r}}\right)_{i s}\right)
\end{aligned}
$$

$\left(\operatorname{com}\left(\Lambda_{\mathrm{sp}}^{i j}\right)_{r}^{k}=-\left(\Lambda_{\mathrm{sp}}^{i j}\right)_{r k}\right.$ e $\left(\Lambda_{\mathrm{sp}}^{\mu \nu}\right)_{\rho}^{\kappa}=0$ se qualquer um dos índices $\mu, \nu, \kappa, \rho$ for igual a 0$)$, concluímos que

$$
\left(\sigma_{A}^{\mu \nu}\right)_{\rho}^{\kappa}=\Lambda^{\mu, \nu} \Lambda_{\rho}^{\kappa}+\left(\Lambda_{\mathrm{sp}}^{\mu \nu}\right)_{\rho}^{\kappa} .
$$

Com este resultado, fica claro que a componente temporal e as componentes espaciais da equação (3.105) reproduzem, respectivamente, as equações (3.41) e (3.42).

Para os campos, tentamos de proceder de forma análoga; porém, aqui os cálculos são bem mais complexos. Primeiro, aplicando o inverso do tensor $\Lambda^{(1)}$ à equação (3.96), tomando a derivada exterior e usando a equação (3.101), obtemos

$$
\begin{aligned}
& \varkappa \mu_{0}\left(\partial_{\rho}\left(\Lambda^{-1} j_{\text {ext }}\right)_{\sigma}-\partial_{\sigma}\left(\Lambda^{-1} j_{\text {ext }}\right)_{\rho}\right) \\
& =\eta^{\mu \alpha}\left(\Lambda^{-1}\right)_{\sigma}^{\beta} \partial_{\rho} \partial_{\mu} G_{\alpha \beta}-\eta^{\mu \alpha}\left(\Lambda^{-1}\right)_{\rho}^{\beta} \partial_{\sigma} \partial_{\mu} G_{\alpha \beta} \\
& =\frac{1}{2} \eta^{\mu \alpha}\left(\Lambda^{-1}\right)_{\sigma}^{\beta} \Lambda_{\alpha \beta}^{\kappa \lambda} \partial_{\mu} \partial_{\rho} F_{\kappa \lambda}-\frac{1}{2} \eta^{\mu \alpha}\left(\Lambda^{-1}\right)_{\rho}^{\beta} \Lambda_{\alpha \beta}^{\kappa \lambda} \partial_{\mu} \partial_{\sigma} F_{\kappa \lambda} .
\end{aligned}
$$


No caso do eletromagnetismo no vácuo, onde vale $\varepsilon_{\mathrm{r}}=1, \mu_{\mathrm{r}}=1$ e portanto temos $\Lambda_{\rho}^{\kappa}=\delta_{\rho}^{\kappa}, \Lambda_{\rho \sigma}^{\kappa \lambda}=\delta_{\rho}^{\kappa} \delta_{\sigma}^{\lambda}-\delta_{\sigma}^{\kappa} \delta_{\rho}^{\lambda}$ e $G=F$, esta expressão se reduz a $\eta^{\mu \nu} \partial_{\mu}\left(\partial_{\rho} F_{\nu \sigma}-\partial_{\sigma} F_{\nu \rho}\right)$, o que pela equação homogênea (3.93) é igual a $\eta^{\mu \nu} \partial_{\mu} \partial_{\nu} F_{\rho \sigma}$, e este já é o resultado final. Mas no caso do eletromagnetismo em meios, precisamos fazer uso da equação homogênea (3.93) de forma muito mais refinada, e foi aqui que enfrentamos o problema mais difícil de todo este trabalho, uma vez que nossa meta foi obter um sistema de equações de onda para os campos com propriedades matemáticas satisfatórias, a saber,

- As equações para os campos do tipo elétrico $\left(F_{0 k}\right.$ e $G_{0 k}$, ou seja, $\boldsymbol{E}$ e $\left.\boldsymbol{D}\right)$ e os campos do tipo magnético $\left(F_{k l}\right.$ e $G_{k l}$, ou seja, $\boldsymbol{B}$ e $\left.\boldsymbol{H}\right)$ desacoplam.

- Não aparecem segundas derivadas mistas to tipo $\partial_{0} \partial_{i}$.

Para atingir esta meta, parece ser imprescindível complementar a expressão acima por uma combinação não covariante da componente temporal e das componentes espaciais da expressão que se anula devido à equação homogênea (3.93). Mais especificamente, note que, devido à equação homogênea (3.93), vale

$$
\partial_{\tau} F_{\kappa \lambda}=\partial_{\kappa} F_{\tau \lambda}-\partial_{\lambda} F_{\tau \kappa}
$$

e também

$$
\partial_{\tau} F_{\kappa \lambda}=\frac{1}{2}\left(\partial_{\tau} F_{\kappa \lambda}+\partial_{\kappa} F_{\tau \lambda}-\partial_{\lambda} F_{\tau \kappa}\right)
$$

Então aplicaremos a seguinte regra: quando $\rho=0$ e $\sigma=s$, substituímos $\partial_{\rho} F_{\kappa \lambda}$ por $\partial_{\kappa} F_{\rho \lambda}-\partial_{\lambda} F_{\rho \kappa}$ mas não fazemos nenhuma substituição de $\partial_{\sigma} F_{\kappa \lambda}$, enquanto que quando $\rho=r$ e $\sigma=s$, substituímos tanto $\partial_{\rho} F_{\kappa \lambda}$ como $\partial_{\sigma} F_{\kappa \lambda}$ pela combinação do lado direito da segunda expressão acima (com $\tau$ substituído por $\rho$ e por $\sigma$, respectivamente). Com isso, obtemos para $\rho=0$ e $\sigma=s$,

$$
\begin{aligned}
\varkappa \mu_{0} & \left(\partial_{0}\left(\Lambda^{-1} j_{\text {ext }}\right)_{s}-\partial_{s} j_{\text {ext }, 0}\right) \\
= & \frac{1}{2} \eta^{\mu \alpha}\left(\Lambda^{-1}\right)_{s}^{b} \Lambda_{\alpha b}^{\kappa \lambda} \partial_{\mu}\left(\partial_{\kappa} F_{0 \lambda}-\partial_{\lambda} F_{0 \kappa}\right)-\frac{1}{2} \eta^{\mu \alpha} \Lambda_{\alpha 0}^{\kappa \lambda} \partial_{\mu} \partial_{s} F_{\kappa \lambda} \\
= & \eta^{\mu \alpha}\left(\Lambda^{-1}\right)_{s}^{b} \Lambda_{\alpha b}^{\kappa \lambda} \partial_{\mu} \partial_{\kappa} F_{0 \lambda}-\frac{1}{2} \eta^{\mu \alpha} \Lambda_{\alpha 0}^{\kappa \lambda} \partial_{\mu} \partial_{s} F_{\kappa \lambda} \\
= & \left(\Lambda^{-1}\right)_{s}^{b} \Lambda_{0 b}^{0 l} \partial_{0} \partial_{0} F_{0 l}-\left(\Lambda^{-1}\right)_{s}^{b} \Lambda_{i b}^{k l} \partial_{i} \partial_{k} F_{0 l} \\
& \quad+\frac{1}{2} \Lambda_{i 0}^{0 l} \partial_{i} \partial_{s} F_{0 l}+\frac{1}{2} \Lambda_{i 0}^{k 0} \partial_{i} \partial_{s} F_{k 0} \\
= & \partial_{0} \partial_{0} F_{0 s}-\partial_{s}\left(\left(\varepsilon_{\mathrm{r}}\right)_{k l} \partial_{k} F_{0 l}\right)-\left(\varepsilon_{\mathrm{r}}^{-1}\right)_{s t} \epsilon_{t i p}\left(\mu_{\mathrm{r}}^{-1}\right)_{p q} \epsilon_{q k l} \partial_{i} \partial_{k} F_{0 l},
\end{aligned}
$$


o que coincide com a equação de onda (3.20) para o campo elétrico, enquanto que, para $\rho=r$ e $\sigma=s$,

$$
\begin{aligned}
\varkappa \mu_{0}( & \left.\partial_{r}\left(\Lambda^{-1} j_{\mathrm{ext}}\right)_{s}-\partial_{s}\left(\Lambda^{-1} j_{\mathrm{ext}}\right)_{r}\right) \\
=\frac{1}{4} & \eta^{\mu \alpha}\left(\Lambda^{-1}\right)_{s}^{b} \Lambda_{\alpha b}^{\kappa \lambda} \partial_{\mu}\left(\partial_{r} F_{\kappa \lambda}+\partial_{\kappa} F_{r \lambda}-\partial_{\lambda} F_{r \kappa}\right) \\
& -\frac{1}{4} \eta^{\mu \alpha}\left(\Lambda^{-1}\right)_{r}^{b} \Lambda_{\alpha b}^{\kappa \lambda} \partial_{\mu}\left(\partial_{s} F_{\kappa \lambda}+\partial_{\kappa} F_{s \lambda}-\partial_{\lambda} F_{s \kappa}\right) \\
=\frac{1}{4} & \left(\Lambda^{-1}\right)_{s}^{b} \Lambda_{0 b}^{0 l} \partial_{0}\left(\partial_{r} F_{0 l}+\partial_{0} F_{r l}-\partial_{l} F_{r 0}\right) \\
& +\frac{1}{4}\left(\Lambda^{-1}\right)_{s}^{b} \Lambda_{0 b}^{k 0} \partial_{0}\left(\partial_{r} F_{k 0}+\partial_{k} F_{r 0}-\partial_{0} F_{r k}\right) \\
& -\frac{1}{4}\left(\Lambda^{-1}\right)_{s}^{b} \Lambda_{i b}^{k l} \partial_{i}\left(\partial_{r} F_{k l}+\partial_{k} F_{r l}-\partial_{l} F_{r k}\right) \\
& -\frac{1}{4}\left(\Lambda^{-1}\right)_{r}^{b} \Lambda_{0 b}^{0 l} \partial_{0}\left(\partial_{s} F_{0 l}+\partial_{0} F_{s l}-\partial_{l} F_{s 0}\right) \\
& -\frac{1}{4}\left(\Lambda^{-1}\right)_{r}^{b} \Lambda_{0 b}^{k 0} \partial_{0}\left(\partial_{s} F_{k 0}+\partial_{k} F_{s 0}-\partial_{0} F_{s k}\right) \\
& +\frac{1}{4}\left(\Lambda^{-1}\right)_{r}^{b} \Lambda_{i b}^{k l} \partial_{i}\left(\partial_{s} F_{k l}+\partial_{k} F_{s l}-\partial_{l} F_{s k}\right) \\
=\frac{1}{4} & \partial_{0}\left(\partial_{r} F_{0 s}+\partial_{0} F_{r s}-\partial_{s} F_{r 0}\right) \\
& -\frac{1}{4} \partial_{0}\left(\partial_{r} F_{s 0}+\partial_{s} F_{r 0}-\partial_{0} F_{r s}\right) \\
& -\frac{1}{4} \partial_{0}\left(\partial_{s} F_{0 r}+\partial_{0} F_{s r}-\partial_{r} F_{s 0}\right) \\
& +\frac{1}{4} \partial_{0}\left(\partial_{s} F_{r 0}+\partial_{r} F_{s 0}-\partial_{0} F_{s r}\right) \\
& +\frac{1}{4}\left(\varepsilon_{\mathrm{r}}^{-1}\right)_{s t} \epsilon_{t i p}\left(\mu_{\mathrm{r}}^{-1}\right)_{p q} \epsilon_{q k l} \partial_{i}\left(\partial_{r} F_{k l}+\partial_{k} F_{r l}-\partial_{l} F_{r k}\right) \\
& -\frac{1}{4}\left(\varepsilon_{\mathrm{r}}^{-1}\right)_{r t} \epsilon_{t i p}\left(\mu_{\mathrm{r}}^{-1}\right)_{p q} \epsilon_{q k l} \partial_{i}\left(\partial_{s} F_{k l}+\partial_{k} F_{s l}-\partial_{l} F_{s k}\right) \\
= & \partial_{0} F_{r s}-
\end{aligned}
$$

\section{VERSÃO 2:}

Primeiro, aplicando o inverso de $\Lambda^{(1)}$ à equação (3.96), tomando a derivada exterior e usando a equação (3.101), obtemos

$$
\begin{aligned}
\varkappa & \mu_{0}\left(\partial_{\rho}\left(\Lambda^{-1} j_{\text {ext }}\right)_{\sigma}-\partial_{\sigma}\left(\Lambda^{-1} j_{\text {ext }}\right)_{\rho}\right) \\
= & \eta^{\mu \alpha}\left(\Lambda^{-1}\right)_{\sigma}^{\beta} \partial_{\rho} \partial_{\mu} G_{\alpha \beta}-\eta^{\mu \alpha}\left(\Lambda^{-1}\right)_{\rho}^{\beta} \partial_{\sigma} \partial_{\mu} G_{\alpha \beta} \\
= & \frac{1}{2} \eta^{\mu \alpha}\left(\Lambda^{-1}\right)_{\sigma}^{\beta} \Lambda_{\alpha \beta}^{\kappa \lambda} \partial_{\rho} \partial_{\mu} F_{\kappa \lambda}-\frac{1}{2} \eta^{\mu \alpha}\left(\Lambda^{-1}\right)_{\rho}^{\beta} \Lambda_{\alpha \beta}^{\kappa \lambda} \partial_{\sigma} \partial_{\mu} F_{\kappa \lambda} \\
& -\frac{1}{4} \eta^{\mu \alpha}\left(\Lambda^{-1}\right)_{\sigma}^{\beta} \Lambda_{\alpha \beta}^{\kappa \lambda} \partial_{\mu}\left(\partial_{\rho} F_{\kappa \lambda}-\partial_{\kappa} F_{\rho \lambda}+\partial_{\lambda} F_{\rho \kappa}\right) \\
& +\frac{1}{4} \eta^{\mu \alpha}\left(\Lambda^{-1}\right)_{\rho}^{\beta} \Lambda_{\alpha \beta}^{\kappa \lambda} \partial_{\mu}\left(\partial_{\sigma} F_{\kappa \lambda}-\partial_{\kappa} F_{\sigma \lambda}+\partial_{\lambda} F_{\sigma \kappa}\right) \\
= & \frac{1}{2}\left(\eta^{\mu \alpha} \delta_{\rho}^{\nu}\left(\Lambda^{-1}\right)_{\sigma}^{\beta} \Lambda_{\alpha \beta}^{\kappa \lambda}-\eta^{\mu \alpha} \delta_{\sigma}^{\nu}\left(\Lambda^{-1}\right)_{\rho}^{\beta} \Lambda_{\alpha \beta}^{\kappa \lambda}\right) \partial_{\mu} \partial_{\nu} F_{\kappa \lambda},
\end{aligned}
$$


onde ainda falta adicionar um termo adequadamente escolhido que se anula devido à equação (3.93); aqui estão duas possibilidades que merecem ser investigadas:

VERSÃO 2a:

$$
\begin{aligned}
\varkappa \mu_{0} & \left(\partial_{\rho}\left(\Lambda^{-1} j_{\text {ext }}\right)_{\sigma}-\partial_{\sigma}\left(\Lambda^{-1} j_{\text {ext }}\right)_{\rho}\right) \\
= & \eta^{\mu \alpha}\left(\Lambda^{-1}\right)_{\sigma}^{\beta} \partial_{\rho} \partial_{\mu} G_{\alpha \beta}-\eta^{\mu \alpha}\left(\Lambda^{-1}\right)_{\rho}^{\beta} \partial_{\sigma} \partial_{\mu} G_{\alpha \beta} \\
= & \frac{1}{2} \eta^{\mu \alpha}\left(\Lambda^{-1}\right)_{\sigma}^{\beta} \Lambda_{\alpha \beta}^{\kappa \lambda} \partial_{\rho} \partial_{\mu} F_{\kappa \lambda}-\frac{1}{2} \eta^{\mu \alpha}\left(\Lambda^{-1}\right)_{\rho}^{\beta} \Lambda_{\alpha \beta}^{\kappa \lambda} \partial_{\sigma} \partial_{\mu} F_{\kappa \lambda} \\
= & \frac{1}{2} \eta^{\mu \alpha}\left(\Lambda^{-1}\right)_{\sigma}^{\beta} \Lambda_{\alpha \beta}^{\kappa \lambda} \partial_{\mu}\left(\partial_{\kappa} F_{\rho \lambda}-\partial_{\lambda} F_{\rho \kappa}\right) \\
& \quad-\frac{1}{2} \eta^{\mu \alpha}\left(\Lambda^{-1}\right)_{\rho}^{\beta} \Lambda_{\alpha \beta}^{\kappa \lambda} \partial_{\mu}\left(\partial_{\kappa} F_{\sigma \lambda}-\partial_{\lambda} F_{\sigma \kappa}\right) \\
= & \left(\eta^{\mu \alpha} \delta_{\rho}^{\kappa}\left(\Lambda^{-1}\right)_{\sigma}^{\beta} \Lambda_{\alpha \beta}^{\nu \lambda}-\eta^{\mu \alpha} \delta_{\sigma}^{\kappa}\left(\Lambda^{-1}\right)_{\rho}^{\beta} \Lambda_{\alpha \beta}^{\nu \lambda}\right) \partial_{\mu} \partial_{\nu} F_{\kappa \lambda} .
\end{aligned}
$$

VERSÃO 2b:

$$
\begin{aligned}
\varkappa \mu_{0} & \left(\partial_{\rho}\left(\Lambda^{-1} j_{\text {ext }}\right)_{\sigma}-\partial_{\sigma}\left(\Lambda^{-1} j_{\text {ext }}\right)_{\rho}\right) \\
= & \eta^{\mu \alpha}\left(\Lambda^{-1}\right)_{\sigma}^{\beta} \partial_{\rho} \partial_{\mu} G_{\alpha \beta}-\eta^{\mu \alpha}\left(\Lambda^{-1}\right)_{\rho}^{\beta} \partial_{\sigma} \partial_{\mu} G_{\alpha \beta} \\
= & \frac{1}{2} \eta^{\mu \alpha}\left(\Lambda^{-1}\right)_{\sigma}^{\beta} \Lambda_{\alpha \beta}^{\kappa \lambda} \partial_{\rho} \partial_{\mu} F_{\kappa \lambda}-\frac{1}{2} \eta^{\mu \alpha}\left(\Lambda^{-1}\right)_{\rho}^{\beta} \Lambda_{\alpha \beta}^{\kappa \lambda} \partial_{\sigma} \partial_{\mu} F_{\kappa \lambda} \\
= & \frac{1}{4} \eta^{\mu \alpha}\left(\Lambda^{-1}\right)_{\sigma}^{\beta} \Lambda_{\alpha \beta}^{\kappa \lambda} \partial_{\mu}\left(\partial_{\rho} F_{\kappa \lambda}+\partial_{\kappa} F_{\rho \lambda}-\partial_{\lambda} F_{\rho \kappa}\right) \\
& \quad-\frac{1}{4} \eta^{\mu \alpha}\left(\Lambda^{-1}\right)_{\rho}^{\beta} \Lambda_{\alpha \beta}^{\kappa \lambda} \partial_{\mu}\left(\partial_{\sigma} F_{\kappa \lambda}+\partial_{\kappa} F_{\sigma \lambda}-\partial_{\lambda} F_{\sigma \kappa}\right) \\
= & \left(\frac{1}{4} \eta^{\mu \alpha} \delta_{\rho}^{\nu}\left(\Lambda^{-1}\right)_{\sigma}^{\beta} \Lambda_{\alpha \beta}^{\kappa \lambda}-\frac{1}{4} \eta^{\mu \alpha} \delta_{\sigma}^{\nu}\left(\Lambda^{-1}\right)_{\rho}^{\beta} \Lambda_{\alpha \beta}^{\kappa \lambda}\right. \\
& \left.\quad+\frac{1}{2} \eta^{\mu \alpha} \delta_{\rho}^{\kappa}\left(\Lambda^{-1}\right)_{\sigma}^{\beta} \Lambda_{\alpha \beta}^{\nu \lambda}-\frac{1}{2} \eta^{\mu \alpha} \delta_{\sigma}^{\kappa}\left(\Lambda^{-1}\right)_{\rho}^{\beta} \Lambda_{\alpha \beta}^{\nu \lambda}\right) \partial_{\mu} \partial_{\nu} F_{\kappa \lambda} .
\end{aligned}
$$

\section{DE QUALQUER FORMA:}

Isso nos dá a equação de onda para o tensor de campo eletromagnético $F$, na sua forma covariante,

VERSÃO 1:

$$
\left(\sigma_{F}^{\mu \nu}\right)_{\rho \sigma}^{\kappa \lambda} \partial_{\mu} \partial_{\nu} F_{\kappa \lambda}=\varkappa \mu_{0}\left(\partial_{\rho} j_{\mathrm{ext}, \sigma}-\partial_{\sigma} j_{\mathrm{ext}, \rho}\right),
$$

VERSÃO 2:

$$
\left(\sigma_{F}^{\mu \nu}\right)_{\rho \sigma}^{\kappa \lambda} \partial_{\mu} \partial_{\nu} F_{\kappa \lambda}=\varkappa \mu_{0}\left(\partial_{\rho}\left(\Lambda^{-1} j_{\mathrm{ext}}\right)_{\sigma}-\partial_{\sigma}\left(\Lambda^{-1} j_{\mathrm{ext}}\right)_{\rho}\right)
$$

onde resta a determinar o tensor $\sigma_{F}$. 
Para entender melhor a estrutura deste tensor, calculamos explicitamente a contração $\left(\sigma_{F}^{\mu \nu}\right)_{\rho \sigma}^{\kappa \lambda} \xi_{\mu} \xi_{\nu} f_{\kappa \lambda}$ com um vetor $\xi$ em $\mathbb{R}^{4}$ e um tensor antissimétrico $f \mathrm{em} \bigwedge^{2} \mathbb{R}^{4}$.

No caso $\rho=0$ e $\sigma=s$, esta expressão vale

VERSÃO 1:

$$
\begin{aligned}
\frac{1}{2} & \Lambda_{0 s}^{0 l} \xi_{0} \xi_{0} f_{0 l}+\frac{1}{2} \Lambda_{0 s}^{k 0} \xi_{0} \xi_{0} f_{k 0}-\frac{1}{2} \Lambda_{a s}^{k l} \xi_{a} \xi_{0} f_{k l} \\
+ & \frac{1}{2} \Lambda_{a 0}^{0 l} \xi_{a} \xi_{s} f_{0 l}+\frac{1}{2} \Lambda_{a 0}^{k 0} \xi_{a} \xi_{s} f_{k 0} \\
+ & \frac{1}{2} \Lambda_{a s}^{k l} \xi_{a}\left(\xi_{0} f_{k l}-\xi_{k} f_{0 l}+\xi_{l} f_{0 k}\right) \\
= & \left(\varepsilon_{\mathrm{r}}\right)_{s l} \xi_{0} \xi_{0} f_{0 l}-\left(\varepsilon_{\mathrm{r}}\right)_{k l} \xi_{k} \xi_{s} f_{0 l}+\frac{1}{2} \epsilon_{s i p}\left(\mu_{\mathrm{r}}^{-1}\right)_{p q} \epsilon_{q j l} \xi_{i} \xi_{0} f_{j l} \\
& \quad-\frac{1}{2} \epsilon_{s i p}\left(\mu_{\mathrm{r}}^{-1}\right)_{p q} \epsilon_{q j l} \xi_{i}\left(\xi_{0} f_{j l}-\xi_{j} f_{0 l}+\xi_{l} f_{0 j}\right) \\
= & \left(\varepsilon_{\mathrm{r}}\right)_{s l} \xi_{0} \xi_{0} f_{0 l}-\left(\varepsilon_{\mathrm{r}}\right)_{k l} \xi_{k} \xi_{s} f_{0 l}+\epsilon_{s i p}\left(\mu_{\mathrm{r}}^{-1}\right)_{p q} \epsilon_{q j l} \xi_{i} \xi_{j} f_{0 l},
\end{aligned}
$$

\section{VERSÃO 1a:}

$$
\begin{aligned}
& \Lambda_{0 s}^{0 l} \xi_{0} \xi_{0} f_{0 l}-\Lambda_{a s}^{n l} \xi_{a} \xi_{n} f_{0 l}+\Lambda_{a 0}^{0 l} \xi_{a} \xi_{0} f_{s l}+\Lambda_{a 0}^{n 0} \xi_{a} \xi_{n} f_{s 0} \\
&+ \Lambda_{a 0}^{0 l} \xi_{a}\left(\xi_{0} f_{l s}-\xi_{l} f_{0 s}+\xi_{s} f_{0 l}\right) \\
&=\left(\varepsilon_{\mathrm{r}}\right)_{s l} \xi_{0} \xi_{0} f_{0 l}+\epsilon_{s i p}\left(\mu_{\mathrm{r}}^{-1}\right)_{p q} \epsilon_{q j l} \xi_{i} \xi_{j} f_{0 l}+\left(\varepsilon_{\mathrm{r}}\right)_{k l} \xi_{k} \xi_{0} f_{l s}-\left(\varepsilon_{\mathrm{r}}\right)_{k l} \xi_{k} \xi_{l} f_{0 s} \\
& \quad-\left(\varepsilon_{\mathrm{r}}\right)_{k l} \xi_{k}\left(\xi_{0} f_{l s}-\xi_{l} f_{0 s}+\xi_{s} f_{0 l}\right) \\
&=\left(\varepsilon_{\mathrm{r}}\right)_{s l} \xi_{0} \xi_{0} f_{0 l}-\left(\varepsilon_{\mathrm{r}}\right)_{k l} \xi_{k} \xi_{s} f_{0 l}+\epsilon_{s i p}\left(\mu_{\mathrm{r}}^{-1}\right)_{p q} \epsilon_{q j l} \xi_{i} \xi_{j} f_{0 l},
\end{aligned}
$$

\section{VERSÃO 2:}

$$
\begin{aligned}
\frac{1}{2} & \left(\Lambda^{-1}\right)_{s}^{b} \Lambda_{0 b}^{0 l} \xi_{0} \xi_{0} f_{0 l}+\frac{1}{2}\left(\Lambda^{-1}\right)_{s}^{b} \Lambda_{0 b}^{k 0} \xi_{0} \xi_{0} f_{k 0}-\frac{1}{2}\left(\Lambda^{-1}\right)_{s}^{b} \Lambda_{a b}^{k l} \xi_{a} \xi_{0} f_{k l} \\
+ & \frac{1}{2} \Lambda_{a 0}^{0 l} \xi_{a} \xi_{s} f_{0 l}+\frac{1}{2} \Lambda_{a 0}^{k 0} \xi_{a} \xi_{s} f_{k 0} \\
+ & \frac{1}{2}\left(\Lambda^{-1}\right)_{s}^{b} \Lambda_{a b}^{k l} \xi_{a}\left(\xi_{0} f_{k l}-\xi_{k} f_{0 l}+\xi_{l} f_{0 k}\right) \\
= & \xi_{0} \xi_{0} f_{0 s}-\left(\varepsilon_{\mathrm{r}}\right)_{k l} \xi_{k} \xi_{s} f_{0 l}+\frac{1}{2}\left(\varepsilon_{\mathrm{r}}^{-1}\right)_{s t} \epsilon_{t i p}\left(\mu_{\mathrm{r}}^{-1}\right)_{p q} \epsilon_{q j l} \xi_{i} \xi_{0} f_{j l} \\
& \quad-\frac{1}{2}\left(\varepsilon_{\mathrm{r}}^{-1}\right)_{s t} \epsilon_{t i p}\left(\mu_{\mathrm{r}}^{-1}\right)_{p q} \epsilon_{q j l} \xi_{i}\left(\xi_{0} f_{j l}-\xi_{j} f_{0 l}+\xi_{l} f_{0 j}\right) \\
= & \xi_{0} \xi_{0} f_{0 s}-\left(\varepsilon_{\mathrm{r}}\right)_{k l} \xi_{k} \xi_{s} f_{0 l}+\left(\varepsilon_{\mathrm{r}}^{-1}\right)_{s t} \epsilon_{t i p}\left(\mu_{\mathrm{r}}^{-1}\right)_{p q} \epsilon_{q j l} \xi_{i} \xi_{j} f_{0 l},
\end{aligned}
$$


VERSÃO 2 MELHORADA:

$$
\begin{aligned}
\frac{1}{2}( & \left.\Lambda^{-1}\right)_{s}^{b} \Lambda_{0 b}^{0 l} \xi_{0} \xi_{0} f_{0 l}+\frac{1}{2}\left(\Lambda^{-1}\right)_{s}^{b} \Lambda_{0 b}^{k 0} \xi_{0} \xi_{0} f_{k 0}-\frac{1}{2}\left(\Lambda^{-1}\right)_{s}^{b} \Lambda_{a b}^{k l} \xi_{a} \xi_{0} f_{k l} \\
+ & \frac{1}{2} \Lambda_{a 0}^{0 l} \xi_{a} \xi_{s} f_{0 l}+\frac{1}{2} \Lambda_{a 0}^{k 0} \xi_{a} \xi_{s} f_{k 0} \\
+ & \frac{1}{2}\left(\Lambda^{-1}\right)_{s}^{b} \Lambda_{a b}^{k l} \xi_{a}\left(\xi_{0} f_{k l}-\xi_{k} f_{0 l}+\xi_{l} f_{0 k}\right) \\
= & \xi_{0} \xi_{0} f_{0 s}-\left(\varepsilon_{\mathrm{r}}\right)_{k l} \xi_{k} \xi_{s} f_{0 l}+\frac{1}{2}\left(\varepsilon_{\mathrm{r}}^{-1}\right)_{s t} \epsilon_{t i p}\left(\mu_{\mathrm{r}}^{-1}\right)_{p q} \epsilon_{q j l} \xi_{i} \xi_{0} f_{j l} \\
& \quad-\frac{1}{2}\left(\varepsilon_{\mathrm{r}}^{-1}\right)_{s t} \epsilon_{t i p}\left(\mu_{\mathrm{r}}^{-1}\right)_{p q} \epsilon_{q j l} \xi_{i}\left(\xi_{0} f_{j l}-\xi_{j} f_{0 l}+\xi_{l} f_{0 j}\right) \\
= & \xi_{0} \xi_{0} f_{0 s}-\left(\varepsilon_{\mathrm{r}}\right)_{k l} \xi_{k} \xi_{s} f_{0 l}+\left(\varepsilon_{\mathrm{r}}^{-1}\right)_{s t} \epsilon_{t i p}\left(\mu_{\mathrm{r}}^{-1}\right)_{p q} \epsilon_{q j l} \xi_{i} \xi_{j} f_{0 l},
\end{aligned}
$$

\section{VERSÃO 2a:}

$$
\begin{aligned}
&\left(\Lambda^{-1}\right)_{s}^{b} \Lambda_{0 b}^{0 l} \xi_{0} \xi_{0} f_{0 l}-\left(\Lambda^{-1}\right)_{s}^{b} \Lambda_{a b}^{n l} \xi_{a} \xi_{n} f_{0 l}+\Lambda_{a 0}^{0 l} \xi_{a} \xi_{0} f_{s l}+\Lambda_{a 0}^{n 0} \xi_{a} \xi_{n} f_{s 0} \\
&+ \Lambda_{a 0}^{0 l} \xi_{a}\left(\xi_{0} f_{l s}-\xi_{l} f_{0 s}+\xi_{s} f_{0 l}\right) \\
&= \xi_{0} \xi_{0} f_{0 s}+\left(\varepsilon_{\mathrm{r}}^{-1}\right)_{s t} \epsilon_{t i p}\left(\mu_{\mathrm{r}}^{-1}\right)_{p q} \epsilon_{q j l} \xi_{i} \xi_{j} f_{0 l}+\left(\varepsilon_{\mathrm{r}}\right)_{k l} \xi_{k} \xi_{0} f_{l s}-\left(\varepsilon_{\mathrm{r}}\right)_{k l} \xi_{k} \xi_{l} f_{0 s} \\
& \quad-\left(\varepsilon_{\mathrm{r}}\right)_{k l} \xi_{k}\left(\xi_{0} f_{l s}-\xi_{l} f_{0 s}+\xi_{s} f_{0 l}\right) \\
&= \xi_{0} \xi_{0} f_{0 s}-\left(\varepsilon_{\mathrm{r}}\right)_{k l} \xi_{k} \xi_{s} f_{0 l}+\left(\varepsilon_{\mathrm{r}}^{-1}\right)_{s t} \epsilon_{t i p}\left(\mu_{\mathrm{r}}^{-1}\right)_{p q} \epsilon_{q j l} \xi_{i} \xi_{j} f_{0 l},
\end{aligned}
$$

VERSÃO 2b:

$$
\begin{aligned}
& \frac{1}{4}\left(\Lambda^{-1}\right)_{s}^{b} \Lambda_{0 b}^{0 l} \xi_{0} \xi_{0} f_{0 l}+\frac{1}{4}\left(\Lambda^{-1}\right)_{s}^{b} \Lambda_{0 b}^{k 0} \xi_{0} \xi_{0} f_{k 0}-\frac{1}{4}\left(\Lambda^{-1}\right)_{s}^{b} \Lambda_{a b}^{k l} \xi_{a} \xi_{0} f_{k l} \\
&+\frac{1}{4} \Lambda_{a 0}^{0 l} \xi_{a} \xi_{s} f_{0 l}+\frac{1}{4} \Lambda_{a 0}^{k 0} \xi_{a} \xi_{s} f_{k 0} \\
&+\frac{1}{2}\left(\Lambda^{-1}\right)_{s}^{b} \Lambda_{0 b}^{0 l} \xi_{0} \xi_{0} f_{0 l}-\frac{1}{2}\left(\Lambda^{-1}\right)_{s}^{b} \Lambda_{a b}^{n l} \xi_{a} \xi_{n} f_{0 l} \\
&+\frac{1}{2} \Lambda_{a 0}^{0 l} \xi_{a} \xi_{0} f_{s l}+\frac{1}{2} \Lambda_{a 0}^{n 0} \xi_{a} \xi_{n} f_{s 0} \\
&+ \frac{1}{2} \Lambda_{a 0}^{0 l} \xi_{a}\left(\xi_{s} f_{0 l}-\xi_{l} f_{0 s}-\xi_{0} f_{s l}\right) \\
&- \frac{1}{4}\left(\Lambda^{-1}\right)_{s}^{b} \Lambda_{a b}^{k l} \xi_{a}\left(\xi_{k} f_{0 l}-\xi_{l} f_{0 k}-\xi_{0} f_{k l}\right) \\
&= \xi_{0} \xi_{0} f_{0 s}-\frac{1}{2}\left(\varepsilon_{\mathrm{r}}\right)_{k l} \xi_{k} \xi_{s} f_{0 l}-\frac{1}{2}\left(\varepsilon_{\mathrm{r}}\right)_{k l} \xi_{k} \xi_{l} f_{0 s}-\frac{1}{2}\left(\varepsilon_{\mathrm{r}}\right)_{k l} \xi_{k} \xi_{0} f_{s l} \\
& \quad+\frac{1}{4}\left(\varepsilon_{\mathrm{r}}^{-1}\right)_{s t} \epsilon_{t i p}\left(\mu_{\mathrm{r}}^{-1}\right)_{p q} \epsilon_{q k l} \xi_{i}\left(\xi_{k} f_{0 l}-\xi_{l} f_{0 k}+\xi_{0} f_{k l}\right) \\
& \quad-\frac{1}{2}\left(\varepsilon_{\mathrm{r}}\right)_{k l} \xi_{k}\left(\xi_{s} f_{0 l}-\xi_{l} f_{0 s}-\xi_{0} f_{s l}\right) \\
&+\frac{1}{4}\left(\varepsilon_{\mathrm{r}}^{-1}\right)_{s t} \epsilon_{t i p}\left(\mu_{\mathrm{r}}^{-1}\right)_{p q} \epsilon_{q k l} \xi_{i}\left(\xi_{k} f_{0 l}-\xi_{l} f_{0 k}-\xi_{0} f_{k l}\right) \\
&= \xi_{0} \xi_{0} f_{0 s}-\left(\varepsilon_{\mathrm{r}}\right)_{k l} \xi_{k} \xi_{s} f_{0 l}+\left(\varepsilon_{\mathrm{r}}^{-1}\right)_{s t} \epsilon_{t i p}\left(\mu_{\mathrm{r}}^{-1}\right)_{p q} \epsilon_{q k l} \xi_{i} \xi_{k} f_{0 l},
\end{aligned}
$$

enquanto que no caso $\rho=r$ e $\sigma=s$, ela vale 


\section{VERSÃO 1:}

$$
\begin{aligned}
& \frac{1}{2} \Lambda_{0 s}^{0 l} \xi_{0} \xi_{r} f_{0 l}+\frac{1}{2} \Lambda_{0 s}^{k 0} \xi_{0} \xi_{r} f_{k 0}-\frac{1}{2} \Lambda_{a s}^{k l} \xi_{a} \xi_{r} f_{k l} \\
&-\frac{1}{2} \Lambda_{0 r}^{0 l} \xi_{0} \xi_{s} f_{0 l}-\frac{1}{2} \Lambda_{0 r}^{k 0} \xi_{0} \xi_{s} f_{k 0}+\frac{1}{2} \Lambda_{a r}^{k l} \xi_{a} \xi_{s} f_{k l} \\
&- \frac{1}{2} \Lambda_{0 s}^{0 l} \xi_{0}\left(\xi_{r} f_{0 l}-\xi_{l} f_{0 r}-\xi_{0} f_{r l}\right) \\
&+ \frac{\frac{1}{2} \Lambda_{0 r}^{0 l} \xi_{0}\left(\xi_{s} f_{0 l}-\xi_{l} f_{0 s}+\xi_{0} f_{l s}\right)}{=} \\
&\left(\varepsilon_{\mathrm{r}}\right)_{s l} \xi_{0} \xi_{r} f_{0 l}-\frac{1}{2} \epsilon_{s i p}\left(\mu_{\mathrm{r}}^{-1}\right)_{p q} \epsilon_{q k l} \xi_{i} \xi_{r} f_{k l} \\
& \quad-\left(\varepsilon_{\mathrm{r}}\right)_{r l} \xi_{0} \xi_{s} f_{0 l}+\frac{1}{2} \epsilon_{r i p}\left(\mu_{\mathrm{r}}^{-1}\right)_{p q} \epsilon_{q k l} \xi_{i} \xi_{s} f_{k l} \\
& \quad-\frac{1}{2}\left(\varepsilon_{\mathrm{r}}\right)_{s l} \xi_{0}\left(\xi_{r} f_{0 l}-\xi_{l} f_{0 r}-\xi_{0} f_{r l}\right) \\
& \quad+\frac{1}{2}\left(\varepsilon_{\mathrm{r}}\right)_{r l} \xi_{0}\left(\xi_{s} f_{0 l}-\xi_{l} f_{0 s}+\xi_{0} f_{l s}\right) \\
&= \text { não parece ser útil },
\end{aligned}
$$

\section{VERSÃO 1a:}

$$
\begin{aligned}
\Lambda_{0 s}^{0 l} \xi_{0} \xi_{0} f_{r l}+\Lambda_{0 s}^{n 0} \xi_{0} \xi_{n} f_{r 0}-\Lambda_{a s}^{n l} \xi_{a} \xi_{n} f_{r l} \\
-\Lambda_{0 r}^{0 l} \xi_{0} \xi_{0} f_{s l}+\Lambda_{0 r}^{n 0} \xi_{0} \xi_{n} f_{s 0}-\Lambda_{a r}^{n l} \xi_{a} \xi_{n} f_{s l} \\
-\Lambda_{0 s}^{n 0} \xi_{0}\left(\xi_{n} f_{r 0}+\xi_{r} f_{0 n}-\xi_{0} f_{r n}\right) \\
+\Lambda_{0 r}^{n 0} \xi_{0}\left(\xi_{n} f_{s 0}+\xi_{s} f_{0 n}+\xi_{0} f_{n s}\right) \\
=\left(\varepsilon_{\mathrm{r}}\right)_{s l} \xi_{0} \xi_{0} f_{r l}-\left(\varepsilon_{\mathrm{r}}\right)_{s l} \xi_{0} \xi_{l} f_{r 0}+\epsilon_{s i p}\left(\mu_{\mathrm{r}}^{-1}\right)_{p q} \epsilon_{q k l} \xi_{i} \xi_{k} f_{r l} \\
\quad-\left(\varepsilon_{\mathrm{r}}\right)_{r l} \xi_{0} \xi_{0} f_{s l}+\left(\varepsilon_{\mathrm{r}}\right)_{r l} \xi_{0} \xi_{l} f_{s 0}-\epsilon_{r i p}\left(\mu_{\mathrm{r}}^{-1}\right)_{p q} \epsilon_{q k l} \xi_{i} \xi_{k} f_{s l} \\
\quad+\left(\varepsilon_{\mathrm{r}}\right)_{s l} \xi_{0}\left(\xi_{l} f_{r 0}-\xi_{r} f_{l 0}-\xi_{0} f_{r l}\right) \\
\quad-\left(\varepsilon_{\mathrm{r}}\right)_{r l} \xi_{0}\left(\xi_{l} f_{s 0}-\xi_{s} f_{l 0}-\xi_{0} f_{s l}\right)
\end{aligned}
$$

$=$ não parece ser útil , 


\section{VERSÃO 2:}

$$
\begin{aligned}
& \frac{1}{2}\left(\Lambda^{-1}\right)_{s}^{b} \Lambda_{0 b}^{0 l} \xi_{0} \xi_{r} f_{0 l}+\frac{1}{2}\left(\Lambda^{-1}\right)_{s}^{b} \Lambda_{0 b}^{k 0} \xi_{0} \xi_{r} f_{k 0}-\frac{1}{2}\left(\Lambda^{-1}\right)_{s}^{b} \Lambda_{a b}^{k l} \xi_{a} \xi_{r} f_{k l} \\
& -\frac{1}{2}\left(\Lambda^{-1}\right)_{r}^{b} \Lambda_{0 b}^{0 l} \xi_{0} \xi_{s} f_{0 l}-\frac{1}{2}\left(\Lambda^{-1}\right)_{r}^{b} \Lambda_{0 b}^{k 0} \xi_{0} \xi_{s} f_{k 0}+\frac{1}{2}\left(\Lambda^{-1}\right)_{r}^{b} \Lambda_{a b}^{k l} \xi_{a} \xi_{s} f_{k l} \\
& -\frac{1}{2}\left(\Lambda^{-1}\right)_{s}^{b} \Lambda_{0 b}^{0 l} \xi_{0}\left(\xi_{r} f_{0 l}-\xi_{l} f_{0 r}-\xi_{0} f_{r l}\right) \\
& +\frac{1}{2}\left(\Lambda^{-1}\right)_{r}^{b} \Lambda_{0 b}^{0 l} \xi_{0}\left(\xi_{s} f_{0 l}-\xi_{l} f_{0 s}+\xi_{0} f_{l s}\right) \\
& =\xi_{0} \xi_{r} f_{0 s}+\frac{1}{2}\left(\varepsilon_{\mathrm{r}}^{-1}\right)_{s t} \epsilon_{t i p}\left(\mu_{\mathrm{r}}^{-1}\right)_{p q} \epsilon_{q k l} \xi_{i} \xi_{r} f_{k l} \\
& \quad-\xi_{0} \xi_{s} f_{0 r}-\frac{1}{2}\left(\varepsilon_{\mathrm{r}}^{-1}\right)_{r t} \epsilon_{t i p}\left(\mu_{\mathrm{r}}^{-1}\right)_{p q} \epsilon_{q k l} \xi_{i} \xi_{s} f_{k l} \\
& \quad-\xi_{0}\left(\xi_{r} f_{0 s}-\xi_{s} f_{0 r}-\xi_{0} f_{r s}\right) \\
& =\xi_{0} \xi_{0} f_{r s}+\frac{1}{2}\left(\varepsilon_{\mathrm{r}}^{-1}\right)_{s t} \epsilon_{t i p}\left(\mu_{\mathrm{r}}^{-1}\right)_{p q} \epsilon_{q k l} \xi_{i} \xi_{r} f_{k l} \\
& -\frac{1}{2}\left(\varepsilon_{\mathrm{r}}^{-1}\right)_{r t} \epsilon_{t i p}\left(\mu_{\mathrm{r}}^{-1}\right)_{p q} \epsilon_{q k l} \xi_{i} \xi_{s} f_{k l} .
\end{aligned}
$$

\section{VERSÃO 2 MELHORADA:}

$$
\begin{aligned}
\frac{1}{2} & \left(\Lambda^{-1}\right)_{s}^{b} \Lambda_{0 b}^{0 l} \xi_{0} \xi_{r} f_{0 l}+\frac{1}{2}\left(\Lambda^{-1}\right)_{s}^{b} \Lambda_{0 b}^{k 0} \xi_{0} \xi_{r} f_{k 0}-\frac{1}{2}\left(\Lambda^{-1}\right)_{s}^{b} \Lambda_{i b}^{k l} \xi_{i} \xi_{r} f_{k l} \\
- & \frac{1}{2}\left(\Lambda^{-1}\right)_{r}^{b} \Lambda_{0 b}^{0 l} \xi_{0} \xi_{s} f_{0 l}-\frac{1}{2}\left(\Lambda^{-1}\right)_{r}^{b} \Lambda_{0 b}^{k 0} \xi_{0} \xi_{s} f_{k 0}+\frac{1}{2}\left(\Lambda^{-1}\right)_{r}^{b} \Lambda_{i b}^{k l} \xi_{i} \xi_{s} f_{k l} \\
- & \frac{1}{4}\left(\Lambda^{-1}\right)_{s}^{b} \Lambda_{0 b}^{0 l} \xi_{0}\left(\xi_{r} f_{0 l}-\xi_{0} f_{r l}+\xi_{l} f_{r 0}\right) \\
- & \frac{1}{4}\left(\Lambda^{-1}\right)_{s}^{b} \Lambda_{0 b}^{k 0} \xi_{0}\left(\xi_{r} f_{k 0}-\xi_{k} f_{r 0}+\xi_{0} f_{r k}\right) \\
+ & \frac{1}{4}\left(\Lambda^{-1}\right)_{s}^{b} \Lambda_{i b}^{k l} \xi_{i}\left(\xi_{r} f_{k l}-\xi_{k} f_{r l}+\xi_{l} f_{r k}\right) \\
+ & \frac{1}{4}\left(\Lambda^{-1}\right)_{r}^{b} \Lambda_{0 b}^{0 l} \xi_{0}\left(\xi_{s} f_{0 l}-\xi_{0} f_{s l}+\xi_{l} f_{s 0}\right) \\
+ & \frac{1}{4}\left(\Lambda^{-1}\right)_{r}^{b} \Lambda_{0 b}^{k 0} \xi_{0}\left(\xi_{s} f_{k 0}-\xi_{k} f_{s 0}+\xi_{0} f_{s k}\right) \\
- & \frac{1}{4}\left(\Lambda^{-1}\right)_{r}^{b} \Lambda_{i b}^{k l} \xi_{i}\left(\xi_{s} f_{k l}-\xi_{k} f_{s l}+\xi_{l} f_{s k}\right) \\
= & \xi_{0} \xi_{r} f_{0 s}+\frac{1}{2}\left(\varepsilon_{\mathrm{r}}^{-1}\right)_{s t} \epsilon_{t i p}\left(\mu_{\mathrm{r}}^{-1}\right)_{p q} \epsilon_{q k l} \xi_{i} \xi_{r} f_{k l} \\
& -\xi_{0} \xi_{s} f_{0 r}-\frac{1}{2}\left(\varepsilon_{\mathrm{r}}^{-1}\right)_{r t} \epsilon_{t i p}\left(\mu_{\mathrm{r}}^{-1}\right)_{p q} \epsilon_{q k l} \xi_{i} \xi_{s} f_{k l} \\
& -\frac{1}{2} \xi_{0}\left(\xi_{r} f_{0 s}-\xi_{s} f_{0 r}-\xi_{0} f_{r s}\right) \\
& +\frac{1}{2} \xi_{0}\left(\xi_{s} f_{0 r}-\xi_{r} f_{0 s}-\xi_{0} f_{s r}\right) \\
& -\frac{1}{4}\left(\varepsilon_{\mathrm{r}}^{-1}\right)_{s t} \epsilon_{t i p}\left(\mu_{\mathrm{r}}^{-1}\right)_{p q} \epsilon_{q k l} \xi_{i}\left(\xi_{r} f_{k l}-\xi_{k} f_{r l}+\xi_{l} f_{r k}\right) \\
& +\frac{1}{4}\left(\varepsilon_{\mathrm{r}}^{-1}\right)_{r t} \epsilon_{t i p}\left(\mu_{\mathrm{r}}^{-1}\right)_{p q} \epsilon_{q k l} \xi_{i}\left(\xi_{s} f_{k l}-\xi_{k} f_{s l}+\xi_{l} f_{s k}\right) \\
= & \xi_{0} \xi_{0} f_{r s}+\frac{1}{4}\left(\varepsilon_{\mathrm{r}}^{-1}\right)_{s t} \epsilon_{t i p}\left(\mu_{\mathrm{r}}^{-1}\right)_{p q} \epsilon_{q k l} \xi_{i}\left(\xi_{r} f_{k l}+\xi_{k} f_{r l}-\xi_{l} f_{r k}\right) \\
& -\frac{1}{4}\left(\varepsilon_{\mathrm{r}}^{-1}\right)_{r t} \epsilon_{t i p}\left(\mu_{\mathrm{r}}^{-1}\right)_{p q} \epsilon_{q k l} \xi_{i}\left(\xi_{s} f_{k l}+\xi_{k} f_{s l}-\xi_{l} f_{s k}\right) .
\end{aligned}
$$




\section{VERSÃO 2a:}

$$
\begin{aligned}
& \left(\Lambda^{-1}\right)_{s}^{b} \Lambda_{0 b}^{0 l} \xi_{0} \xi_{0} f_{r l}+\left(\Lambda^{-1}\right)_{s}^{b} \Lambda_{0 b}^{n 0} \xi_{0} \xi_{n} f_{r 0}-\left(\Lambda^{-1}\right)_{s}^{b} \Lambda_{a b}^{n l} \xi_{a} \xi_{n} f_{r l} \\
& -\left(\Lambda^{-1}\right)_{r}^{b} \Lambda_{0 b}^{0 l} \xi_{0} \xi_{0} f_{s l}-\left(\Lambda^{-1}\right)_{r}^{b} \Lambda_{0 b}^{n 0} \xi_{0} \xi_{n} f_{s 0}+\left(\Lambda^{-1}\right)_{r}^{b} \Lambda_{a b}^{n l} \xi_{a} \xi_{n} f_{s l} \\
& -\quad \frac{1}{2}\left(\Lambda^{-1}\right)_{s}^{b} \Lambda_{0 b}^{0 l} \xi_{0}\left(\xi_{l} f_{0 r}-\xi_{r} f_{0 l}+\xi_{0} f_{r l}\right) \\
& + \\
& +\frac{1}{2}\left(\Lambda^{-1}\right)_{r}^{b} \Lambda_{0 b}^{0 l} \xi_{0}\left(\xi_{l} f_{0 s}-\xi_{s} f_{0 l}-\xi_{0} f_{l s}\right) \\
& =\xi_{0} \xi_{0} f_{r s}+\xi_{0} \xi_{s} f_{0 r}+\left(\varepsilon_{\mathrm{r}}^{-1}\right)_{s t} \epsilon_{t i p}\left(\mu_{\mathrm{r}}^{-1}\right)_{p q} \epsilon_{q k l} \xi_{i} \xi_{k} f_{r l} \\
& \quad-\xi_{0} \xi_{0} f_{s r}-\xi_{0} \xi_{r} f_{0 s}-\left(\varepsilon_{\mathrm{r}}^{-1}\right)_{r t} \epsilon_{t i p}\left(\mu_{\mathrm{r}}^{-1}\right)_{p q} \epsilon_{q k l} \xi_{i} \xi_{k} f_{s l} \\
& \quad+\xi_{0}\left(\xi_{r} f_{0 s}-\xi_{s} f_{0 r}-\xi_{0} f_{r s}\right) \\
& =\xi_{0} \xi_{0} f_{r s}+\left(\varepsilon_{\mathrm{r}}^{-1}\right)_{s t} \epsilon_{t i p}\left(\mu_{\mathrm{r}}^{-1}\right)_{p q} \epsilon_{q k l} \xi_{i} \xi_{k} f_{r l} \\
& -\left(\varepsilon_{\mathrm{r}}^{-1}\right)_{r t} \epsilon_{t i p}\left(\mu_{\mathrm{r}}^{-1}\right)_{p q} \epsilon_{q k l} \xi_{i} \xi_{k} f_{s l} .
\end{aligned}
$$

VERSÃO 2b:

$$
\begin{aligned}
& \frac{1}{4}\left(\Lambda^{-1}\right)_{s}^{b} \Lambda_{0 b}^{0 l} \xi_{0} \xi_{r} f_{0 l}+\frac{1}{4}\left(\Lambda^{-1}\right)_{s}^{b} \Lambda_{0 b}^{k 0} \xi_{0} \xi_{r} f_{k 0}-\frac{1}{4}\left(\Lambda^{-1}\right)_{s}^{b} \Lambda_{a b}^{k l} \xi_{a} \xi_{r} f_{k l} \\
&-\frac{1}{4}\left(\Lambda^{-1}\right)_{r}^{b} \Lambda_{0 b}^{0 l} \xi_{0} \xi_{s} f_{0 l}-\frac{1}{4}\left(\Lambda^{-1}\right)_{r}^{b} \Lambda_{0 b}^{k 0} \xi_{0} \xi_{s} f_{k 0}+\frac{1}{4}\left(\Lambda^{-1}\right)_{r}^{b} \Lambda_{a b}^{k l} \xi_{a} \xi_{s} f_{k l} \\
&+\frac{1}{2}\left(\Lambda^{-1}\right)_{s}^{b} \Lambda_{0 b}^{0 l} \xi_{0} \xi_{0} f_{r l}+\frac{1}{2}\left(\Lambda^{-1}\right)_{s}^{b} \Lambda_{0 b}^{n 0} \xi_{0} \xi_{n} f_{r 0}-\frac{1}{2}\left(\Lambda^{-1}\right)_{s}^{b} \Lambda_{a b}^{n l} \xi_{a} \xi_{n} f_{r l} \\
&-\frac{1}{2}\left(\Lambda^{-1}\right)_{r}^{b} \Lambda_{0 b}^{0 l} \xi_{0} \xi_{0} f_{s l}-\frac{1}{2}\left(\Lambda^{-1}\right)_{r}^{b} \Lambda_{0 b}^{n 0} \xi_{0} \xi_{n} f_{s 0}+\frac{1}{2}\left(\Lambda^{-1}\right)_{r}^{b} \Lambda_{a b}^{n l} \xi_{a} \xi_{n} f_{s l} \\
&+ \frac{1}{4}\left(\Lambda^{-1}\right)_{s}^{b} \Lambda_{a b}^{k l} \xi_{a}\left(\xi_{k} f_{r l}-\xi_{l} f_{r k}-\xi_{r} f_{k l}\right) \\
&- \frac{1}{4}\left(\Lambda^{-1}\right)_{r}^{b} \Lambda_{a b}^{k l} \xi_{a}\left(\xi_{k} f_{s l}-\xi_{l} f_{s k}-\xi_{s} f_{k l}\right) \\
&= \frac{1}{2} \xi_{0} \xi_{r} f_{0 s}+\frac{1}{4}\left(\varepsilon_{\mathrm{r}}^{-1}\right)_{s t} \epsilon_{t i p}\left(\mu_{\mathrm{r}}^{-1}\right)_{p q} \epsilon_{q k l} \xi_{i} \xi_{r} f_{k l} \\
&-\frac{1}{2} \xi_{0} \xi_{s} f_{0 r}-\frac{1}{4}\left(\varepsilon_{\mathrm{r}}^{-1}\right)_{r t} \epsilon_{t i p}\left(\mu_{\mathrm{r}}^{-1}\right)_{p q} \epsilon_{q k l} \xi_{i} \xi_{s} f_{k l} \\
&+\frac{1}{2} \xi_{0} \xi_{0} f_{r s}+\frac{1}{2} \xi_{0} \xi_{s} f_{0 r}+\frac{1}{2}\left(\varepsilon_{\mathrm{r}}^{-1}\right)_{s t} \epsilon_{t i p}\left(\mu_{\mathrm{r}}^{-1}\right)_{p q} \epsilon_{q k l} \xi_{i} \xi_{k} f_{r l} \\
&-\frac{1}{2} \xi_{0} \xi_{0} f_{s r}-\frac{1}{2} \xi_{0} \xi_{r} f_{0 s}-\frac{1}{2}\left(\varepsilon_{\mathrm{r}}^{-1}\right)_{r t} \frac{1}{2} \epsilon_{t i p}\left(\mu_{\mathrm{r}}^{-1}\right)_{p q} \epsilon_{q k l} \xi_{i} \xi_{k} f_{s l} \\
&-\frac{1}{4}\left(\varepsilon_{\mathrm{r}}^{-1}\right)_{s t} \epsilon_{t i p}\left(\mu_{\mathrm{r}}^{-1}\right)_{p q} \epsilon_{q k l} \xi_{i}\left(\xi_{k} f_{r l}-\xi_{l} f_{r k}-\xi_{r} f_{k l}\right) \\
&+\frac{1}{4}\left(\varepsilon_{\mathrm{r}}^{-1}\right)_{r t} \epsilon_{t i p}\left(\mu_{\mathrm{r}}^{-1}\right)_{p q} \epsilon_{q k l} \xi_{i}\left(\xi_{k} f_{s l}-\xi_{l} f_{s k}-\xi_{s} f_{k l}\right) \\
&= \xi_{0} \xi_{0} f_{r s}+\frac{1}{2}\left(\varepsilon_{\mathrm{r}}^{-1}\right)_{s t} \epsilon_{t i p}\left(\mu_{\mathrm{r}}^{-1}\right)_{p q} \epsilon_{q k l} \xi_{i} \xi_{r} f_{k l} \\
& \quad-\frac{1}{2}\left(\varepsilon_{\mathrm{r}}^{-1}\right)_{r t} \epsilon_{t i p}\left(\mu_{\mathrm{r}}^{-1}\right)_{p q} \epsilon_{q k l} \xi_{i} \xi_{s} f_{k l} .
\end{aligned}
$$

Com estas inclusões, fica claro que as componentes elétricas $\left(F_{0 s}\right)$ e as componentes magnéticas $\left(F_{r s}\right)$ da equação (??) reproduzem, respectivamente, as equações (3.20) e (3.21). 
O que ainda falta é encontrar uma forma covariante para os termos exibidas nas caixas acima, que valem 0 devido à equação homogênea, para que possamos incluí-los na equação (??). Isso levará a uma modificação substancial do resultado final para o símbolo principal do operador que rege a equação de onda correta para o tensor eletromagnético $F$.

Verifiquemos explicitamente que estas equações coincidem com as que foram deduzidas na Seção 7.2.

Para tanto, notamos que, quando $\rho=0$ e $\sigma=s$,

$$
\begin{aligned}
& \left(\sigma_{F}^{\mu \nu}\right)_{0 s}^{\kappa \lambda} \partial_{\mu} \partial_{\nu} F_{\kappa \lambda}=\frac{1}{2} \eta^{\mu \alpha}\left(\Lambda^{-1}\right)_{s}^{b} \Lambda_{\alpha b}^{\kappa \lambda} \partial_{\mu} \partial_{0} F_{\kappa \lambda}-\frac{1}{2} \eta^{\mu \alpha} \Lambda_{\alpha 0}^{\kappa \lambda} \partial_{\mu} \partial_{s} F_{\kappa \lambda} \\
& \quad=\left(\Lambda^{-1}\right)_{s}^{b} \Lambda_{0 b}^{0 l} \partial_{0} \partial_{0} F_{0 l}-\frac{1}{2}\left(\Lambda^{-1}\right)_{s}^{b} \Lambda_{j b}^{k l} \partial_{j} \partial_{0} F_{k l}+\Lambda_{j 0}^{k 0} \partial_{j} \partial_{s} F_{k 0} \\
& =\partial_{0} \partial_{0} F_{0 s}-\partial_{s}\left(\Lambda_{j 0}^{k 0} \partial_{j} F_{0 k}\right)+\frac{1}{2}\left(\Lambda^{-1}\right)_{s}^{b} \Lambda_{j b}^{k l} \partial_{j}\left(\partial_{k} F_{l 0}+\partial_{l} F_{0 k}\right) \\
& =\partial_{0} \partial_{0} F_{0 s}-\partial_{s}\left(\left(\varepsilon_{\mathrm{r}}\right)_{j k} \partial_{j} F_{0 k}\right)+\left(\varepsilon_{\mathrm{r}}^{-1}\right)_{s t} \epsilon_{t i p}\left(\mu_{\mathrm{r}}^{-1}\right)_{p q} \epsilon_{q k l} \partial_{i} \partial_{k} F_{0 l} \\
& \quad=\frac{1}{\varkappa c}\left(\frac{1}{c^{2}} \frac{\partial^{2} E_{s}}{\partial t^{2}}-\partial_{s}\left(\left(\varepsilon_{\mathrm{r}}\right)_{j k} \partial_{j} E_{k}\right)+\left(\varepsilon_{\mathrm{r}}^{-1}\right)_{s t} \epsilon_{t i p} \partial_{i}\left(\left(\mu_{\mathrm{r}}^{-1}\right)_{p q} \epsilon_{q k l} \partial_{k} E_{l}\right)\right) \\
& =\frac{1}{\varkappa c}\left(\frac{1}{c^{2}} \frac{\partial^{2} \boldsymbol{E}}{\partial t^{2}}-\nabla(\nabla \cdot \boldsymbol{E})+\varepsilon_{\mathrm{r}}^{-1} \nabla \times\left(\mu_{\mathrm{r}}^{-1} \nabla \times \boldsymbol{E}\right)\right)_{s} \\
& =\frac{1}{\varkappa \varepsilon_{0} c}\left(\varepsilon_{\mathrm{r}}^{-1}\right)_{s t}\left(\frac{1}{c^{2}} \frac{\partial^{2} \boldsymbol{D}}{\partial t^{2}}-\varepsilon_{\mathrm{r}} \boldsymbol{\nabla}(\boldsymbol{\nabla} \cdot \boldsymbol{D})+\nabla \times\left(\mu_{\mathrm{r}}^{-1} \nabla \times\left(\varepsilon_{\mathrm{r}}^{-1} \boldsymbol{D}\right)\right)\right)_{t},
\end{aligned}
$$

enquanto que

$$
\varkappa \mu_{0}\left(\partial_{0}\left(\Lambda^{-1} j_{\text {ext }}\right)_{s}-\partial_{s}\left(\Lambda^{-1} j_{\text {ext }}\right)_{0}\right)=-\varkappa \mu_{0}\left(\frac{1}{c} \frac{\partial}{\partial t}\left(\varepsilon_{\mathrm{r}}^{-1} j_{\text {ext }}\right)+c \nabla \rho_{\text {ext }}\right)_{s}
$$

de modo que as componentes elétricas da equação (??) reproduzem as equações $(3.20)$ e $(3.22)(\operatorname{com} \lambda=1)$. 
De forma semelhante, quando $\rho=r$ e $\sigma=s$,

$$
\begin{aligned}
& \frac{1}{2} \epsilon_{k r s}\left(\sigma_{F}^{\mu \nu}\right)_{r s}^{\kappa \lambda} \partial_{\mu} \partial_{\nu} F_{\kappa \lambda}=\frac{1}{2} \epsilon_{k r s} \eta^{\mu \alpha}\left(\Lambda^{-1}\right)_{s}^{b} \Lambda_{\alpha b}^{\kappa \lambda} \partial_{\mu} \partial_{r} F_{\kappa \lambda} \\
& =\epsilon_{k r s}\left(\Lambda^{-1}\right)_{s}^{b} \Lambda_{0 b}^{0 l} \partial_{0} \partial_{r} F_{0 l}-\frac{1}{2} \epsilon_{k r s}\left(\Lambda^{-1}\right)_{s}^{b} \Lambda_{j b}^{k l} \partial_{j} \partial_{r} F_{k l} \\
& =\epsilon_{k r s} \partial_{0} \partial_{r} F_{0 s}+\frac{1}{2} \epsilon_{k r s}\left(\varepsilon_{\mathrm{r}}^{-1}\right)_{s t} \epsilon_{t i p}\left(\mu_{\mathrm{r}}^{-1}\right)_{p q} \epsilon_{q k l} \partial_{i} \partial_{r} F_{k l} \\
& =\frac{1}{2} \epsilon_{k r s} \partial_{0} \partial_{0} F_{r s}+\frac{1}{2} \epsilon_{k r s} \partial_{r}\left(\left(\varepsilon_{\mathrm{r}}^{-1}\right)_{s t} \epsilon_{t i p} \partial_{i}\left(\left(\mu_{\mathrm{r}}^{-1}\right)_{p q} \epsilon_{q k l} F_{k l}\right)\right) \\
& =-\left(\frac{1}{c^{2}} \frac{\partial^{2} B_{k}}{\partial t^{2}}+\epsilon_{k r s} \partial_{r}\left(\left(\varepsilon_{\mathrm{r}}^{-1}\right)_{s t} \epsilon_{t i p} \partial_{i}\left(\left(\mu_{\mathrm{r}}^{-1}\right)_{p q} B_{q}\right)\right)\right) \\
& =-\left(\frac{1}{c^{2}} \frac{\partial^{2} \boldsymbol{B}}{\partial t^{2}}+\nabla \times\left(\varepsilon_{\mathrm{r}}^{-1} \nabla \times\left(\mu_{\mathrm{r}}^{-1} \boldsymbol{B}\right)\right)\right)_{k} \\
& \left.=-\mu_{0}\left(\mu_{\mathrm{r}}\right)_{k l}\left(\frac{1}{c^{2}} \frac{\partial^{2} \boldsymbol{H}}{\partial t^{2}}+\mu_{\mathrm{r}}^{-1} \nabla \times\left(\varepsilon_{\mathrm{r}}^{-1} \nabla \times \boldsymbol{H}\right)\right)\right)_{l},
\end{aligned}
$$

enquanto que

$$
\frac{1}{2} \epsilon_{k r s} \varkappa \mu_{0}\left(\partial_{r}\left(\Lambda^{-1} j_{\text {ext }}\right)_{s}-\partial_{s}\left(\Lambda^{-1} j_{\text {ext }}\right)_{r}\right)=-\varkappa \mu_{0}\left(\nabla \times\left(\varepsilon_{\mathrm{r}}^{-1} j_{\text {ext }}\right)\right)_{k},
$$

de modo que as componentes magnéticas da equação (??) reproduzem as equações $(3.23)$ e $(3.21)(\operatorname{com} \lambda=0)$. 


\section{Bibliografia}

[1] C. Bär, N. Ginoux \& F. Pfäffle. Wave Equations on Lorentzian Manifolds and Quantization, European Mathematical Society 2007.

[2] Birefringence, Wikipedia, https://en.wikipedia.org/wiki/Birefringence

[3] M. Born \& E. Wolf. Principles of Optics, Cambridge University Press 2000.

[4] Elliptic Operator, Encyclopedia of Mathematics, Wikipedia http://encyclopediaofmath.org/wiki/Elliptic_operator

[5] R.P. Feynman, R.B. Leighton \& M. Sands. Lectures on Physics, Vol. II, Basic Books 2011.

[6] M. Forger \& H. Römer. Elementare Feldtheorie, VCH-Wiley, Weinheim 1993.

[7] K.O. Friedrichs. Symmetric hyperbolic linear differential equations, Commun. Pure Appl. Math. 7 (1954) 345-392.

[8] L. Gårding. Linear hyperbolic partial differential equations with constant coefficients, Acta Math. 85 (1951) 1-62.

[9] L. Gårding. Hyperbolic equations in the twentieth century, Sém. Congr. Soc. Math. France 3 (1998) 37-68.

[10] W.H. Greub. Linear Algebra, Springer 1967.

[11] D.J. Griffiths. Introduction to Electrodynamics, Prentice Hall 1999.

[12] J.D. Jackson. Classical Electrodynamics, Wiley 1975.

[13] L.D. Landau \& E.M. Lifshits. Electrodynamics of Continuous Media, Butterworth-Heinemann 1984. 
[14] P.D. Lax. Asymptotic solutions of oscillatory initial value problems, Duke Math. J. 24 (1957) 627-646.

[15] S. Mizohata. Some remarks on the Cauchy problem, J. Math. Kyoto Univ. 1 (1961) 109-127.

[16] J.R. Reitz, F.J. Milford \& R.W. Christy. Foundations of Electromagnetic Theory, Addison-Wesley 1993.

[17] H. Römer. Optics: An Introduction, Wiley 2006.

[18] S.J. Vidal. Operadores diferenciais globalmente hiperbólicos, Tese de Doutorado, IME-USP 2013. 